OPTICAL PROPERTIES

OF

HUMAN BREAST TISSUES 


\title{
OPTICAL PROPERTIES \\ OE NORMAL AND DISEASED HUMAN BREAST TISSUES \\ IN THE VISIBLE AND NEAR INERARED
}

By

VICTOR GEORGE PETERS

\author{
A Project \\ Submitted to the School of Graduate Studies \\ in Partial Fulfilment of the Requirements \\ for the Degree \\ Master of Science \\ (Health and Radiation Physics)
}

McMaster University

August 1989 
Master of Science (1989)

(Health and Radiation Physics)
MCMASTER UNIVERSITY

Hamilton, Ontario

TITLE: Optical Properties of Normal and Diseased Human Breast $T$ issues in the Visible and Near Infrared

AUTHOR: Victor George Peters, B.Sc. (McMaster University)

SUPERVISOR: Dr. Michael S. Patterson

NUMBER OF PAGES: $\mathrm{xi}, 126$ 


\section{ABSTRACT}

A knowledge of the fundamental optical properties of breast tissues is necessary in order to optimize transillumination imaging techniques for the diagnosis of breast disease.

The optical absorption and scattering coefficients have been measured in normal and diseased breast tissues, over the range of wavelengths from 500 to $1100 \mathrm{~nm}$. The tissues were obtained from surgical specimens, and consisted of normal glandular and adipose tissues, fibrocystic disease, fibroadenoma, and ductal carcinoma. Total attenuation coefficients were measured for thin slices of tissue obtained on a microtome. The diffuse reflectance and transmittance were measured for $1.0 \mathrm{~mm}$ thick samples of these tissues, using standard integrating sphere techniques. Monte Carlo simulations were performed to derive the scattering and absorption coefficients, as well as the mean cosine of the scattering angle. The results indicate that scatter exceeds absorption by at least two orders of magnitude. The absorption coefficients are strongly affected by the presence of blood, particularly at wavelengths below 600 $\mathrm{nm}$. The scattering coefficients lie in the range $30 \mathrm{~mm}^{-1}$ to $90 \mathrm{~mm}^{-1}$ at $500 \mathrm{~nm}$, and fall smoothly with increasing 
wavelength to between $10 \mathrm{~mm}^{-1}$ and $50 \mathrm{~mm}^{-1}$ at $1100 \mathrm{~nm}$. The scatter coefficient for adipose tissue differs, in that it is invariant with wavelength over this spectral range. The scattered light, for all tissues examined, is highly forward peaked, with the mean cosine of the scattering angle in the range 0.945 to 0.985 . This value remains constant with wavelength to within $+/-0.01$ for any given tissue.

The absorption coefficients and scattering properties of each tissue type fall within distinct ranges at each wavelength. Fibrocystic disease and adipose tissue appear to be the most clearly distinguishable groups. The optical properties of carcinoma do not differ significantly from those of normal glandular tissues, although both groups differ from other tissue types. The implications of these results for imaging are yet to be determined. 


\section{ACKNOWL EDGEMENTS}

I extend my thanks to my supervisor,

Dr. Michael S. Patterson, and to Dr. Douglas R. Wyman, for suggesting this project, and for the many discussions which have led to its completion.

I am grateful to Dr. B.C.Wilson (Head, Department of Medical Physics and Head of Research, Hamilton Regional Cancer Centre) and to Dr. W.M.Hryniuk (Director, Hamilton Regional Cancer Centre) for providing the opportunity to pursue these studies, while continuing in full time employment with the Cancer Centre. The encouragement (and patience) of the staff of the Radiotherapy and Physics Departments is sincerely appreciated.

I acknowledge the cooperation of Dr. G.L.Frank and the staff of the Department of Anatomical Pathology (Henderson General Hospital, Hamilton) in providing the tissue specimens used in these investigations.

The services provided by the staff of the machine shop and electronics shop, in equipment design and construction, are always valued and appreciated.

Most significant, however, has been the support provided by my wife, Regina, and my children, Jessica and Matthew. I promise that I will not be late for supper as frequently as I have been during the course of this project. 


\section{TABLE OE CONTENTS}

1.1 Breast Cancer.................. 1

1.1.1 Incidence and prognosis...... 1

1.1 .2 screening............... 2

1.2 Anatomy of the breast........... 3

1.2.1 Normal breast structure...... 3

1.2.2 Classification of breast diseases................. 5

1.3 Breast imaging............... 6

1.3.1 General considerations...... 6

1.3.2 Mammography............. 7

1.3.3 Thermography............. 9

1.3 .4 Ultrasonography........... 9

1.3.5 Transillumination.......... 10

1.3.6 Other imaging techniques..... 12

1.4 Optical properties of breast tissues 12

1.4.1 Literature review.......... 12

1.4 .2 Scope of this project....... 13

CHAPTER 2 THEORY OF LIGHT PROPAGATION IN TISSUES

2.1 Introduction................ 16

2.1 .1 Light scattering......... 17

2.1.2 Absorption of light........ 18

2.2 The transport equation........... 19 
Table of contents (continued)

$\underline{\text { Page }}$

2.3 Monte Carlo modeling of light propagation................. 22

2.3 .1 Background.............. 22

2.3.2 Interaction coefficients..... 23

2.3.3 Application to this project... 26

CHAPTER 3 MATERIALS AND METHODS

3.1 Apparatus................. 41

3.2 Calibration of the lamp and monochromator................ 51

3.3 Calibration of the integrating sphere..................... 54

3.4 Reflectance and transmittance measurements................ 59

3.5 Total attenuation measurements..... 61

3.6 Optical properties of Intralipid... 64

3.7 Verification of the mathematical model.................... 65

CHAPTER 4 RESULTS AND DISCUSSION

4.1 Intralipid................. 67

4.2 Polystyrene microspheres........ 73

4.3 Breast tissues............... 80

4.3.1 Effects of tissue preparation. 80

4.3.2 Reflectance and transmittance measurements............. 82

4.3.3 Total attenuation measurements 93

4.3.4 Derived optical properties.... 97 
Table of contents (continued)

4.3.5 Analysis using similarity relationships.............. 102

4.3.6 Phantom materials for breast tissues.................. 109

4.4 Future work.................. 110

4.4.1 Computer simulation of transillumination.......... 110

4.4.2 Development of equipment..... 111

4.4.3 Advanced techniques......... 112

$\begin{array}{lll}\text { CHAPTER } 5 & \text { CONCLUSIONS } & 113\end{array}$

APPENDIX A PROGRAM TO CONTROL THE EXPERIMENT 115

APPENDIX B DERIVATION OF REFLECTANCE AND

TRANSMITTANCE FORMULAE 118

$\begin{array}{ll}\text { BIBLIOGRAPHY } & 123\end{array}$ 


\section{LIST OE FIGURES}

Page

1.1 Lateral view of female breast............... 4

2.1 Plots of the Henyey-Greenstein function........ 25

2.2 Geometry for Monte Carlo simulations........... 27

2.3 Flow chart of the Monte Carlo program......... 31

2.4 Results of the Monte Carlo calculations..... 34-39

3.1 Spectral emission curve for a Xenon arc lamp.... 42

3.2 Optical system used for experiments.......... 43

3.3 Sensitivity of silicon photodiode detectors..... 45

3.4 Apparatus for measuring total attenuation...... 47

3.5 Reflectivity of barium sulfate............. 49

3.6 Integrating sphere geometry for measurement of reflectance and transmittance.............. 50

3.7 Measured spectrum of the 1 ight beam.......... 53

3.8 Calibration of the monochromator............ 55

3.9 Calibration of the integrating sphere......... 58

4.1 Reflectance and transmittance of Intralipid..... 68

4.2 Attenuation measurements for various concentrations of Intralipid............... 69

4.3 Determination of attenuation coefficients for Intralipid............................ 71

4.4 Attenuation coefficients for Intralipid plotted as a function of wavelength................. 72

4.5 Derived optical properties of Intralipid....... 74

4.6 Reflectance and transmittance of polystyrene

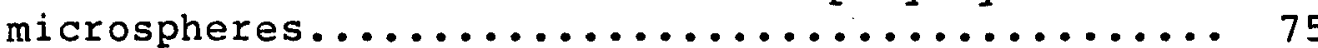


List of figures (continued)

$\underline{\text { Page }}$

4.7 Derived optical properties of polystyrene spheres 77

4.8 Comparison of the scatter coefficients for spheres with values calculated using Mie theory.. 78

4.9 Comparison of the mean cosine of the scattering angle with values calculated using Mie theory.... 79

4.10 Comparison of measured and derived absorption coefficients for suspensions of ink........... 81

4.11 Reflectance and transmittance for each of the five tissue types................... 83-85

4.12 The effect of blood content on the measurements.. 87

4.13 Transmittance plotted as a function of reflectance, for all samples, at $540 \mathrm{~nm} . \ldots \ldots . . .88$

4.14 Transmittance plotted as a function of reflectance at $700 \mathrm{~nm}$ and $900 \mathrm{~nm}$........... 90-92

4.15 Attenuation data for each of the five tissue types, at $700 \mathrm{~nm}$ and $900 \mathrm{~nm} \ldots \ldots \ldots \ldots \ldots \ldots$........94-96

4.16 Derived optical properties for one specimen from each of the five tissue types.......... 98-100

4.17 Sensitivity of the derived optical properties to the value of the total attenuation coefficient... 104

4.18 The transport coefficient plotted as a function of the absorption coefficient, for each sample, at $540 \mathrm{~nm}, 700 \mathrm{~nm}$ and $900 \mathrm{~nm} . \ldots \ldots \ldots \ldots . . .105-107$ 
"We are living in an era of transition in the understanding of breast cancer; yet more changes are yet to come. Implied in this situation is a challenge to push forward the frontiers of diagnostic capability until we can reliably and reproducibly detect noninvasive cancer of the breast and define effective and minimally hazardous ways of treating it."

Robert L. Egan (1972) 
CHAPTER 1

INTRODUCTION

\subsection{Breast cancer}

1.1.1 Incidence and prognosis

Breast cancer is the most common malignancy in women. In Ontario, breast cancer represents 27 percent of all new malignancies diagnosed in females, and accounts for 21 percent of all cancer deaths in females (Clarke et al, 1987). These statistics are in agreement with data available from the United States (Keys et al, 1983), where it is estimated that breast cancer will strike 1 of every 11 females at sometime in life.

Most breast cancers are detected by the patient herself, as a lump in the breast. By this time, approximately 55 percent of these cancers have spread to the axillary lymph nodes. Henderson and Canellos (1980) report a 5 year survival rate of 78 percent for patients who present with tumours which are localized to the breast. If the disease has spread to the axillary lymph nodes, the corresponding survival rate is only 47 percent. Early detection has, therefore, been stressed as a means of improving prognosis. 


\section{$\underline{1.1 .2 \text { Screening }}$}

Screening for breast cancer consists of the regular examination of asymptomatic women. Although palpation is the most direct technique for screening, it lacks the sensitivity required to detect very small lesions. Most screening trials have focused on the use of $x-r a y$ mammography, since the diagnostic value of this technique is undisputed.

Byrd (1980), reporting the results of 28 Breast Cancer Detection Demonstration Projects in the United States, found that 81 percent of all the cancers detected were localized in the breast. This represents a significant improvement in the detection of early stage disease. Other screening programs in Sweden (Tabar et al, 1985) and in New York (Shapiro et al, 1982) have demonstrated a 30 percent reduction in mortality from breast cancer as a result of screening.

Although these results are encouraging, use of mammography must be restricted due to the risk of radiation induced malignancies. Increased public concern with this risk may also cause a reduction in recruitment for this procedure. The development of an effective noninvasive method of detection is desirable, so that screening can be made more widely available. 
1.2 Anatomy of the breast

1.2 .1 Normal breast structure

The imaging of the breast and the diagnosis of breast disease is dependent on an understanding of normal breast anatomy. Differences in the structure of normal breasts may occur from one individual to another, and also for the same individual at different times, depending upon hormonal influences.

The glandular tissues of the breast are enclosed by an outer layer of skin and a subcutaneous layer of fat. Extending from the superficial layers to the chest wall are strands of connective tissue which serve to suspend and compartmentalize the glandular elements. Also contained within the connective tissue are blood vessels and variable amounts of fat. The arrangement and size of the veins vary considerably in different individuals, but are symmetrical in the same individual. The glandular tissues consist of 15 to 20 lobes which subdivide into milk forming lobules, and drain via the ductal system through the nipple. Figure 1.1 is an illustration taken from NCRP Report 85 (1986).

It is the relative amount of fat and glandular tissue that primarily characterizes the normal breast. During the reproductive years, there is a predominance of glandular tissue. Glandular proliferation may be modified by hormonal variations caused by menstruation, pregnancy, and lactation. In general, the glandular tissue recedes 


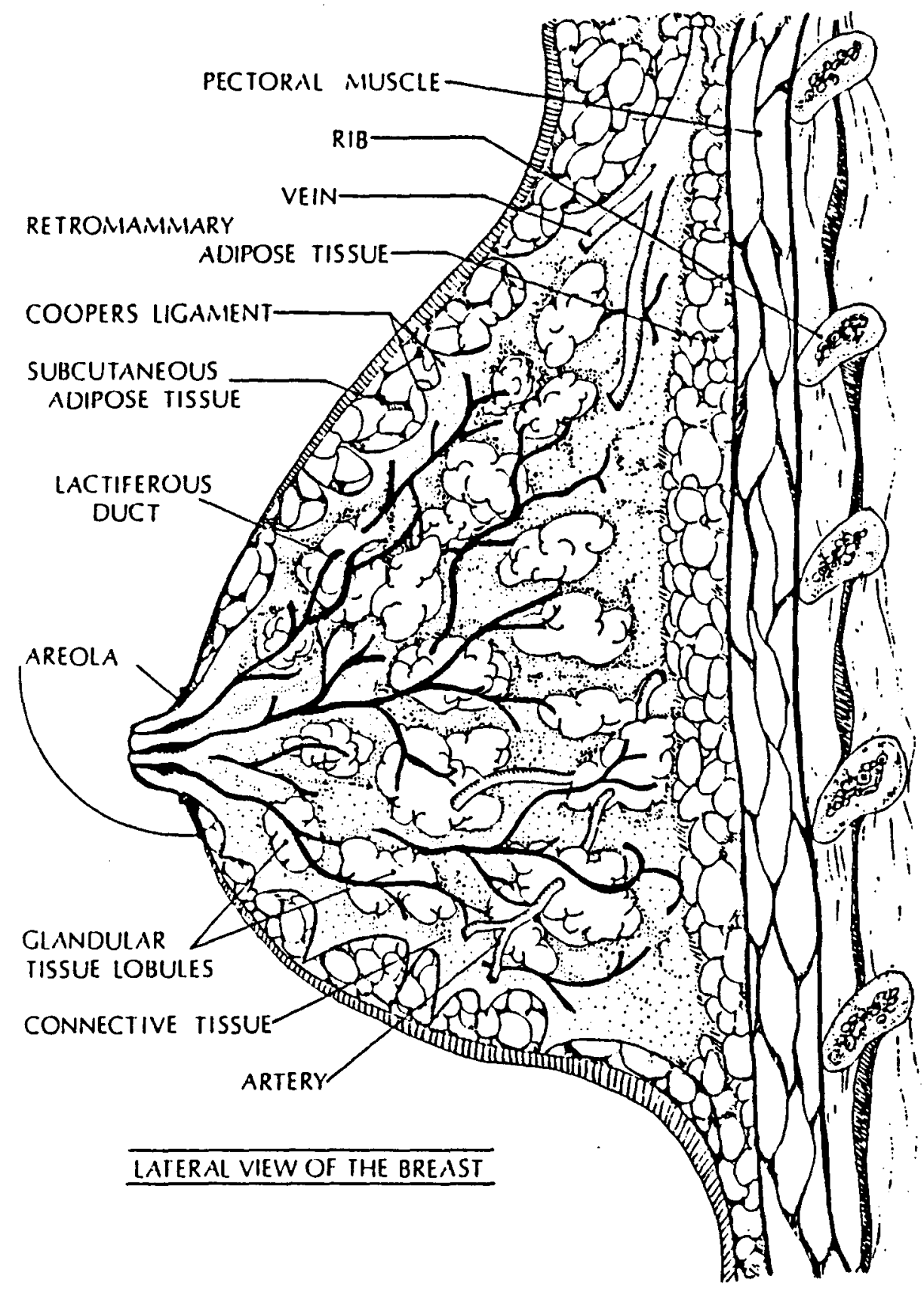

Figure 1.1 Lateral view of female breast. from NCRP Report 85 (1986) 
with increasing age, and is replaced by adipose tissue. In the postmenopausal period the breast may be composed almost entirely of fat.

\subsubsection{Classification of breast diseases}

The most common cause of the lump in the breast is benign breast disease. Byrd (1980) reports that 85 percent of all biopsies, taken as a result of positive findings in mammography or physical examination, are diagnosed as benign conditions.

Fibrocystic disease is the most common benign condition for women under the age of 45 . It is characterized by the development of cysts accompanied by an overgrowth of fibrous tissue. The cysts may vary in size from the microscopic to a centimeter or larger. Fatty deposits are frequently dispersed throughout the area of involvement.

Fibroadenoma is a benign epithelial tumour, and is the most common neoplasm of the breast. These tumours consist of connective and glandular tissues, which may be partially replaced by calcifications. The tumour borders are usually well defined. As with fibrocystic disease, the incidence of fibroadenoma is highest during the reproductive years. 
Most carcinomas of the breast arise in the ductal epithelium. The neoplastic cells are usually identified as clusters within a matrix of fibrous tissue. Increased vascularity is often observed in cancerous breasts and ductal patterns may be asymmetric. Microcalcifications are frequently associated with malignant lesions. In contrast with fibroadenoma, the edges of carcinomas are of ten irregular and may become fixed to adjacent structures.

1.3 Breast imaging

1.3.1 General considerations

The primary goals of breast imaging are to

demonstrate the presence or absence of an abnormality, and to indicate its size and location. The method should be as sensitive as possible, so that the diagnosis can be made at an early stage of the disease. The method should also be able to differentiate benign and malignant lesions, and ideally, will make possible a specific histologic diagnosis.

Screening techniques should be noninvasive and, as far as possible, should present no risk to the patient. This requirement may be relaxed for the diagnostic examination of symptomatic women, because of the clinical need for the information obtained. 
A brief review of breast imaging techniques is provided in the following sections. Further details and references can be obtained from Jones (1982), Isard (1984), and NCRP (1986)

\section{$\underline{1.3 .2 \text { Mammography }}$}

$\mathrm{X}$-ray mammography is currently the only technique which is recognized as having sufficient sensitivity for use in a screening program (Day and Miller, 1988). Fagerberg et al (1985) estimated that single view mammography can lead to detection of breast cancers an average of 3.5 years prior to clinical presentation. The basis of this imaging method is the differential attenuation of $x$-rays in the tissues of the breast. Since fibrous and glandular tissues have approximately the same mass density and atomic number, they cannot be adequately separated on mammograms (Egan, 1972) . At the $x$-ray energies used in mammography, the attenuation coefficients for carcinoma are only slightly higher than those for fibrous tissues (Johns and Yaffe, 1987). These structures are more easily identified when separated by layers of adipose tissue which is less attenuating. Therefore, breasts with minimal amounts of fat, as in young adults, present the greatest diagnostic problems. 
Localized breast lesions are usually diagnosed by the radiographic appearance of the mass. Calcifications, increased vascularity, or asymmetry in the pattern of veins may also be indications of breast disease. Egan (1972) and Wolfe (1972) provide detailed information on the radiographic diagnosis of breast disease using screen-film mammography and xeroradiography respectively. These two techniques produce images of similar quality and are both in current clinical use.

Xeroradiography utilizes a charged photoconductive plate as a detector, thus forming an electrostatic image of the transmitted $x-r a y$ beam. The plate is subsequently dusted with a toner and the resultant powder image is transferred to paper. The main advantages of this technique are edge enhancement and a wider latitude than film.

Using current techniques and equipment, the average glandular dose for two view xeromammography or screen-film mammography is between 0.1 and $0.8 \mathrm{cGy}$. For these doses, the benefit/risk ratio is favourable for the annual screening of women of age 40 and up (NCRP, 1986). For those techniques that deliver 0.1 to $0.2 \mathrm{cGy}$ the benefit/risk ratio may be favourable down to age 30 . However, for younger women, and for those who require more frequent examination, the risk remains unacceptably high. 
Other radiographic techniques, such as ionography and digital mammography, are under investigation in an attempt to further reduce radiation exposures and improve images (NCRP, 1986).

\subsubsection{Thermography}

Thermography is a method of measuring the temperature distribution on or near the surface of the breast. This is usually done using infrared detectors having peak sensitivities at wavelengths between 5 and 20 microns. It is based on the observation that tumours may cause an increase in surface temperature as a result of changes in the superficial vasculature or differences in tumour metabolism.

The advantage of thermography is that it is completely safe and can be used freely in conjunction with other techniques. However, thermography lacks the sensitivity required for a screening procedure, since thermal changes are most often associated with advanced cancer.

\section{$\underline{1.3 .4 \text { Ultrasonography }}$}

In ultrasonography, short pulses of high frequency sound are directed into the breast from piezoelectric transducers. This energy is partially reflected at tissue interfaces due to changes in the acoustic impedance. 
The reflected pulses are detected and analyzed, using a time of flight method, to determine the depths of the interfaces. Cross-sectional images are constructed by scanning the transducers, or through use of multiple detectors.

This technique has no known biological risks, and has the advantage of being able to distinguish cystic from solid masses. In contrast with mammography, ultrasound is more effective in demonstrating disease in dense, glandular breasts than in those consisting primarily of fat. Although ultrasound may have value as an adjuvant procedure, it is not used as a primary screening modality due to the difficulty of resolving early stage disease.

\subsubsection{Transillumination}

Transillumination (diaphanography) is the process by which images are formed by light transmitted through the breast.

The technique was originally used for direct viewing of the shadows cast when the breast was transilluminated with an intense light beam (Cutler, 1929). More recently, the use of near-infrared wavelengths has been shown to improve tumour visibility. In this case, images are recorded on infrared-sensitive photographic film (Ohlsson et al, 1980), or viewed in real time using television cameras sensitive to near-infrared radiation. 
Image formation depends upon the differential absorption and scattering of light. The size, depth and optical properties of a lesion, as well as of surrounding tissues, influence tumour visibility. The shadow of a mass is clearly seen if it is large or lies near the surface of the volume being imaged; small, deep lesions are more difficult to detect due to multiple scattering. Since hemoglobin has strong absorption bands at wavelengths below 600 nanometers, it has been speculated that the detection of tumours at visible wavelengths may be largely due to increased vascularity. It has not yet been determined whether blood content is the primary cause of contrast at other wavelengths. Recently developed diaphanography systems have the capability of presenting transmission data in two or more spectral bands (Profio et a1, 1988) .

Transillumination is a risk-free method of evaluating breast disease. It is useful in distinguishing cystic from solid lesions and is particularly valuable in the diagnosis of hematoma. It has been able to detect some cancers which were not demonstrated by mammography (Wallberg, 1985) and, therefore, may be a useful complementary procedure.

However, transillumination is still considered an experimental technique. A better understanding of the basic optical properties of breast tissues is required in order 
to select the optimal equipment and methods of spectral analysis.

\subsubsection{Other imaging techniques}

virtually all diagnostic modalities have been investigated for use in breast imaging. Radionuclide studies are invasive and do not provide sufficient resolution. Computed tomography is not advocated for screening because the radiation dose is relatively high, and the procedure requires the use of an intravenous contrast agent. Heavy ion mammography and magnetic resonance imaging have shown promise; however, the high cost will likely preclude their use for screening.

1.4 Optical properties of breast tissues 1.4.1 Literature review Studies of transillumination have usually focused on the clinical evaluation of specific source-detector systems. Until very recently, there have been no quantitative studies of the propagation of red and infrared light through breast tissues.

In 1982, Carlsen suggested that there may be a difference between the spectral attenuation of cancerous and normal breast tissues. However, Watmough (1982) was unable to demonstrate a correlation between transmittance and breast pathology. Ertefai and Profio (1985) also 
concluded that the transmittance of cancerous and glandular breast tissues showed little difference over the range 600 to 1060 nanometers. They did, however, observe greater transmittance for adipose tissue in the red region of the spectrum. All of these authors demonstrated increased attenuation in tissues with high blood content, and speculated that the detection of tumours may be due to increased vascularity. The results of these studies are inconclusive due to the small numbers of breast specimens examined. Only Ertefai and Profio (1985) attempted to model the light tissue interaction process; however, the absorption and scattering coefficients, which they obtained using Kubelka-Munk theory (Kortum, 1969), are derived under the assumption of isotropic scattering in an infinite medium. These effective coefficients are not directly comparable with the fundamental interaction coefficients defined in the following chapter.

\subsubsection{Scope of this project}

In this project, the optical characteristics of normal and diseased breast tissues are investigated for wavelengths in the range 500 to 1100 nanometers. The transmittance, reflectance, and total attenuation are measured for excised breast specimens. A Monte Carlo method is used to derive the absorption and scattering coefficients, as well as the mean scattering angle. 
This work is part of a larger research program, which is being conducted at the Hamilton Regional Cancer Centre of the Ontario Cancer Treatment and Research Foundation. Investigations into transillumination are proceeding on four fronts as follows:

1) Determination of fundamental light-tissue interaction parameters.

2) Development of breast phantoms to permit physical simulations of real examinations.

3) Development of mathematical models to allow computer simulations in differing geometries.

4) Investigation of time gating to reject scatter. Part (1) is the subject of this report. Part (2), the development of suitable phantoms, has been reported by Hansen (1988). Phantom results were obtained for a single wavelength using a helium-neon laser (633 nm). The development of appropriate materials will continue, based on the measured optical properties of breast tissues.

Part (3) of the research program will focus on mathematical modelling of the imaging process. A Monte Carlo code has been written to simulate light propagation in thick media (Wyman et al, 1989). Using the measured Iight-tissue interaction parameters, the Monte Carlo results will be compared with the phantom data at $633 \mathrm{~nm}$. Having tested the accuracy of the code, simulations will be 
performed at other wavelengths, and for other geometries.

Part (4) will examine the use of time gated

detectors as a means of rejecting scatter. This technique is based on the fact that scattered photons take more time to reach the detector than photons which travel along a direct path.

The ultimate goal of these investigations is to determine the optimal technique for transillumination, using optimized imaging spectra and scatter rejection methods. 


\section{CHAPTER 2}

\section{THEORY OF LIGHT PROPAGATION IN TISSUES}

\subsection{Introduction}

In order to study the image formation process in transillumination, we require an understanding of light propagation and absorption in tissues.

Electromagnetic radiation can be described as waves or particles depending upon the wavelength of the radiation and the size of the structures with which it interacts. In the case of large homogeneous objects, geometrical optics is sufficient to determine the direction in which light travels. The wave nature is most suitable for describing the propagation through nonabsorbing media in which the size and spacing of the structures are comparable with the wavelength. This propagation is governed by Maxwell's equations, from which the principles of reflection, refraction, and diffraction can be derived. on a macroscopic scale the attenuation of light in a semi-infinite medium irradiated with a plane parallel beam of light can be described by

$$
\phi(z)=\phi(0) \exp \left(-\sum_{e f f} \cdot z\right) 2.1
$$


where $\phi(0)$ is the incident photon fluence rate, $\boldsymbol{\phi}(\boldsymbol{z})$ is the photon fluence rate at depth, $\boldsymbol{z}$ and $\sum$ eff is an effective attenuation coefficient. The photon fluence rate represents the total number of photons per unit time which pass through a sphere of unit cross-sectional area and includes all scattered photons. $\sum_{\text {eff }}$ is only equal to the total attenuation coefficient, $\Sigma_{+}$, for a purely absorbing material since $\Sigma_{+}$corrects for all scattering and absorption losses. The use of an effective attenuation coefficient is inadequate in describing light transport in a narrow beam, in inhomogeneous systems or near any boundary of a scattering medium.

\section{$\underline{2.1 .1 \text { Light scattering }}$}

In this study, the wavelengths of light considered lie in the range 500 to 1100 nanometers. These wavelengths are smaller than the cells and some intracellular components which make up biological tissues.

Light scattering results when the electromagnetic field induces oscillations in the molecular electrons, which subsequently act as sources of light. The scattered photons have the same energy as the incident radiation. Interference will occur between light waves which are generated in different parts of the object. For a light beam incident on a surface between two homogeneous media, 
this interference results in reflection and refraction. Mie theory has been used to describe scattering for small spherical particles; however, the complexity of cellular structure makes it difficult to predict the result of scattering processes in a biological material.

\subsubsection{Absorption of light}

Absorption is the transfer of energy from the photons to the molecules of the medium. The absorption of individual photons depends upon the characteristic vibrational, rotational and electronic energy states of the molecules with which they interact. If the wavelength of the electromagnetic radiation is close to the natural period of oscillation of one of these states, then the probability of absorption is high. The absorbed energy is largely converted to heat, although it may also contribute to chemical transformations. The absorption spectrum of each molecule is characteristic of all the functional groups present in the molecule and as such, can be used in identifying the molecule. The local molecular environment may cause small shifts in the energy levels, which serve to broaden the absorption peaks into bands. Since biological cells are complex aggregates of thousands of molecular types, it is not possible to determine the exact composition from the combined absorption spectra. 


\subsection{The transport equation}

The solution of Maxwell's equations for light propagation in a highly inhomogeneous medium is not feasible (Wilson and Patterson, 1986). An alternate method is the application of the concept of particle transport, as developed to describe the propagation of neutrons in a reactor (Duderstadt and Hamilton, 1976). In this approach, the light photons are treated as neutral particles; polarization may be included although diffraction effects are ignored. The Boltzman transport equation governs neutral particle transport and is a statement of the balance in the number of particles in an arbitrary volume in phase space. A description of its application in photon transport is given by wilson and Patterson (1986), and is reviewed below to clarify some of the concepts which will be used later.

We are primarily interested in determining the photon angular density, $n(\bar{r}, \bar{\Omega})$, which is defined such that $n(\bar{r}, \bar{\Omega}) \overline{d r} \sqrt{\Omega}$ is the expected number of photons in $\overline{d r}$ about $\bar{r}$ moving in a solid angle $\overline{d \Omega}$ about the direction $\bar{\Omega}$. It is assumed that a steady state condition has been established so that there is no time dependence for $n$. It is further assumed that the photons are monoenergetic, and that the scattering process is elastic. 
The photon angular flux is defined by :

$$
\phi(\bar{r}, \bar{\Omega})=v \cdot n(\bar{r}, \bar{\Omega}) \quad 2.2
$$

where $\checkmark$ is the speed of light in the medium.

To describe photon transport, it is necessary to define the photon interaction coefficients. $\sum_{a}(\vec{F})$ and $\sum_{S}(\bar{r})$ represent the probabilities per infinitesimal path length that a photon will be absorbed or scattered, respectively. The total attenuation coefficient is given by : $\quad \sum_{+}(\bar{r})=\Sigma_{a}(\bar{r})+\sum_{s}(\bar{r}) \quad 2.3$ The differential scattering coefficient, $\sum_{s}\left(\bar{r}, \bar{\Omega}^{\prime} \rightarrow \bar{\Omega}\right)$, represents the probability per infinitesimal path length per unit solid angle that a photon moving in direction $\bar{\Omega}^{\prime}$ at $\bar{r}$ will be scattered to the new direction $\bar{\Omega}$. The total scattering coefficient is given by the integral of the differential coefficient over all final $\begin{aligned} & \text { directions : } \sum_{s}(\bar{r})=\int_{4 \pi} \sum_{s}\left(\bar{r}, \bar{\Omega}^{\prime} \rightarrow \bar{\Omega}\right) d \bar{\Omega} \quad 2.4 \\ & \Sigma_{a}(\bar{r}) \text { and } \sum_{S}(\bar{r}) \text { are assumed to be independent }\end{aligned}$ of the original directions of the photon. This is reasonable for a random material but may not be true for some biological tissues (Wilksch et al, 1984).

The transport equation can be stated as :

$$
\begin{aligned}
\bar{\Omega} & \cdot \overline{\nabla \phi}(\bar{r}, \bar{\Omega})+\Sigma_{+}(\bar{r}) \cdot \phi(\bar{r}, \bar{\Omega}) \\
& =\int_{4 \pi} d \bar{\Omega}^{\prime} \Sigma_{s}\left(\bar{r}, \bar{\Omega}^{\prime} \rightarrow \bar{\Omega}\right) \cdot \phi\left(\bar{r}, \bar{\Omega}^{\prime}\right)+C(\bar{r}, \bar{\Omega}) 2.5
\end{aligned}
$$


The various terms in the transport equation are explained as follows :

$\bar{\Omega} \cdot \bar{\nabla} \phi(\bar{r}, \bar{\Omega})$ represents the net flux of photons out of the volume element in the direction $\bar{\Omega}$. (i.e. the flux of photons which leaves the volume in the direction $\bar{\Omega}$ minus the flux of photons entering the volume with direction $\bar{\Omega}$,

$\Sigma_{+}(\bar{r}) \cdot \phi(\bar{r}, \bar{\Omega})$ represents the loss of photons within the volume element, due to absorption or scattering away from the original propagation direction $\bar{\Omega}$.

$$
\int_{4 \pi} d \bar{\Omega}^{\prime} \sum_{s}\left(\bar{r}, \bar{\Omega}^{\prime} \rightarrow \bar{\Omega}\right) \cdot \phi\left(\bar{r}, \bar{\Omega}^{\prime}\right) \text { represents all the photons }
$$

which are originally travelling in directions other than $\bar{\Omega}$ when they enter the volume element, but are subsequently scattered into this direction.

$C(\bar{r}, \bar{\Omega})$ represents a source of photons within the volume element.

Analytical solutions of this equation are only possible for a few simple geometries. Approximate solutions can be obtained under certain conditions using diffusion theory (Duderstadt and Hamilton, 1976); however, this approach is invalid near boundaries, due to the assumption of a nearly isotropic angular flux. The solution for a finite beam and a three dimensional geometry containing inhomogeneities requires a numerical approach. 
2.3 Monte Carlo modeling of light propagation $\underline{2.3 .1}$ Background

Monte Carlo techniques have been widely used in the study of radiation transport. Essentially, these methods trace the histories of individual particles. The basic assumption is that each interaction can be simulated by random sampling of interaction parameters from appropriate probability distributions. In order to reach general conclusions, a large number of particle histories must be examined. A summary of the application of Monte Carlo in particle transport is given by Carter and Cashwell (1975) •

Only recently have Monte Carlo methods been used to study light propagation in tissues. Wilson and Adam (1983) used this technique to predict photon flux distributions in homogeneous media for various source geometries. Wilksch et al (1984) derived fundamental optical properties of animal tissues by matching measured reflectance and transmittance values with those obtained by Monte Carlo simulation.

In principle, the Monte Carlo method can be used to deal with light propagation in a medium of any given complexity. Computation times, however, present practical limits to the solution of very complex problems. Wyman and Patterson (1988) and Wyman et al (1989) have introduced methods to improve the efficiency of calculations in media 
which are many mean free paths in thickness. Incorporation of such methods is essential in modeling the image formation process in transillumination.

\subsubsection{Interaction coefficients}

In order to solve the transport equation

numerically for the photon angular flux, $\phi(\bar{r}, \bar{\Omega})$, we need to know the absorption and scattering coefficients of the tissues examined, as well as the differential scattering coefficient. Related quantities are the single scattering albedo, $a$, and the scattering phase function $S\left(\bar{r}, \bar{\Omega}^{i} \rightarrow \bar{\Omega}\right)$. The albedo is the ratio of the scattering coefficient, $\Sigma_{S}(\bar{r})$, to the total attenuation coefficient, $\Sigma_{+}(F)$. The scattering phase function is equal to the differential scattering coefficient, normalized such that integration over all angles gives unity.

$$
\begin{array}{rl} 
& S\left(\bar{r}, \bar{\Omega}^{\prime} \rightarrow \bar{\Omega}\right)=\sum_{S}\left(\bar{r}, \bar{\Omega}^{\prime} \rightarrow \bar{\Omega}\right) / \Sigma_{S}(\bar{r}) \\
\therefore S_{4 \pi} S\left(\bar{r}, \bar{\Omega}^{\prime} \rightarrow \bar{\Omega}\right) d \bar{\Omega}=1 & 2.6
\end{array}
$$

We will assume that the scattering centers are randomly oriented, so that $S\left(\bar{F}, \bar{\Omega}^{\prime} \rightarrow \bar{\Omega}\right)$ is a function only of the angle, $\theta$, between the incident direction, $\Omega^{\prime}$, and the exit direction, $\bar{\Omega}$. The simplified scatter phase function, $S(\bar{r}, \theta)$, describes the angular dependence of the scattering : $S(\bar{r}, \theta)=2 \pi S\left(\bar{r}, \bar{\Omega}^{\prime} \rightarrow \bar{\Omega}\right) \quad 2.8$ The factor $2 \pi$ accounts for azimuthal integration, assuming azimuthal symmetry. 
Although the actual scatter phase functions may be quite complex, it is often possible to replace these functions with simpler forms. One which is frequently used to examine the influence of anisotropy in light scattering is the Henyey-Greenstein function (van de Hulst, 1980) given by :

$$
S(\theta)=0.5 *\left(1-g^{2}\right) /\left(1+g^{2}-2 g \cos \theta\right)^{3 / 2} \quad 2.9
$$

where $g$ represents the mean cosine of the scattering angle and is given by :

$$
g=\int_{-1}^{1} S(\theta) \cos \theta d(\cos \theta)
$$

The dependence of $g$ and $S(\theta)$ on $\bar{r}$ is implied. The value of $g$ can be varied to change the shape of this function from one which is purely isotropic $(g=0)$, to one which is sharply forward peaked (see Figure 2.1(a)). The scatter functions for some animal tissues have been measured by Flock et al (1987) and wilksch et al (1984). Some of the data from Flock et al is reproduced in figure $2.1(b)$. Henyey-Greenstein functions, corresponding to the measured $g$ values, are also plotted for comparison. Within experimental uncertainties, these functions provide a good fit to the data. In this project, the scatter phase function will be assumed to be represented by a Henyey-Greenstein function. 
a)<smiles></smiles>

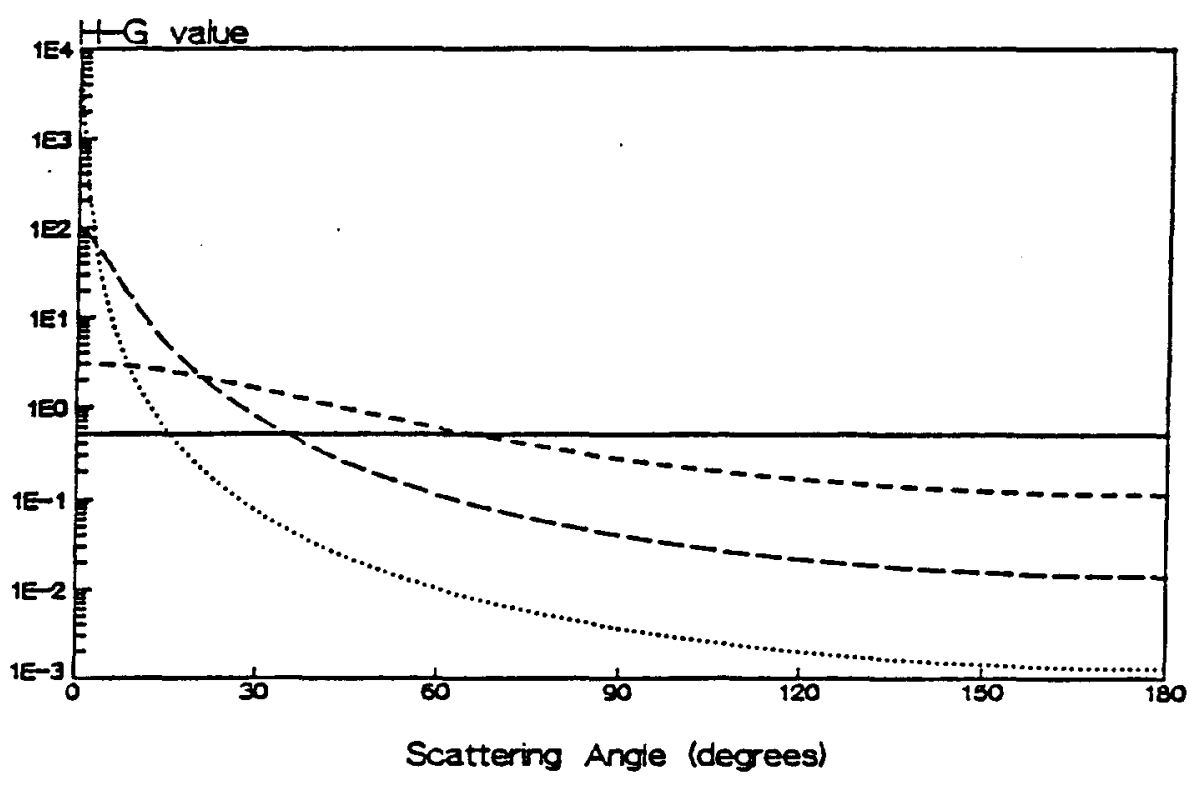

b)

$$
\begin{aligned}
& -H G \text { function } \\
& g=0.771
\end{aligned}
$$

$H G$ function $g=0.941$

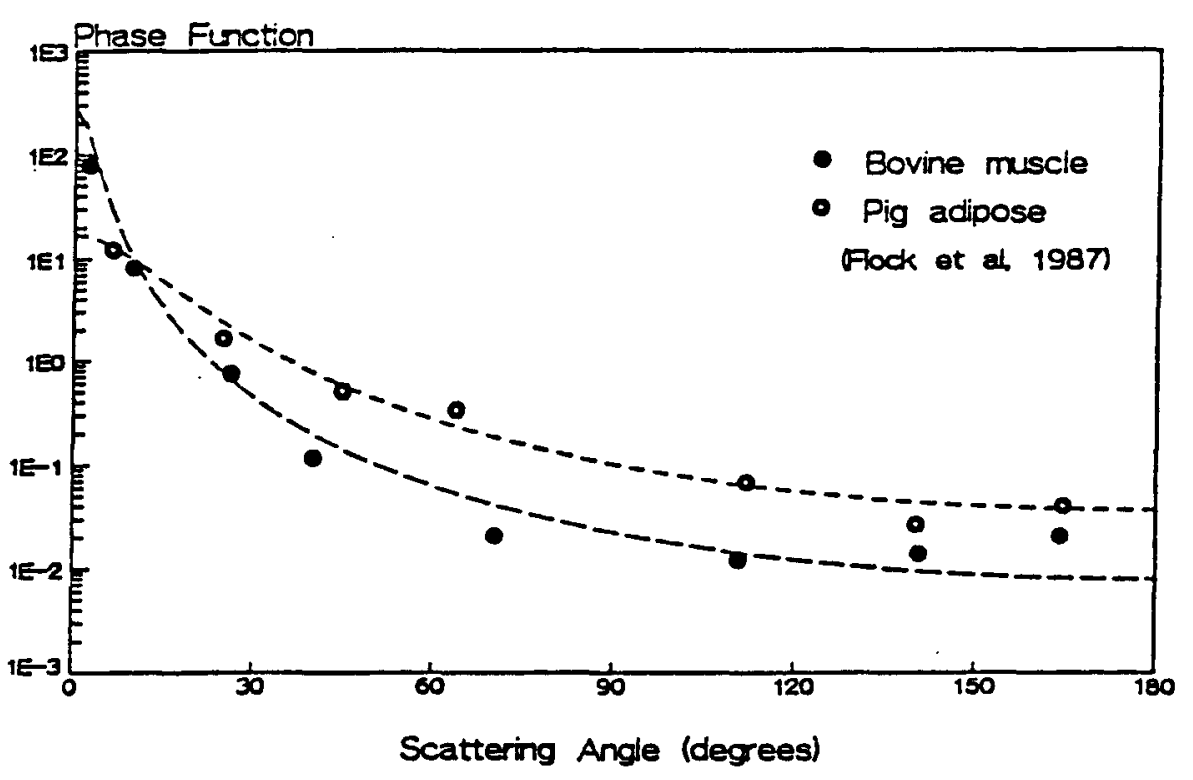

Figure 2.1 a) Plots of the Henyey-Greenstein function with four different values of the mean cosine of the scattering angle, 'g'. b) Measured scatter phase functions for bovine muscle and pig adipose (Flock et al, 1987). The smooth curves are the HenyeyGreenstein functions which correspond to the measured ' $g$ ' values. 


\subsubsection{Application to this project}

In this project, we will apply the Monte Carlo method to an inverse problem, which is to derive the interaction coefficients from measured reflectance and transmittance. More specifically, we will derive the albedo, $a$, and the mean cosine of the scattering angle, $g$. As will be described in chapter 3, measurements have been made on excised breast specimens, $1.0 \mathrm{~mm}$ in thickness, to determine the diffuse reflectance, $R_{M}$, and diffuse transmittance, $T_{M}$. The total attenuation coefficient, $\Sigma_{+}$(tissue), is also directly measured for thin sections taken from these same samples.

The Monte Carlo program, used to simulate the reflectance and transmittance measurements, was written by Dr. D. Wyman at this institution and modified by this author. The irradiation geometry is shown in figure 2.2. A parallel beam of light of diameter $d$, is assumed to be incident normally on the sample, which is held between $1 \mathrm{~mm}$ thick glass slides. The light beam is further assumed to have a uniform intensity distribution. The light emerging through the apertures of diameter $D$, on the entrance and exit faces of the sample holder, is assumed to be detected. The calculated transmittance, $T_{c}$, is that fraction of the incident flux which emerges through the exit face of the sample holder. It includes both unattenuated photons and forward scattered photons. The calculated reflectance, $R_{c}$, 


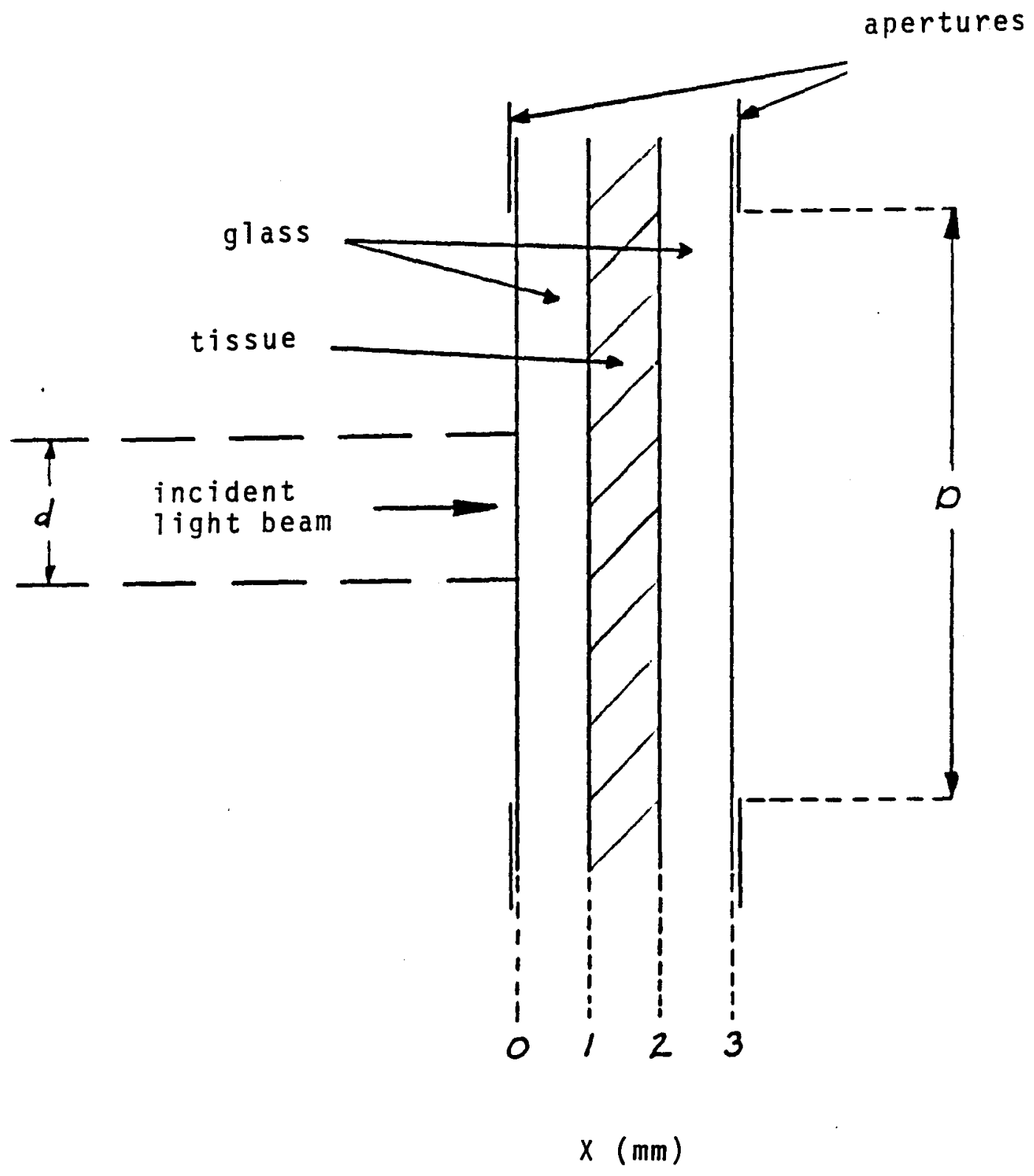

Figure 2.2 Geometry for the Monte Carlo simulations. 
is that fraction of the incident flux which is backscattered through the aperture on the entrance face of the sample holder. The reflectance does not, however, include the incident photons which are specularly reflected on first encounter with the air-glass interface.

The index of refraction for crown glass is taken to be 1.5 (CRC Handbook of Chemistry and Physics, 1983-1984). For our simulations, we assumed that the refractive index of tissue is identical to that of water (1.33). Recent data (Bolin et al, 1989) indicate that the refractive index of tissue may lie closer to the value 1.40. Possible errors resulting from this difference are discussed at the end of this section.

At tissue-glass interfaces, photons are assumed to change direction due to the mismatch in refractive index; however, specular reflections are ignored. This should introduce little error since the reflected intensity is approximately an order of magnitude smaller than that which occurs at glass-air interfaces. The refraction angle is calculated from snell's law:

$$
\sin (r)=\left(n_{1} / n_{2}\right) \cdot \sin (i) \quad 2.11
$$

where $i$ is the angle of incidence, and $r$ is the angle of refraction (both angles being measured with respect to the normal to the surface),

and $n_{1}$ and $n_{2}$ are the indices of refraction for photons moving from medium 1 to medium 2 . 
The fraction of light reflected at glass-air interfaces is calculated using the Fresnel reflection coefficients for light polarized in a plane parallel or perpendicular to the plane of incidence. For parallel polarization, the reflected fraction of the incident intensity is given by :

$$
R_{11}=\tan ^{2}(i-r) / \tan ^{2}(i+r)
$$

For perpendicular polarization, the reflected fraction is :

$$
R_{\perp}=\sin ^{2}(i-r) / \sin ^{2}(i+r)
$$

The actual reflection value, used in the Monte Carlo code, is the average of the two calculated values. This assumes that the scattered light is randomly polarized (Bohren and Huffman, 1983). For incident angles greater than 41.8 degrees, the light is totally reflected, when medium 1 is glass $\left(n_{1}=1.5\right)$ and medium 2 is air $\left(n_{2}=1.0\right)$. For normal incidence the specular reflection loss at a glass-air interface is 4.0 percent.

The attenuation coefficient for glass, $\Sigma_{+}$(glass), was required as an input parameter in the Monte Carlo code. This value was taken to be zero, based on attenuation measurements described in chapter 3. 
A simplified flow chart of the Monte Carlo code is provided in figure 2.3. As already stated, the input parameters include $d, D, \Sigma_{+}$(tissue), $\Sigma_{+}$(glass), $R_{M}$ and $T_{M}$ as previously defined. Also entered are starting values for the albedo, $a$, and the mean cosine of the scattering angle, 9 . Each photon history begins with the random selection of its position within the beam at $x=0$ mm. A 4.0 percent loss for specular reflection is assumed. Pathlengths in glass and tissue, $\ell_{j}$, are randomly selected using a cumulative distribution sampling method (Carter and Cashwell, 1975), according to:

$$
\ell_{j}=-\ln \left(N_{j}\right) / \Sigma_{+}
$$

where $N_{j}$ is a randomly generated number between 0 and 1 , and $\Sigma_{+}$is the attenuation coefficient for tissue or glass as required. When the path of a photon crosses a regional boundary, its position is reset to the point at which it intersects the boundary. A new pathlength is calculated in the next medium, and the direction is reset according to the angle of refraction. Interactions in tissue are selected randomly as scattering or absorption events, according to the albedo. The scattering angle, $\theta$, is randomly sampled from the Henyey-Greenstein function. The azimuthal angle is randomly and uniformly sampled between 0 and $2 \pi$. At glass-air interfaces, the photon either exits the sample holder or is specularly reflected. If the photon leaves the sample holder within the diameter of the 


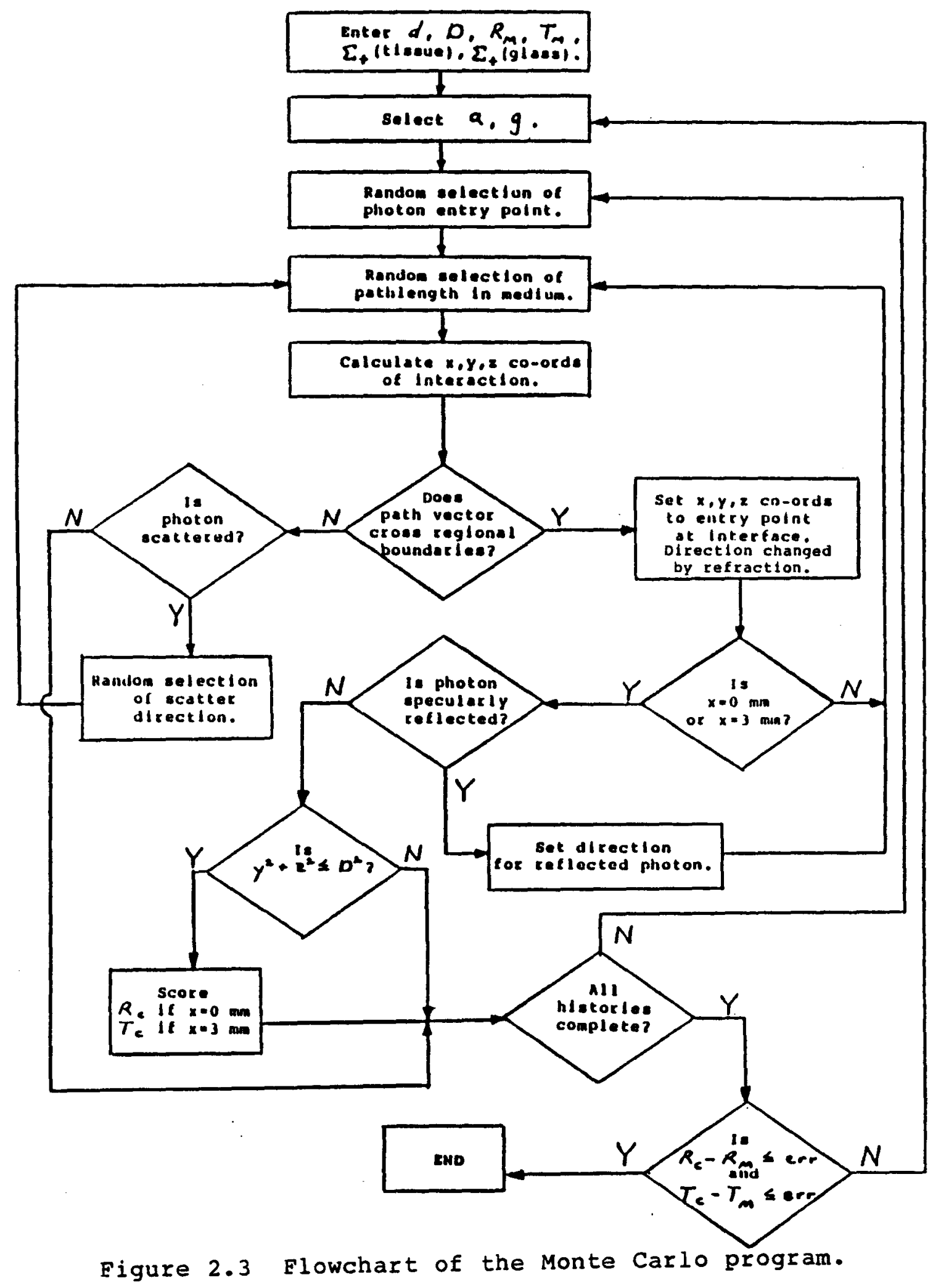


aperture, $D$, then the photon is scored as reflected or transmitted depending on the surface through which it exits. New incident photons are selected up to a predetermined limit. At this point, the calculated reflectance, $R_{c}$, and transmittance, $T_{c}$, are compared with the corresponding measured quantities. New values of $\boldsymbol{a}$ and $g$ are selected and the entire program repeated, until an acceptable level of agreement is achieved. The above description applies to the basic flowchart of figure 2.3. To improve computation efficiency, survival weighting was used for each interaction. That is, an incident photon with a weight of 1.0 is assumed to lose a fraction of this weight at each interaction as a result of absorption. The scattered weight is, therefore, equal to the product of the albedo with the weight of the photon prior to the interaction. For interactions at glass-air interfaces, the average Fresnel reflection coefficient replaces the albedo in this product. The weighted fraction which crosses the glass-air interface within the limits of the detector aperture is scored as reflected or transmitted. The history of the scattered fraction continues until the surviving weight falls below a small value which was chosen such that the errors introduced by this loss are negligible. 
These calculations can be very time consuming if the entire process is repeated at a number of wavelengths for each sample. To simplify the process, the Monte Carlo program was run for a selected set of the parameters $\Sigma_{+}$, $a$, and $g$. The beam diameter, $d$, and aperture size, $D$, were taken to be $3.0 \mathrm{~mm}$ and $12.7 \mathrm{~mm}$ to correspond to the experimental conditions. The results are presented in figure 2.4. For each value of $\alpha$ and $g$, the calculated reflectance, $R_{c}$, (or transmittance, $T_{c}$ ) is plotted as a function of the total attenuation coefficient. Families of curves corresponding to various $g$ values are plotted on a single graph. Presented in figure 2.4 are graphs corresponding to $\alpha$ values of $1.0, .998, .995, .990, .980$, and .960 . For any given sample, the measured reflectance, $R_{M}$, combined with the measured attenuation coefficient, $\Sigma_{+}$, can be used to obtain pairs of compatible values of $a$ and $g$ from these graphs. Similarly, the transmittance, $T_{M}$, combined with $\Sigma_{+}$can be used to obtain a second set of acceptable pairs. The correct values of $a$ and $g$ are the only mutually acceptable values, interpolated from the paired data sets. 
mean cosine of the scattering angle $=$

$+.60-\Delta-.80-0 . .90 \cdots+\cdots .95 \rightarrow .98 \rightarrow-.99+.995$
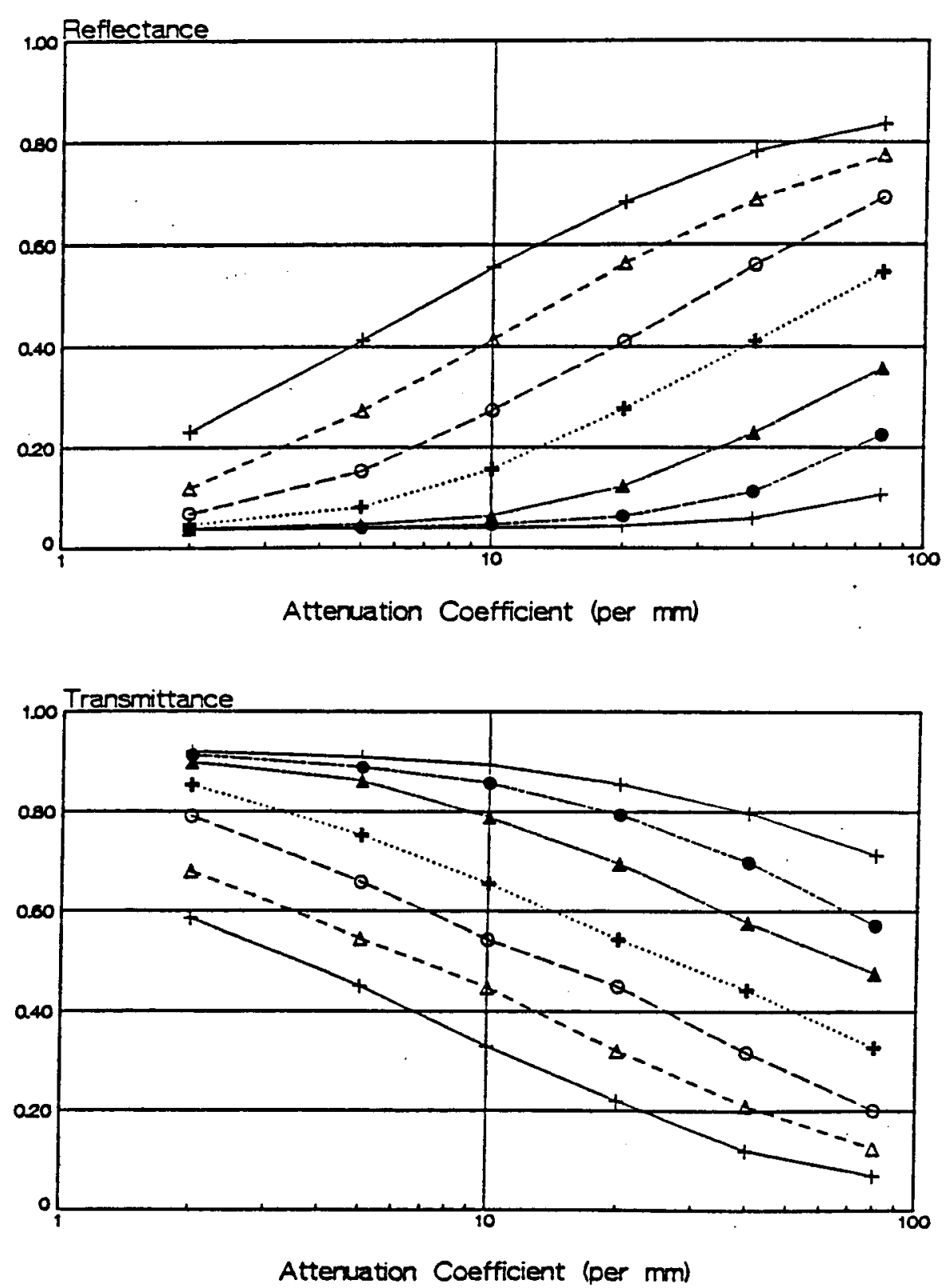

Figure 2.4 The reflectance and transmittance, as determined from the Monte Carlo model, plotted as functions of the total attenuation coefficient for a range of ' $g$ ' values. a) Albedo $=1.000$ 
mean cosine of the scattering angle $=$ $+.60-\Delta-.80-0.90 \cdots+\cdots .95 \rightarrow .98 \rightarrow-.99 \div-.995$

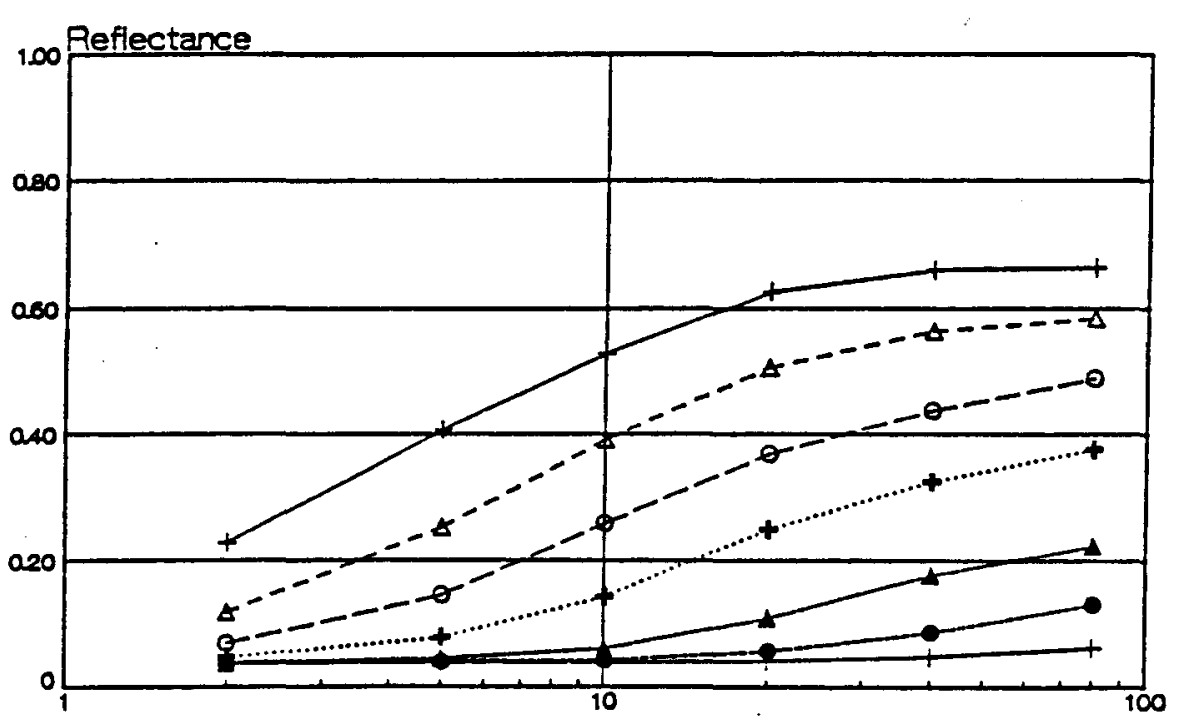

Attenuation Coefficient (per mm)

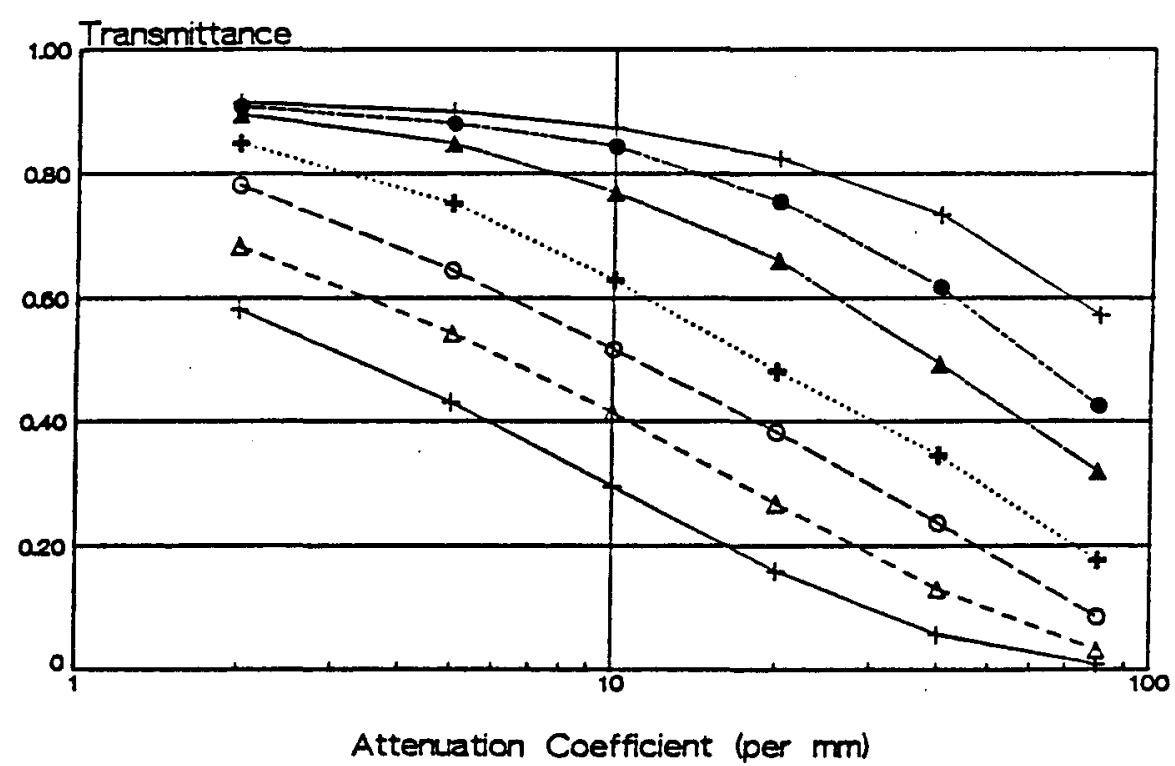

Eigure 2.4 (continued)

b) Albedo $=0.998$ 
mean cosine of the scattering angle $=$ $+.60-\Delta-.80 \rightarrow-.90 \cdots+\cdots .95 \rightarrow .98 \rightarrow-.99+.995$
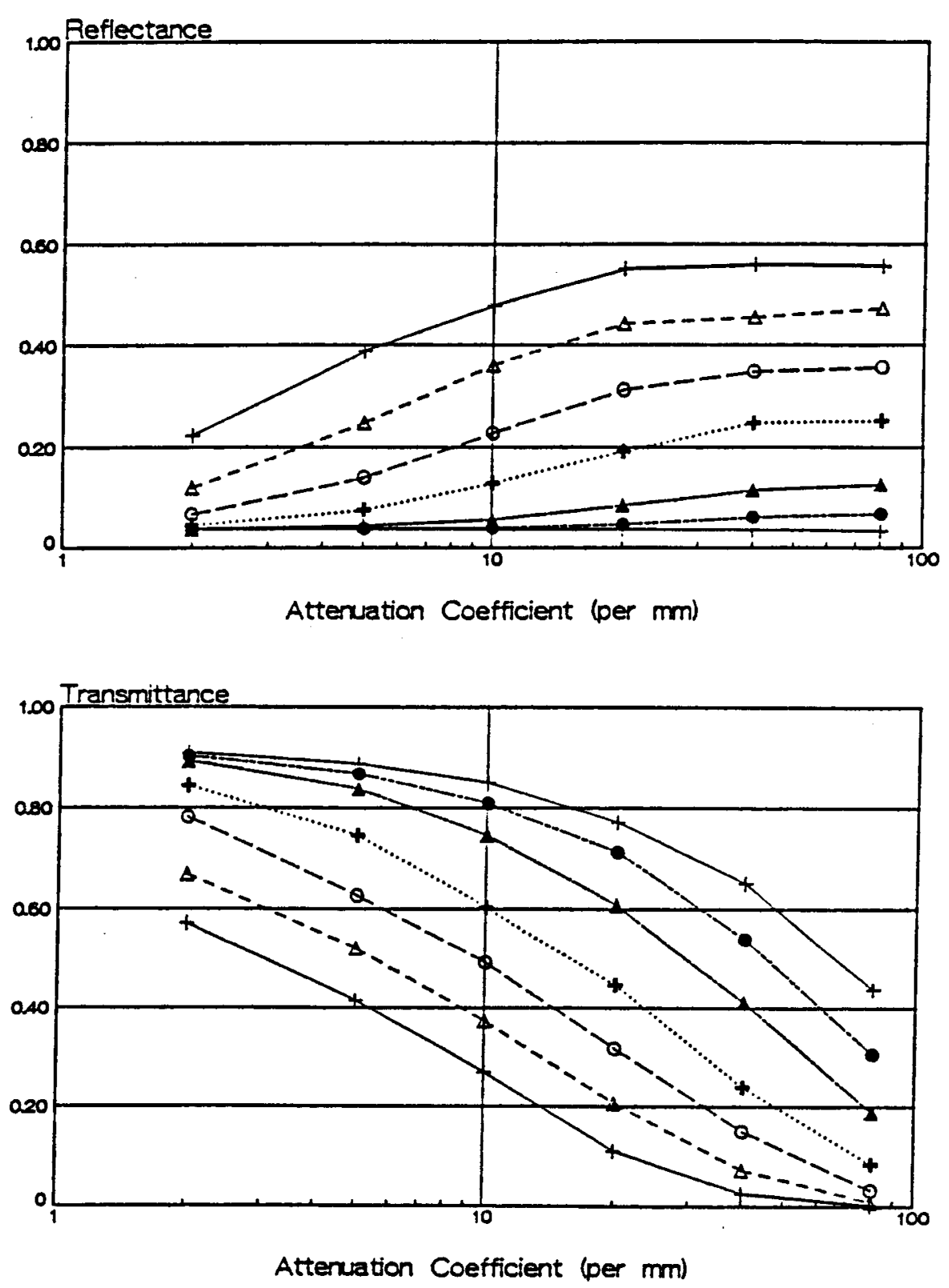

Figure 2.4 (continued)

C) Albedo $=0.995$ 
mean cosine of the scattering angle $=$ $+.60-\Delta-.80-0.90 \cdots+\cdots .95 \rightarrow .98-.99-.995$
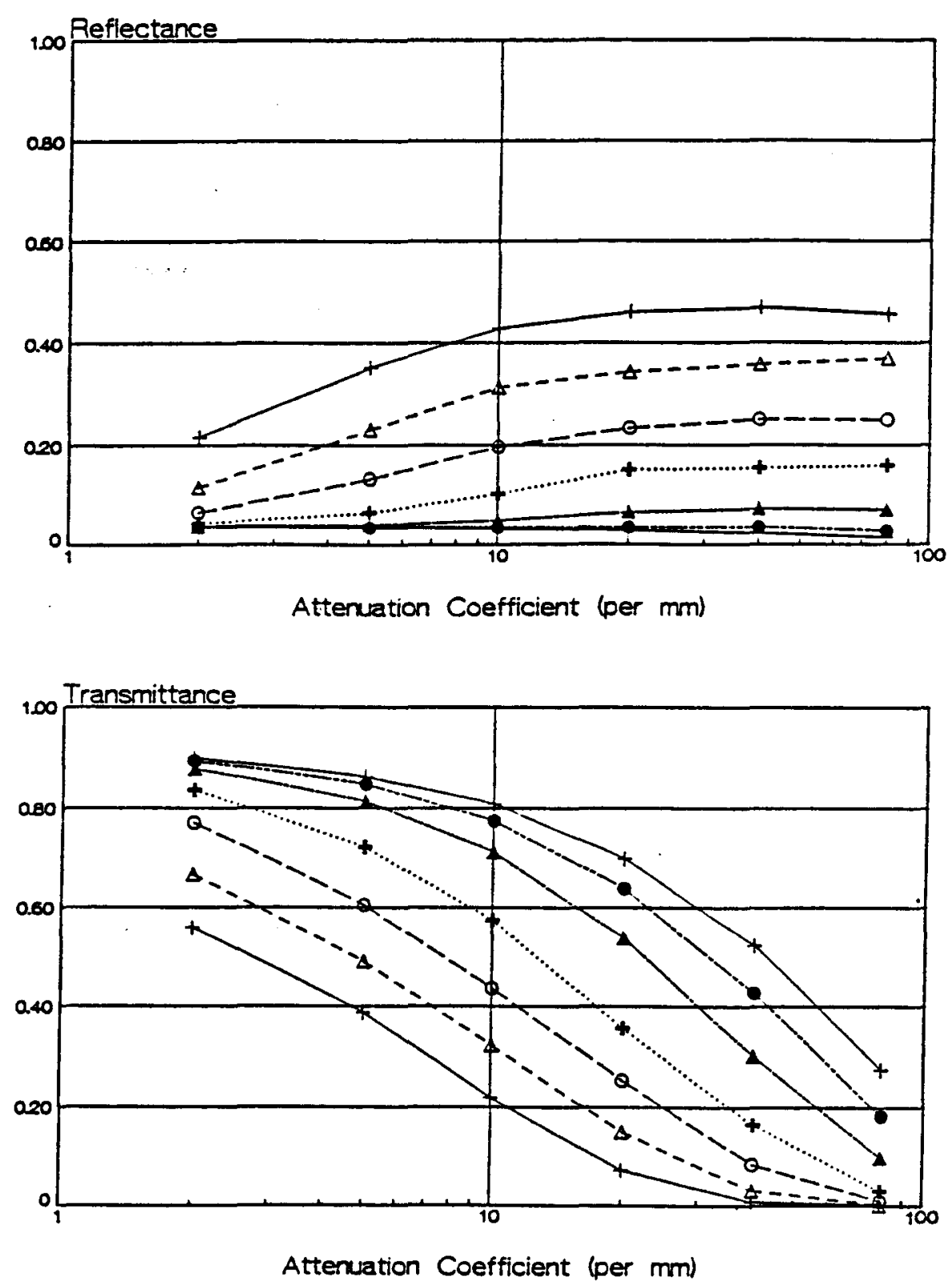

Figure 2.4 (continued)

d) Albedo $=0.990$ 
mean cosine of the scattering angle $=$

$+.60-\Delta-.80 \multimap . .90 \cdots+\cdots .95 \rightarrow .98 \rightarrow-.99+.995$

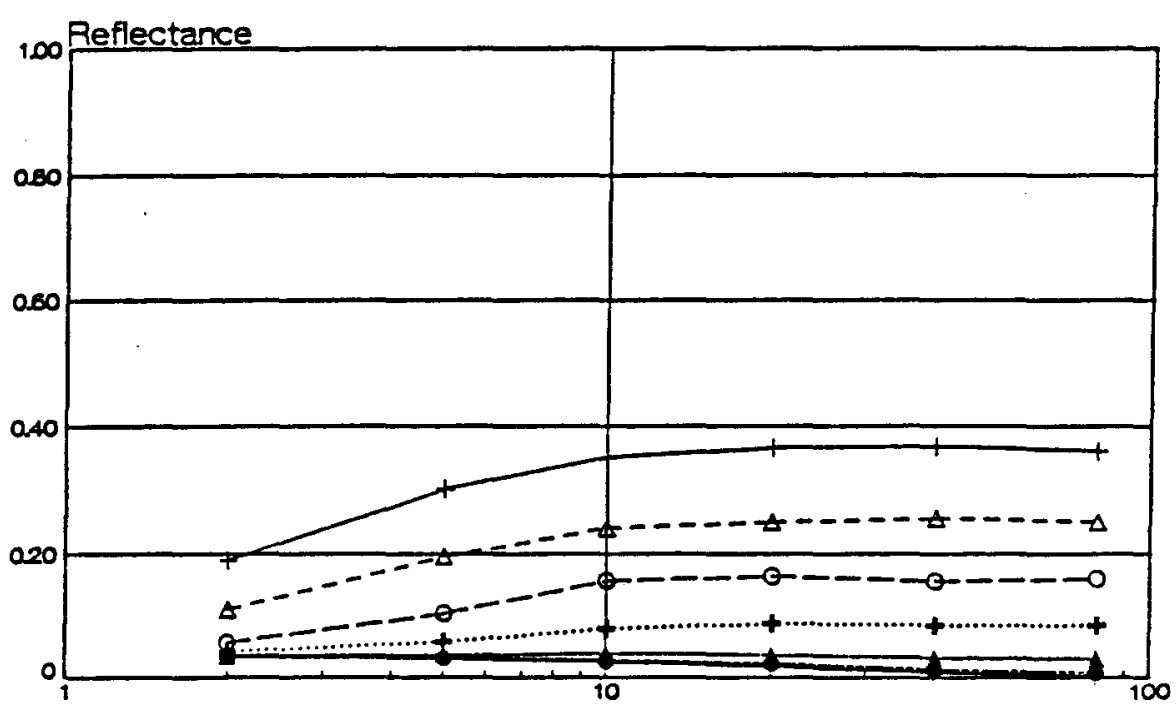

Attenuation Coefficient (per $\mathrm{mm}$ )

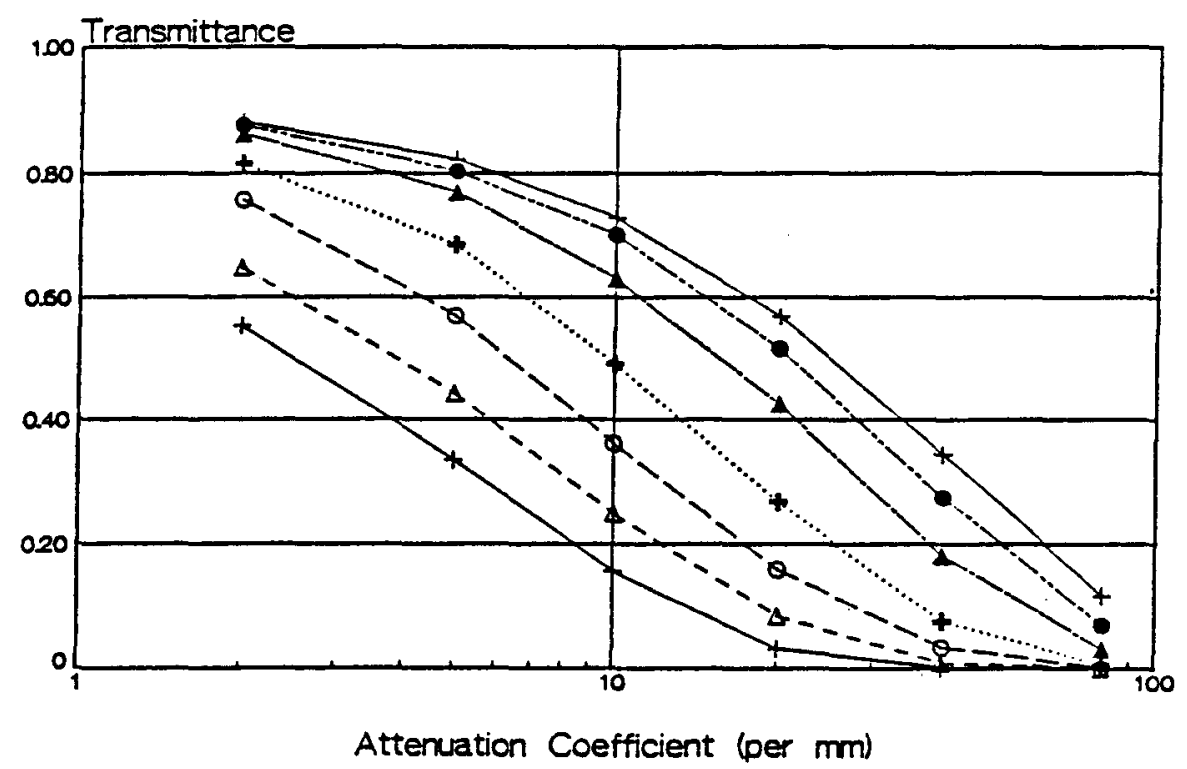

Figure 2.4 (continued)

e) Albedo $=0.980$ 
mean cosine of the scattering angle $=$

$+.60-\Delta-.80 \multimap-.90 \cdots+\cdots .95 \leftarrow .98-.99+.995$

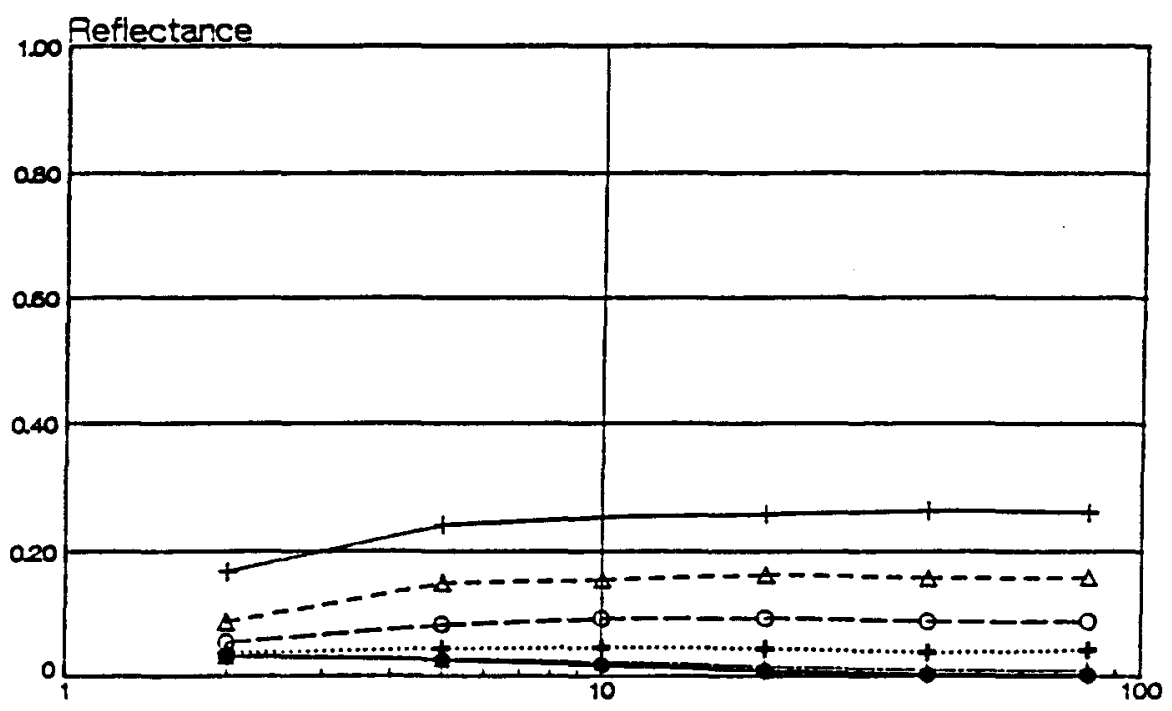

Attenuation Coefficient (per mm)

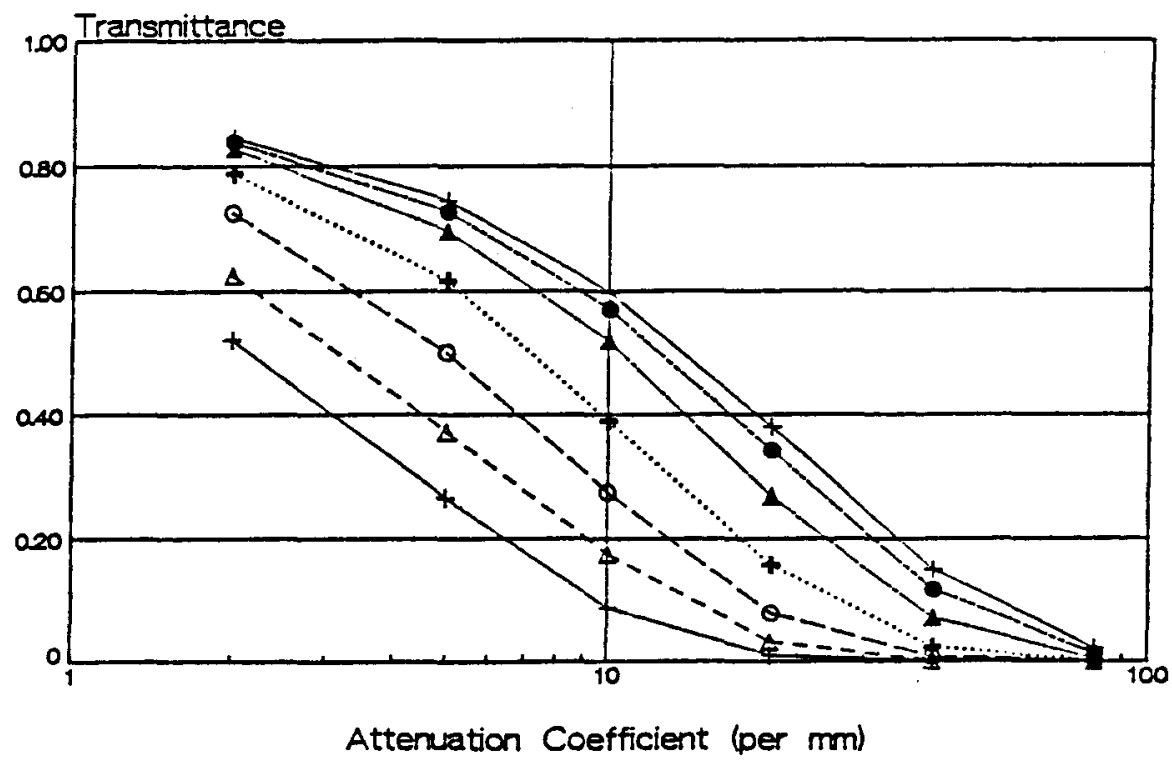

Eigure 2.4 (continued)

f) Albedo $=0.960$ 
Each Monte Carlo run consisted of 5000 incident photons divided into five groups of 1000 photons each. The errors in the calculated reflectance and transmittance were taken as the standard deviation in the mean of the five groups. The absolute errors in $R_{c}$ and $T_{c}$ are usually within $+/-0.005$ and are always within $+/-0.01$.

Systematic errors may be introduced through inappropriate selection of the indices of refraction. A difference of 0.07 in the index of refraction of tissue will result in errors in $R_{c}$ and $T_{c}$ which are usually within $+/-0.015$ but may be as large as $t /-0.03$. These limits were determined from Monte Carlo calculations for a range of expected values for $a, g$, and $\Sigma_{+}$. As will be shown in chapter 4 , these systematic errors are within the random errors for a given tissue sample. 


\section{CHAPTER 3}

\section{MATERIALS AND METHODS}

\subsection{Apparatus}

The light source used for these experiments was a high pressure xenon arc lamp (model Al000, Photon Technology International Inc., Princeton, New Jersey). The lamp housing incorporates a high efficiency light collection system using an ellipsoidal mirror, which focuses the light at a distance of $285 \mathrm{~mm}$ beyond the exit window. The typical spectral emission of a xenon lamp is reproduced in figure 3.1. The light was focused onto the entrance slit of a monochromator (model 01-001, PTI Inc.), which contains a reflection grating ruled at 1200 lines/mm. The monochromator was equipped with a stepping motor to permit computer control of the selected wavelength. The optical system, as shown in figure 3.2 , was designed to produce a well collimated, monochromatic light beam. A short focal length lens was positioned near the exit slit of the monochromator, producing a sharply focused, divergent beam. A chopper (model SR540, Stanford Research Systems Inc., Palo Alto, California) was placed in the beam to modulate the light signal. As will be discussed below, the light detection system employed a lock-in 


\section{Spectral Emission Curve}

Spectral Emission for Xenon Compact Arc Lamps
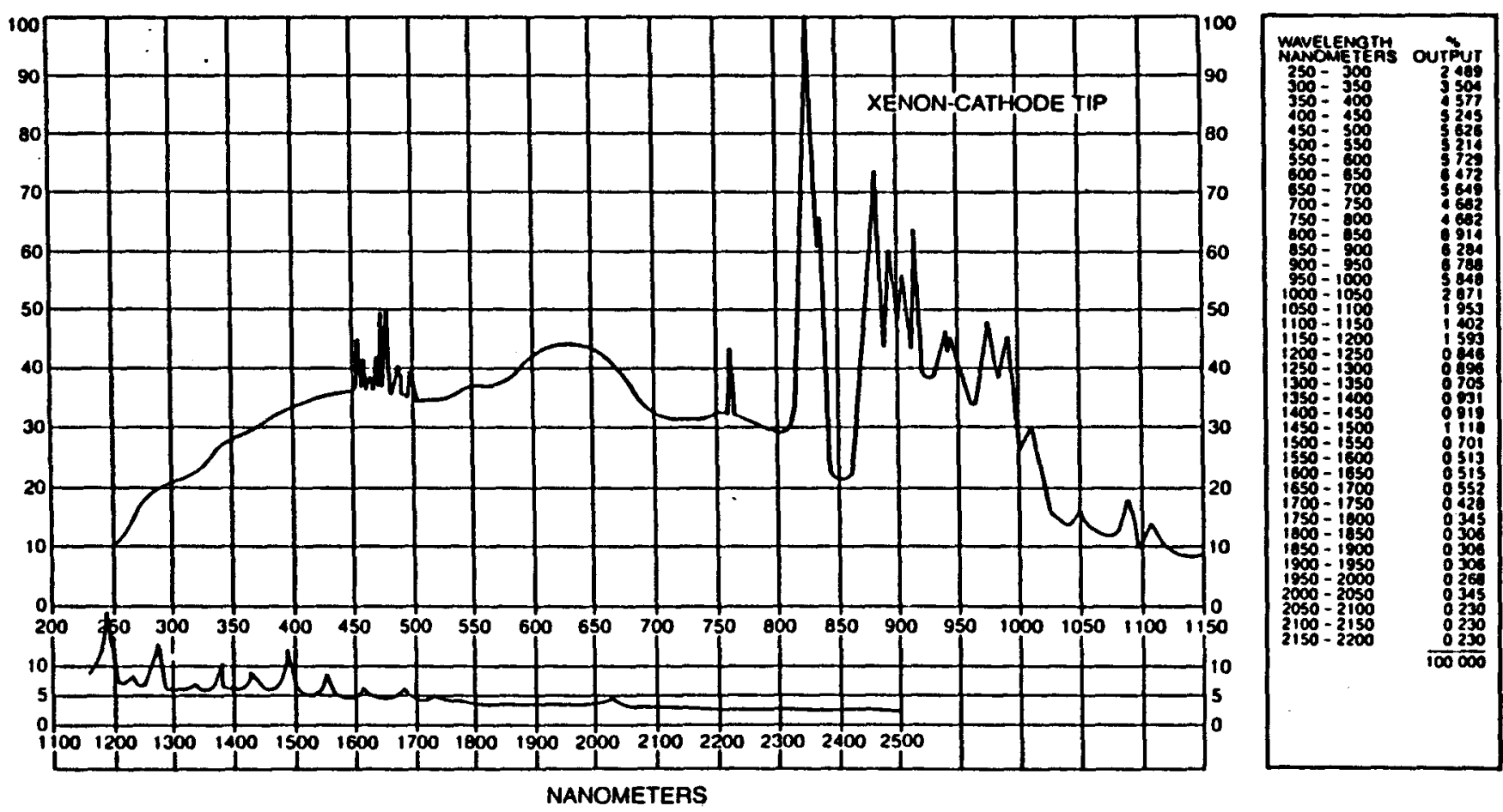

Figure 3.1 Typical spectral emission curve for a xenon arc lamp. 


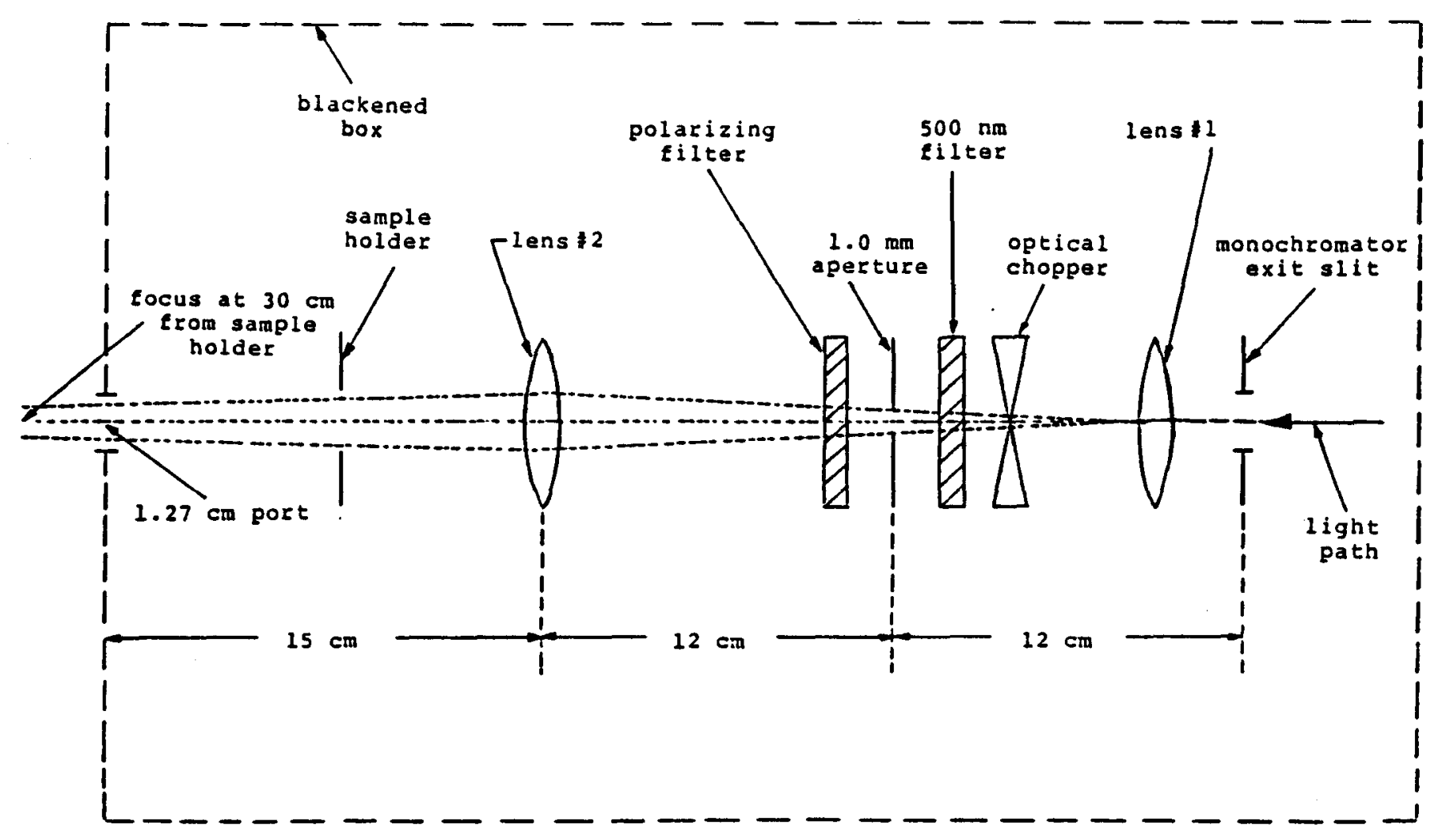

Figure 3.2 Optical system used to produce a collimated, monochromatic light beam. 
amplifier (model SR510, SRS Inc.) which selectively amplifies the modulated signal.

An optical filter was included to remove all wavelengths below approximately $500 \mathrm{~nm}$. This was done to avoid interference from second order diffraction lines passed through the monochromator. For some of the measurements a polarizing filter was inserted into the beam. A $1.0 \mathrm{~mm}$ aperture and a second lens were included to produce a narrow beam, focused at a distance of $37 \mathrm{~cm}$ beyond the lens. The optical system was enclosed in a blackened box to minimize interference by internal reflections and external light sources.

Silicon photodiodes (series S1336 BK, Hamamatsu Corp., Middlesex, New Jersey) were used as light detectors. The sensitivity of these detectors, as shown in figure 3.3 , is satisfactory for the range of wavelengths considered in this project. In figure 3.3 , the radiant sensitivity is defined as the diode output in amps (A), divided by the radiant energy incident on the device in watts (W). Since all experimental data are normalized to reference measurements at the same wavelength, the variations in spectral response are not important except when noise becomes a problem. When operated in the photovoltaic mode, the response is linear with respect to light intensity over approximately eight orders of magnitude. 


\section{Spectral Response}

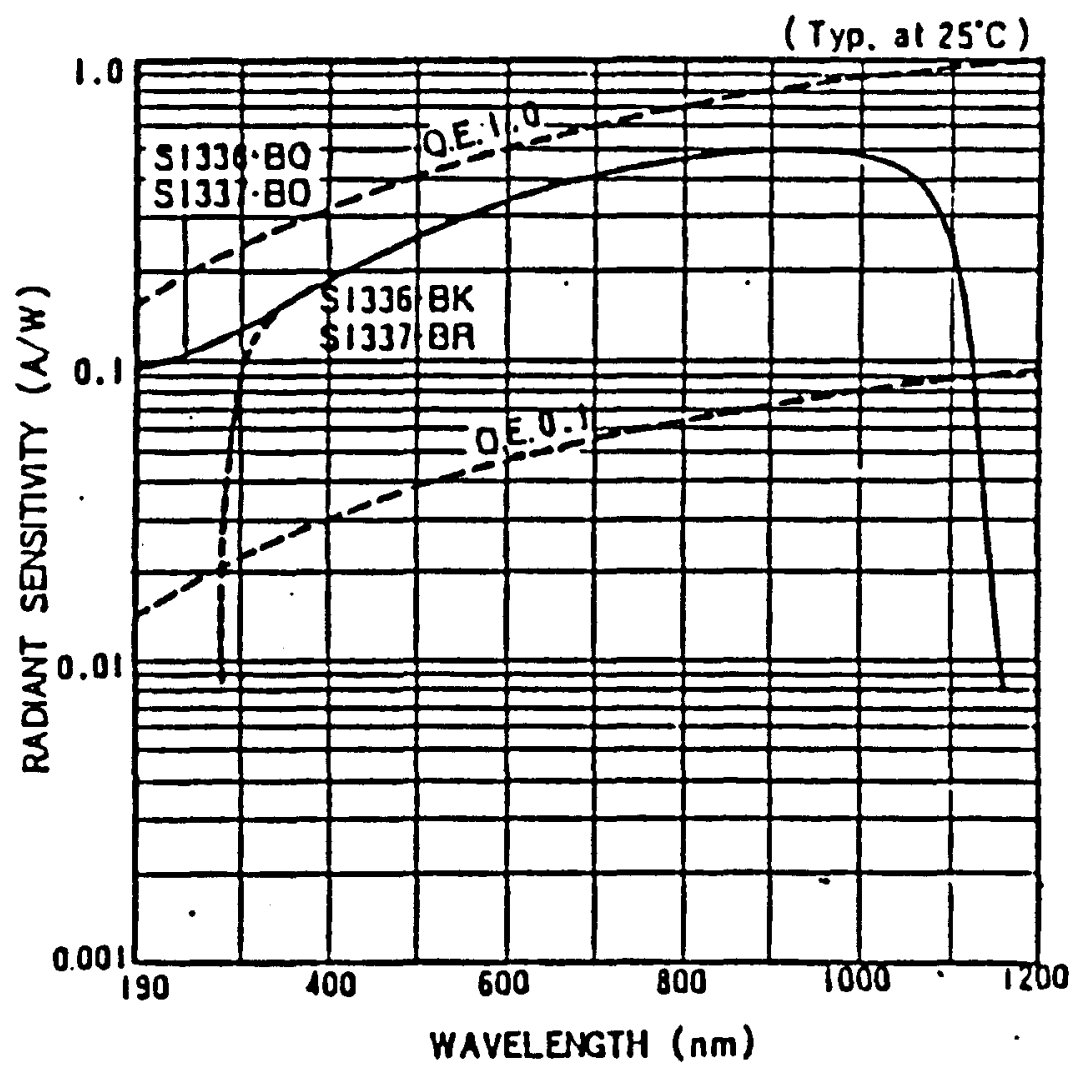

Figure 3.3 Radiant sensitivity of silicon photodiodes shown as the solid curve (manufacturers data). 
For measurement of the total attenuation coefficients, the light beam was directly incident on a collimated photodiode, coupled to a preamplifier (figure 3.4). The samples were positioned at a distance of $30 \mathrm{~cm}$ in front of the photodiode. This distance, combined with the use of a $0.2 \mathrm{~mm}$ aperture over the diode, was designed to minimize the amount of scattered light reaching the detector, while maintaining a good signal to noise ratio. Light scattered at angles greater than 0.02 degrees is rejected in this geometry. Further scatter rejection was achieved through use of a pair of polarizing filters. One filter was positioned on the source side of the sample and the other, over the detector. The first filter polarized the light incident on the sample; the second polarizer was oriented to transmit all photons with the same polarization direction. Light scattered in the sample will undergo a change in polarization and will, therefore, be preferentially attenuated by the second filter.

For the measurement of diffuse reflectance and transmittance, the photodiode was mounted on the inner surface of an integrating sphere (Labsphere Inc., North Sutton, New Hampshire). The sphere was $10.2 \mathrm{~cm}$ in diameter and included two opposing beam ports, $2.54 \mathrm{~cm}$ and $1.27 \mathrm{~cm}$ in diameter. Samples were always placed in contact with the sphere, at the smaller port. A third port, $1.27 \mathrm{~cm}$ in diameter and located at 90 degrees to the other ports, was 


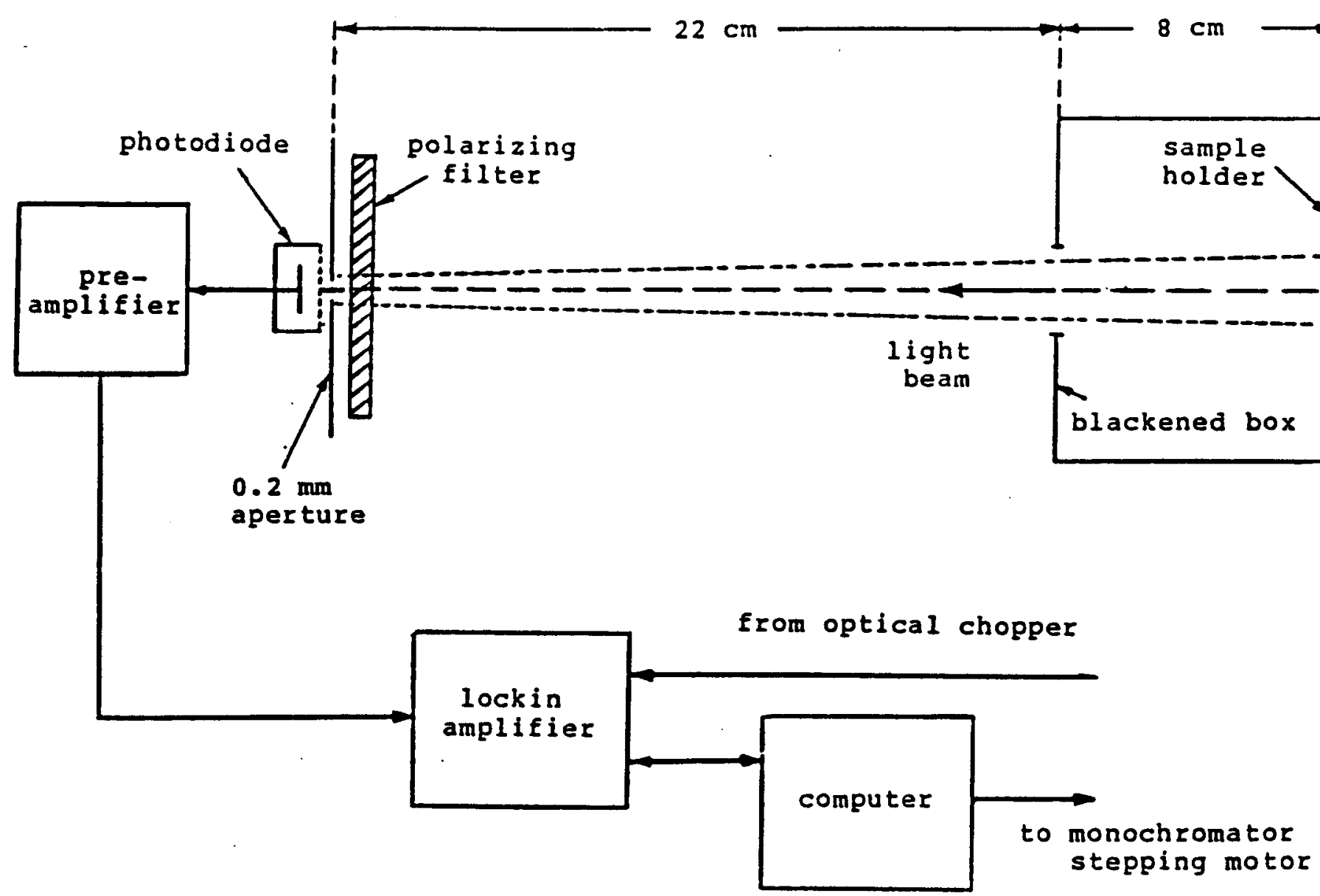

Figure 3.4 Experimental setup for measurement of the total attenuation coefficients. 
used to mount the photodiode detector. A light baffle was included between the detector port and the sample port to ensure multiple reflections within the sphere before the photons were detected. All inner surfaces of the sphere were coated with barium sulfate which causes the light to be diffusely reflected. This coating has a high refectivity which is nearly constant over the range of wavelengths used in these experiments (figure 3.5 ).

The geometries for measuring reflectance and transmittance are shown in figure 3.6. The centre of the sphere is positioned at the focus of the light beam emerging from the optical system. For reflectance measurements, the light beam enters the sphere through a $1.27 \mathrm{~cm}$ aperture placed over the $2.54 \mathrm{~cm}$ beam port. The inner surface of this aperture is coated with barium sulfate. For transmittance measurements, the sphere is rotated 180 degrees, and the $2.54 \mathrm{~cm}$ port is covered with a barium sulfate reflective plate. The beam size, at both the entrance and exit ports, was $3.0 \mathrm{~mm}$ in diameter. This ensured that the same area of the sample was illuminated for measurement of reflectance and transmittance.

For both detector geometries, the signal from the photodiodes was amplified by a lock-in amplifier. Essentially, this amplifier filters the signal and amplifies only those components which are modulated at the frequency of the chopper, thus improving the signal to 


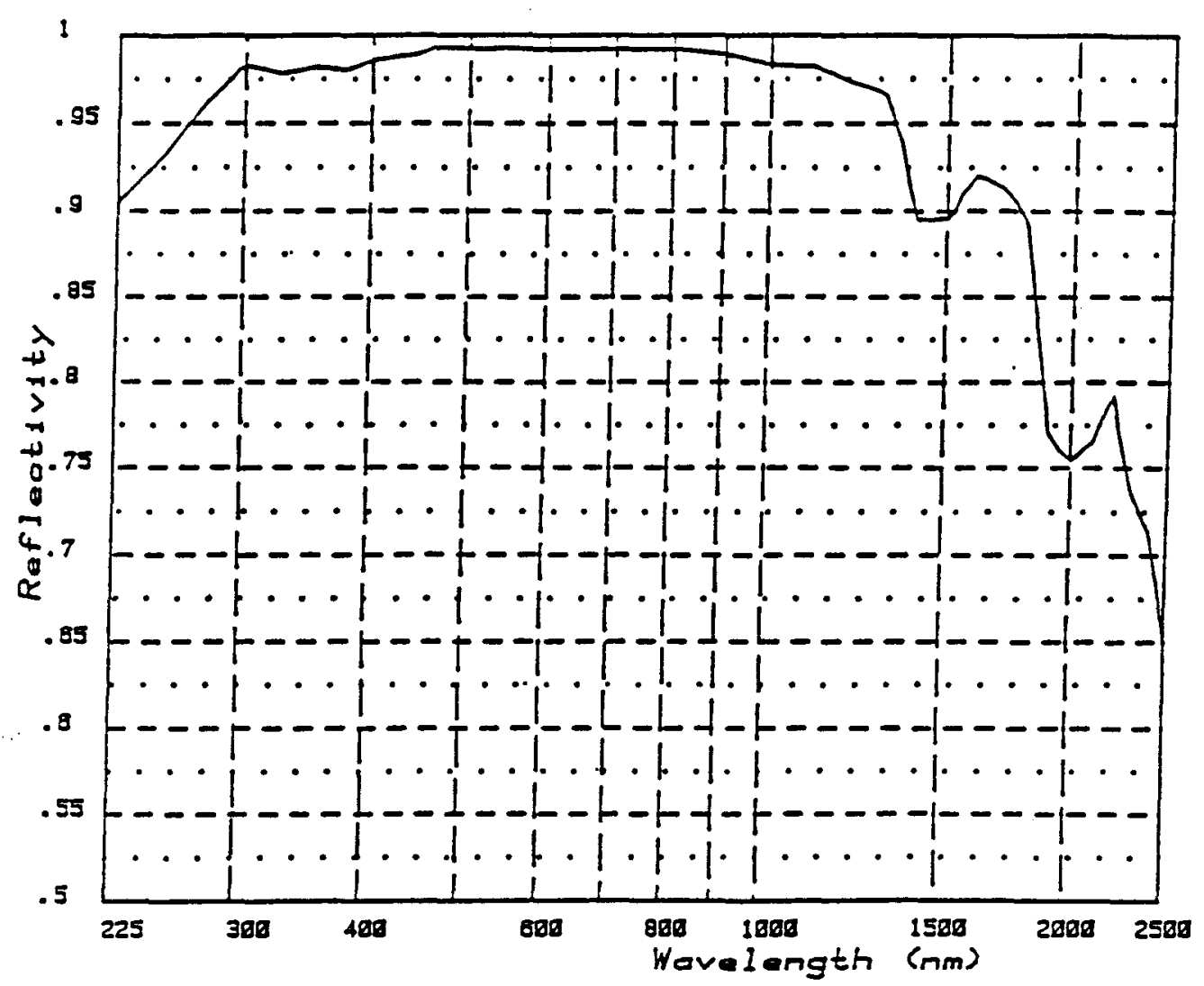

Eigure 3.5 Reflectivity of barium sulfate as a function of wavelength (Labsphere Inc., product data). 
a)

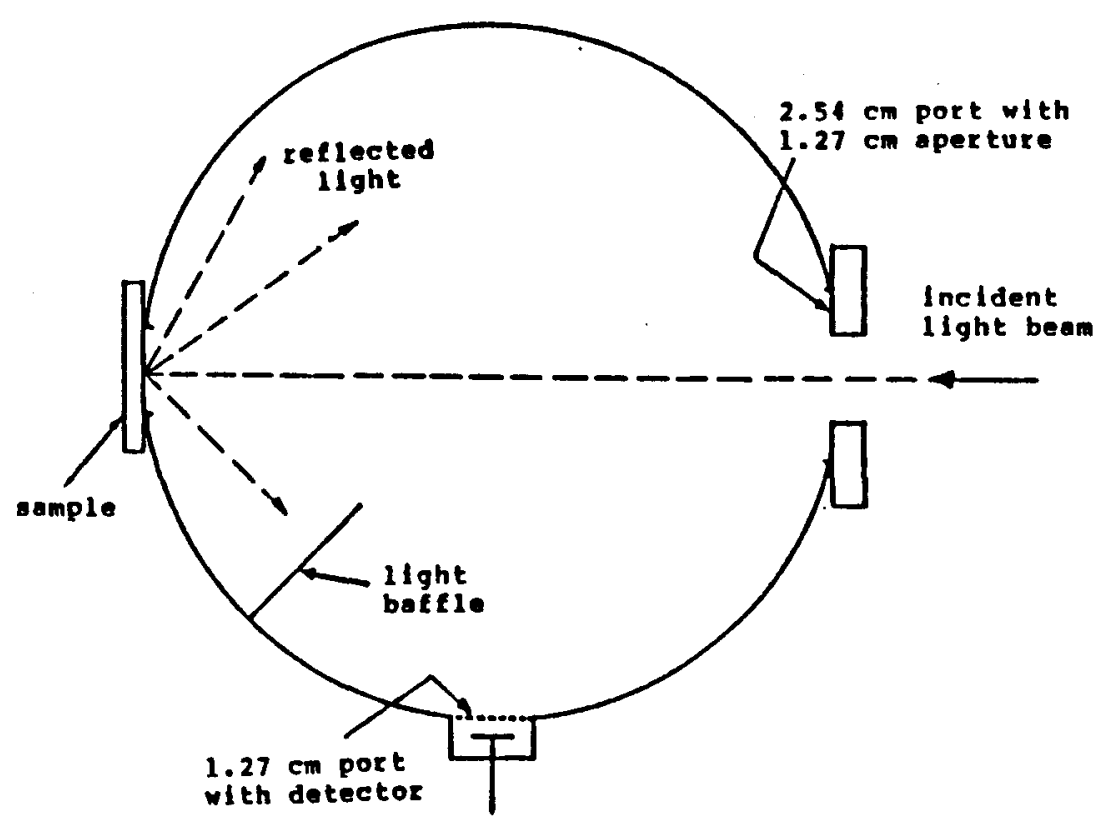

b)

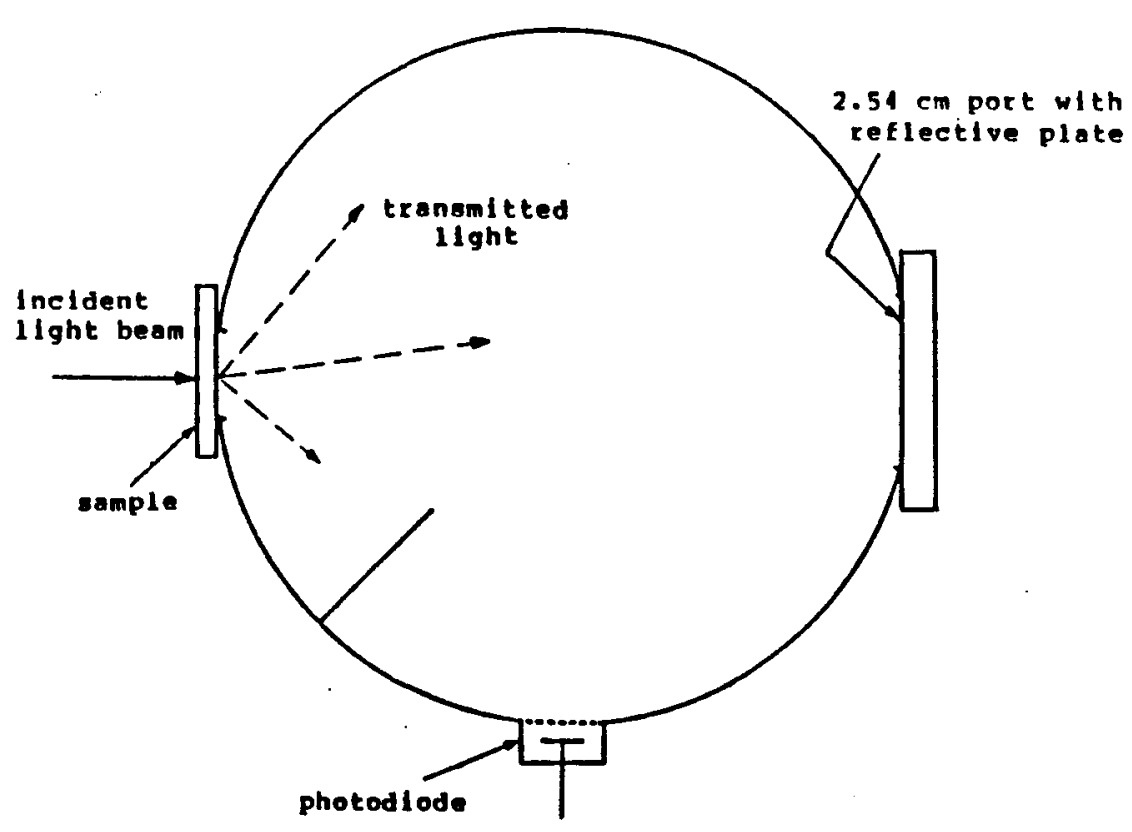

Figure 3.6 Integrating sphere geometries for the measurement of a) reflectance and b) transmittance. 
noise ratio. The chopper frequency was selected in such a way that background noise (e.g. room lighting, 60 cycle line frequency, etc.) was suppressed. A frequency of $80 \mathrm{~Hz}$ was found to be satisfactory.

Both the lock-in amplifier and the monochromator were interfaced to an IBM personal computer to permit computerized data acquisition and control. The computer code is provided in appendix $A$. The computer controls the stepping motor, which drives the monochromator to the selected wavelength; the computer then reads the signal from the amplifier. Sample measurements usually consisted of an automated scan over the range of wavelengths from 500 to $1100 \mathrm{~nm}$ at $10 \mathrm{~nm}$ increments.

\subsection{Calibration of the lamp and monochromator} The output stability of the lamp was tested at 633 $\mathrm{nm}$, using the setup shown in figure 3.4. During the first 2 hours after startup the output increased by approximately 10 percent, after which it remained stable within $+/-1.5$ percent. For all subsequent experiments, the lamp was allowed a 2 hour warmup period. Output checks were performed both before and after sample measurements, to ensure stability.

Since the output of the xenon lamp contains sharply peaked emission lines, the reproducibility of the monochromator wavelength setting may contribute significant 
errors when comparing the results of separate data sets. When using the stepping motor, the wavelength settings were always repeatable within $0.25 \mathrm{~nm}$. For the wavelength settings normally used, the measured optical output was repeatable within $+/-2.0$ percent.

The spectrum of the beam, measured at $2 \mathrm{~nm}$ increments using the photodiode, is shown in figure 3.7. The measured spectrum differs from that in figure 3.1, since the relative intensities have been modified by the spectral responses of the monochromator, photodiode, filter and lenses. However, a comparison of the positions of the principle emission lines verifies the calibration of the monochromator within $4 \mathrm{~nm}$. The resolution of the system, as given by the full width at half maximum of the sharpest of these lines, is approximately $4 \mathrm{~nm}$. The actual resolution may be better than indicated, since the lines in the xenon spectrum are expected to have some intrinsic width.

A further check of the calibration and resolution was performed using a helium-neon laser (633 nm). The laser beam was directed into the entrance slit of the monochromator and measurements made using a photodiode, as above. The maximum output intensity was observed at a monochromator setting of $633+/-1 \mathrm{~nm}$. The variation in this reading was dependent on the alignment of the beam on the entrance slit. The full width at half maximum was observed to be $2 \mathrm{~nm}$. This resolution may not be indicative 


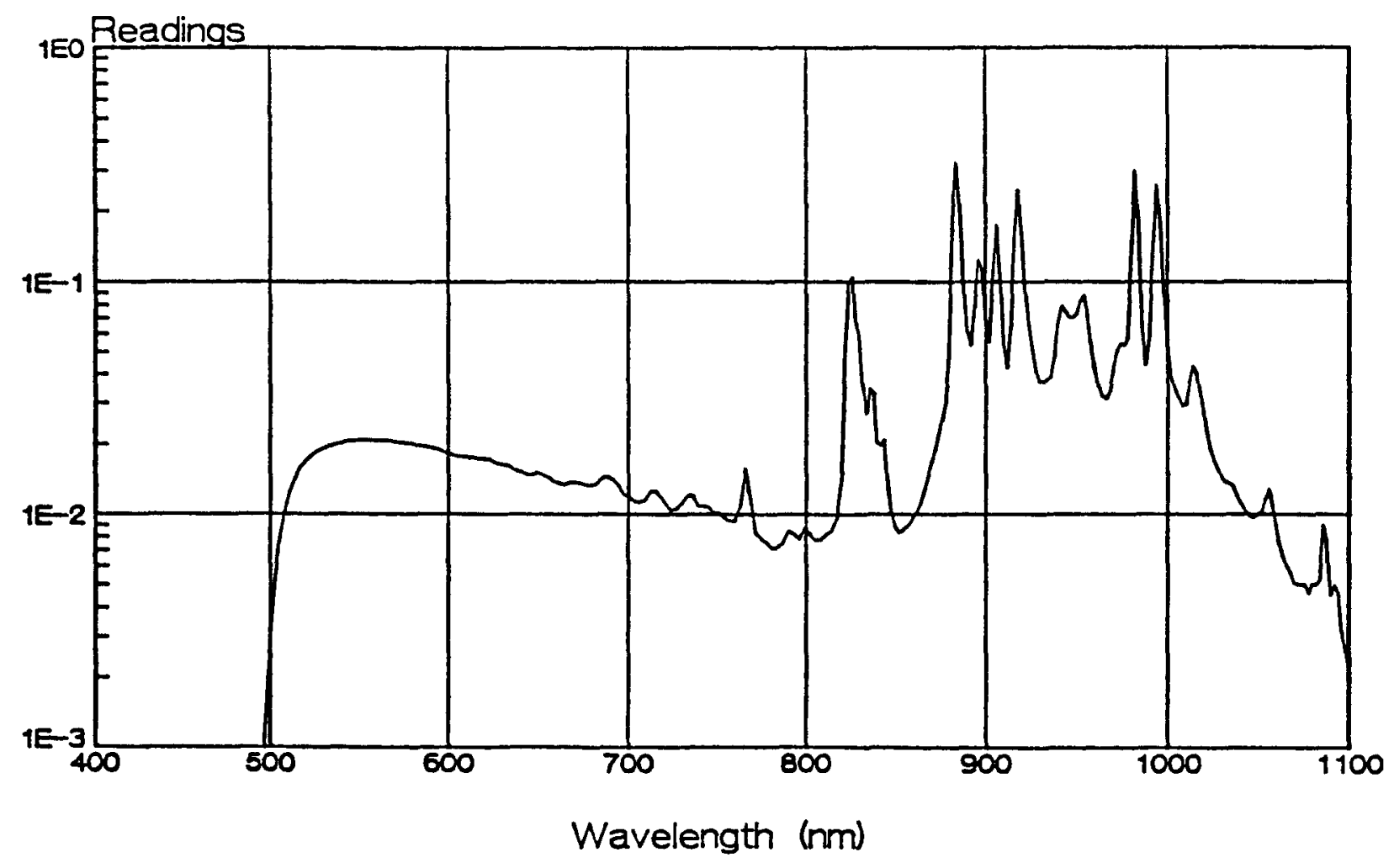

Figure 3.7 Measured spectrum of the light beam. 
of that which is attainable for the xenon lamp, since light from the lamp is focused on the entrance slit with a larger range of incident angles.

The reflectance geometry, as shown in figure $3.6(a)$, was used to conduct an additional wavelength calibration of the system with an erbium oxide standard (Labsphere Inc.). The calibration of this standard, with nine absorption peaks over the range 500 to $1000 \mathrm{~nm}$, is traceable to the National Bureau of Standards in the United States. The standard was placed at the sample port and measurements made over this wavelength range. Similar measurements were made with a barium sulfate reflection plate at the sample port. The ratio of these measurements was used to determine the positions of the absorption peaks (figure 3.8 ). This calibration indicates an accuracy within $3 \mathrm{~nm}$.

\subsection{Calibration of the integrating sphere}

Using the measurement geometry of figure $3.6(a)$, the reflectance of a sample may be determined from

$$
R=R_{s}\left(I_{R} / I_{s}\right) \quad 3.1
$$

where $I_{R}$ is the reflectance measurement with the sample, $I_{s}$ is the reflectance measurement with a barium sulfate reflective plate in the sample position, and $R_{s}$ is the reflectivity of the barium sulfate sphere coating. 


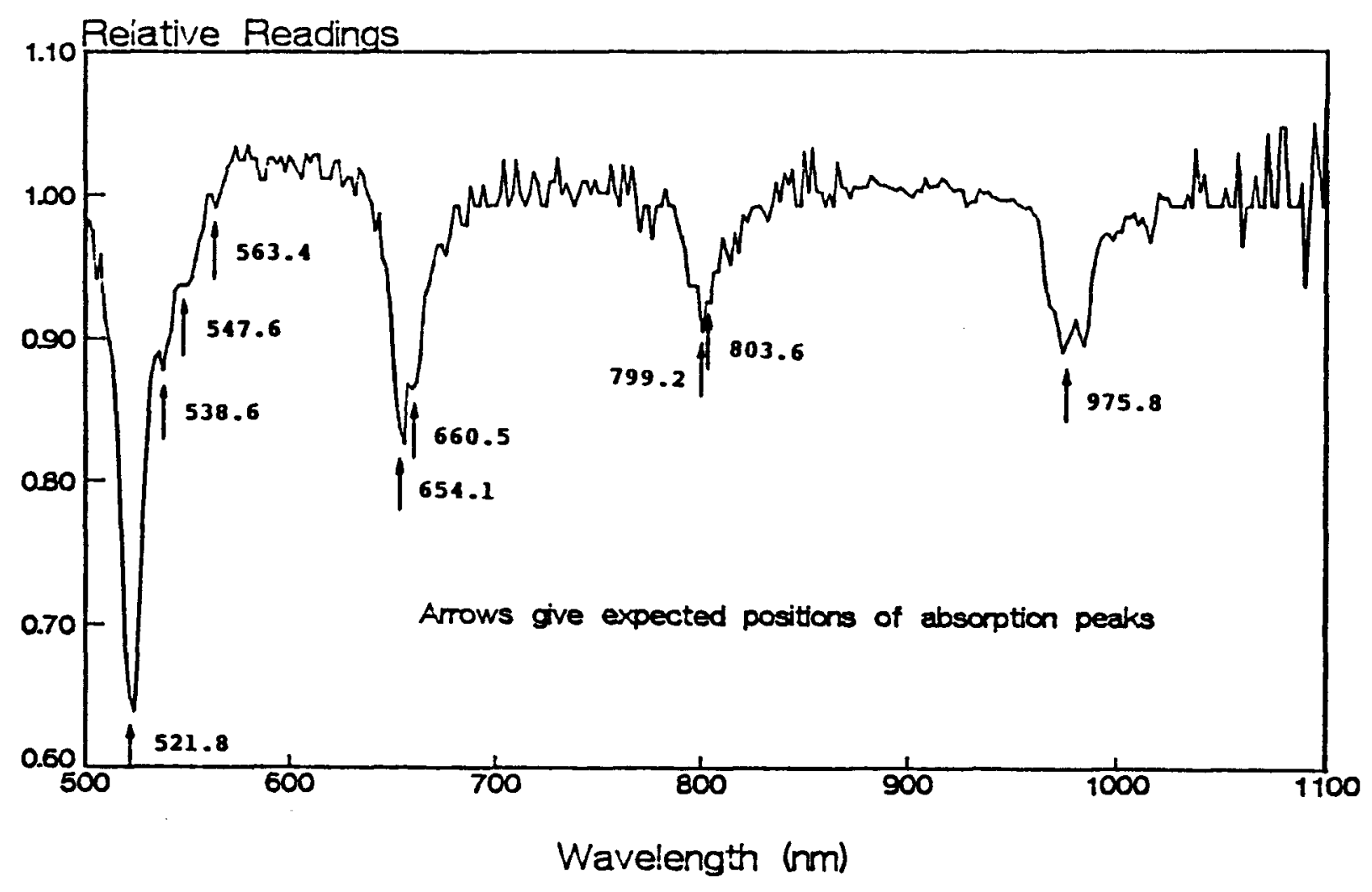

Eigure 3.8 Wavelength calibration of the monochromator, using an erbium oxide absorption standard. Reflectance readings with the standard are normalized to the readings obtained using a barium sulfate reflector. 
Similarly, the transmittance is given by

$$
\begin{aligned}
& T=R_{S}\left(I_{T} / I_{S}\right) \\
& \text { the setup shown in figure } 3.6(b),
\end{aligned}
$$

and $I_{s}$ and $R_{s}$ are the reflectance readings and reflectivity for barium sulfate as defined above. These formulae are derived in appendix $B$.

A set of reflectance standards (Labsphere, Inc.) was available to test the validity of the reflectance formula, as well as to determine the value of $R_{s}$. These standards, with nominal reflectance values of $0.99,0.50$, 0.20 and 0.05 , were calibrated by the manufacturer using reference materials obtained from the National Bureau of Standards in the United States. The stated error in the calibrated values was less than 0.005. In turn, each reflectance standard was placed against the sample port of the integrating sphere in the geometry of figure $3.6(a)$. Measurements were taken at $50 \mathrm{~nm}$ increments over the range 500 to $1100 \mathrm{~nm}$. Similar measurements were taken for a barium sulfate plate.

For each wavelength, the reflectance of the barium sulfate coating was calculated from

$$
R_{5}=R_{50}+\left(R_{99}-R_{50}\right) *\left[\left(I_{5}-I_{50}\right) /\left(I_{99}-I_{50}\right)\right] 3.3
$$

where $R_{99}$ and $R_{50}$ were the calibrated reflectivities of the reflectance standards, having nominal reflectivities of 0.99 and 0.50 , respectively. $I_{99}$ and $I_{50}$ were the 
readings obtained with these reflectance standards. This method assumes Iinearity of response over this range of reflectances. The reflectivity of the sphere coating was determined to be $0.965+/-0.01$ over the entire wavelength range of interest. This value is approximately 2 percent lower than that provided by the manufacturer (figure 3.4). Figure 3.4, however, applies to pure barium sulfate and does not include the effect of binders, which are added to provide adhesion to the surfaces (Labsphere Inc., product data sheet).

A sphere reflectivity of 0.965 was used in formula 3.1, to calculate the reflectance of each standard at all wavelengths where measurements were made. The results were compared with the calibrated reflectance and are plotted in figure 3.9. For clarity, individual points have not been plotted. The range of values for each reflectance standard are plotted as a rectangle. The width of each rectangle is determined by the range of calibrated reflectance values over all wavelengths; the height is determined by the range of reflectance values as calculated using equation 3.1 . The absolute error is within approximately 0.03 at a reflectivity of 0.50 , and improves at both lower and higher reflectivities.

Since the same sources of error are inherent in the development of the transmittance equation, the accuracy of this value is also expected to be within 0.03 . 


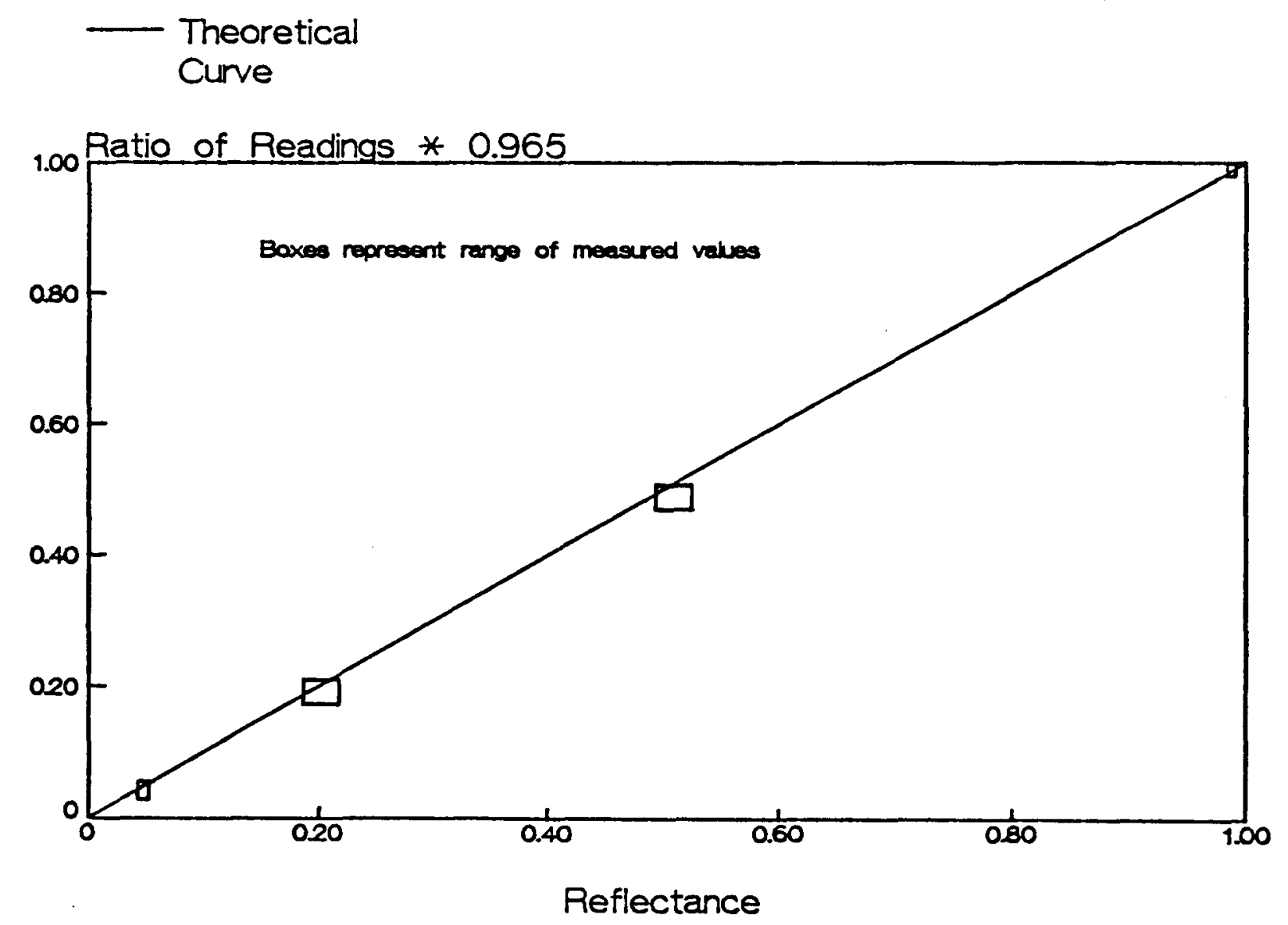

Figure 3.9 Calibration of the integrating sphere using four reflectance standards. The calculated reflectance is plotted as a function of the calibrated reflectance. Each box encloses the range of values obtained for wavelengths from 500 to $1100 \mathrm{~nm}$. 
Equations 3.1 and 3.2 were applied in the analysis of all sample results. Corrections to the reflectance based on the measurements with the calibration standards (figure 3.9) were not applied, since similar correction factors for the transmittance were unavailable. Intrasample inhomogeneities in tissues were shown to result in differences in $R$ and $T$ which equal or exceed these systematic errors.

For a sample held between glass slides, the calculated reflectance and transmittance values will correspond to the restricted definitions used in section 2.3.3 for the purposes of Monte Carlo modeling. These definitions include the effects of specular reflections at glass-air interfaces, absorption of light in glass, and boundary conditions at the entrance and exit surfaces of the slides. To distinguish these quantities from the actual sample reflectance and transmittance, they will be denoted by $R_{M}$ and $T_{M}$.

\subsection{Reflectance and transmittance measurements}

Freshly excised breast tissues were received from the pathology department of the Henderson General Hospital (Hamilton, Ontario). These specimens were stored in closed jars and kept frozen at -25 degrees Celsius to prevent deterioration. The effects of freezing were investigated by comparing the results of measurements using fresh tissues 
with those of frozen tissues from the same specimen.

As has already been discussed, the samples were held between $1.0 \mathrm{~mm}$ thick glass slides. Since some of the tissues received from pathology had volumes of less than $0.1 \mathrm{~cm}^{3}$, it was decided to use a sample thickness of 1.0 mm. The lateral dimensions of the prepared samples were about $1.0 \times 1.0 \mathrm{~cm}$. Due to the difficulty of slicing samples of uniform thickness, it was decided to homogenize the tissues by 'dicing' them with a scalpel. This technique also assured greater uniformity throughout the prepared samples. The homogenized tissues were lightly compressed between the glass slides using a $1.0 \mathrm{~mm}$ spacer to ensure a uniform thickness. The edges of the slides were sealed with epoxy adhesive to prevent dehydration of the sample. The validity of the homogenization technique was tested by comparing the results using homogenized samples with those of $1.0 \mathrm{~mm}$ thick slices cut from the same specimen. Reflectance and transmittance measurements were performed using the integrating sphere, as shown in figure 3.6. Automated scans were performed, with readings being taken at $10 \mathrm{~nm}$ increments over the range 500 to $1100 \mathrm{~nm}$. Similar measurements were always made with the barium sulfate plate in the reflectance geometry. The sample reflectance, $R_{M}$, and transmittance, $T_{M}$, were then calculated according to equations 3.1 and 3.2 . 


\subsection{Total attenuation measurements}

In principle, the measurement of the total attenuation coefficients, using the experimental arrangement of figure 3.4 , is relatively simple. The attenuation coefficient of the tissue is given by

$$
\Sigma_{+}=-(1 /+) \ln \left(I / I_{0}\right)
$$

where $I$ is the detector reading with the sample,

$I_{0}$ is a reference reading with a water sample,

and $t$ is the thickness of the tissue sample.

The water sample was constructed using the same type of glass slides as used for the tissue samples and is, therefore, subject to the same specular reflection losses and absorption in glass. The thickness of the water sample was less than $0.004 \mathrm{~mm}$ to minimize absorption in water. For tissues, the attenuation coefficients are expected to lie in the range $10 \mathrm{~mm}^{-1}$ to $100 \mathrm{~mm}^{-1}$ (Flock et al, 1987). Therefore, the attenuation of light passing through a $1.0 \mathrm{~mm}$ thick sample would be from 5 to 45 orders of magnitude. In addition, the beam emerging from such a sample would certainly contain forward directed photons, which have been multiply scattered. For these reasons, a thinner sample is required.

It was decided to use a microtome to cut thin frozen sections of tissue for measurement. These tissues were taken from the same specimens used to prepare the 1.0 $\mathrm{mm}$ thick samples. The cut slices were mounted between glass 
slides using a small amount of water to provide better coupling between the tissues and glass by eliminating air spaces. The edges of the slides were then sealed with epoxy. For each specimen, slices were cut at several thicknesses ranging between 0.004 and $0.024 \mathrm{~mm}$, as determined by the microtome setting.

For adipose tissue, it was not possible to cut slices using the microtome. Homogenization, as used in preparing the $1.0 \mathrm{~mm}$ samples, was also ineffective in producing uniform samples of less than about $0.1 \mathrm{~mm}$. The only acceptable method for preparing sections was to cut thin slices from a frozen specimen using a scalpel. The slices were mounted between glass slides and compressed slightly, while still frozen, to obtain a uniform thickness. Careful compression was necessary to avoid the rupture of cells and loss of cellular fluids. Several samples were prepared from each specimen, with thicknesses ranging between 0.03 and $0.20 \mathrm{~mm}$. The thicknesses were determined using a micrometer and are accurate to within about $+/-0.005 \mathrm{~mm}$.

Measurements were made for each sample using the setup shown in figure 3.4. An automated scan was performed over the wavelength range 500 to $1100 \mathrm{~nm}$. To minimize the effects of local inhomogeneities in the samples, an aperture was positioned over the sample holder. The aperture size was selected and each sample positioned such 
that a tissue area of reasonable uniformity was exposed to the primary beam. The typical aperture size was $0.4 \times 0.4$ $\mathrm{cm}$. The same aperture was subsequently used for the reference measurement with a water sample.

At each wavelength, the attenuation of the sample was calculated as

$$
-\ln \left(I / I_{0}\right)
$$

and the results for all slices taken from the same specimen were then plotted as a function of slice thickness. These points were fitted to a straight line and the attenuation coefficient determined from the slope of this line. This technique eliminates the error associated with surface scattering at the glass-tissue interface. According to Flock et al (1987), a linear fit is valid for sample thicknesses less than about $0.2 \mathrm{~mm}$. For thicker samples, detection of multiply scattered photons caused a deviation from linearity. The geometry used by Flock et al was less effective than ours in rejecting scatter, having a detector acceptance angle of 0.17 degrees.

The attenuation coefficient for glass was required as input to the Monte Carlo program. This was determined using

$$
\Sigma_{t}=-(1 / t) \ln \left[\left(I / I_{0}\right) \cdot\left(1+R_{\text {ag }}\right) /\left(1-R_{\text {ag }}\right)\right] \quad 3.6
$$

where $t$ is the thickness of the glass slide, $I$ is the reading with a glass slide in the sample holder (figure 3.4), 
$I_{0}$ is the reading with nothing in the sample holder, and $\boldsymbol{R}_{a g}$ is the specular reflectance at a single air-glass interface.

The factor $\left(1+R_{a g}\right) /\left(1-R_{a g}\right)$ corrects for specular reflection losses in a glass slab surrounded by air (Kortum, 1969). For normal incidence on glass, having a refractive index of $1.5, R_{a g}$ has a value of 0.04 .

\section{6 optical properties of Intralipid}

Intralipid (Kabivitrum Inc., Dorval, Quebec), a fat emulsion, has been used as a phantom material to simulate the light scattering properties of tissues. The optical properties of Intralipid, containing 1.0 percent solids, were derived using the methods already described. The reflectance and transmittance were measured for a $1.0 \mathrm{~mm}$ thick sample contained in a glass cuvette with $1.0 \mathrm{~mm}$ thick walls. The same cuvette was used for measurement of the total attenuation coefficient, with varying dilutions of Intralipid ( 0.2 to 1.0 percent solids) used to provide different optical thicknesses. The values of the albedo and the mean cosine of the scattering angle were determined using the Monte Carlo model described in section 2.3.3. These results were compared with other published values. 


\subsection{Verification of the mathematical model}

A suspension of polystyrene microspheres in water was used to test the validity of the Monte Carlo analysis. These microspheres, obtained from the University of Bristol (U.K.), are expected to cause light scattering with negligible absorption. The size of the spheres was 1003 $+/-32 \mathrm{~nm}$ and their refractive index was given as 1.57. The scattering coefficient is dependent on the concentration of spheres as well as their size. Mie theory (Bohren and Huffman, 1983) can be used to determine both the scattering coefficient and the mean cosine of the scattering angle. A computer program was written to determine the optical properties according to this theory. Calculations were performed for wavelengths over the range 500 to $1000 \mathrm{~nm}$. The optical properties of the spheres were also derived using the Monte Carlo method. The reflectance and transmittance were measured for a $1.0 \mathrm{~mm}$ thick suspension containing 0.95 percent spheres by volume. The sample was held in a glass cuvette with $1.0 \mathrm{~mm}$ thick walls. The same cuvette was used to measure the total attenuation coefficient using varying dilutions of spheres 10.04 to 0.20 percent spheres by volume). The albedo and the mean cosine of the scattering angle were then determined using the Monte Carlo technique described in section 2.3 .3 . 
To test the technique for different values of the absorption coefficient, varying amounts of india ink were added to the microsphere suspensions (maximum ink concentration of 0.1 percent). The reflectance and transmittance were measured for each suspension of spheres with added ink. The Monte Carlo technique was used to derive the albedo and the mean scattering angle for each suspension. For comparison, a direct measurement of the absorption coefficient was made, as described below. Since the absorption by polystyrene microspheres is expected to be very low, the absorption coefficient of a suspension of microspheres with added ink will equal the absorption coefficient for the ink alone with a small contribution from water. The absorption coefficients were, therefore, determined for water samples prepared using the same concentrations of ink. The measured reflectance of these water suspensions was zero, indicating that india ink is essentially a pure absorber. The absorption coefficient for each suspension of ink in water was, therefore, taken to be equal to the attenuation coefficient measured using the method described in section 3.5 . 


\section{CHAPTER 4}

\section{RESULTS AND DISCUSSION}

\subsection{Intralipid}

To illustrate the method of data analysis and presentation, the results for Intralipid will be described first.

The measured reflectance and transmittance for a suspension of Intralipid, containing 1.0 percent solids, are shown in figure 4.1. The uncertainty in each of these quantities is primarily due to the systematic errors previously discussed. The reflectance, $R_{M}$, falls smoothly as the wavelength increases, while the transmittance, $T_{M}$, increases. No significant fine detail is observed, except for a reduction in both $R_{m}$ and $T_{m}$ over a broad band centered at about $970 \mathrm{~nm}$. This reduction in the total 1 ight detected will be shown to represent absorption of light in the sample. It should be noted that $R_{m}+T_{m}$ will always be less than 1.0 , due to incomplete collection of the reflected and transmitted light.

Figure 4.2 shows attenuation coefficients, measured for four samples of Intralipid containing different concentrations of solids. For each sample, this coefficient falls smoothly as a function of increasing wavelength. 
- R

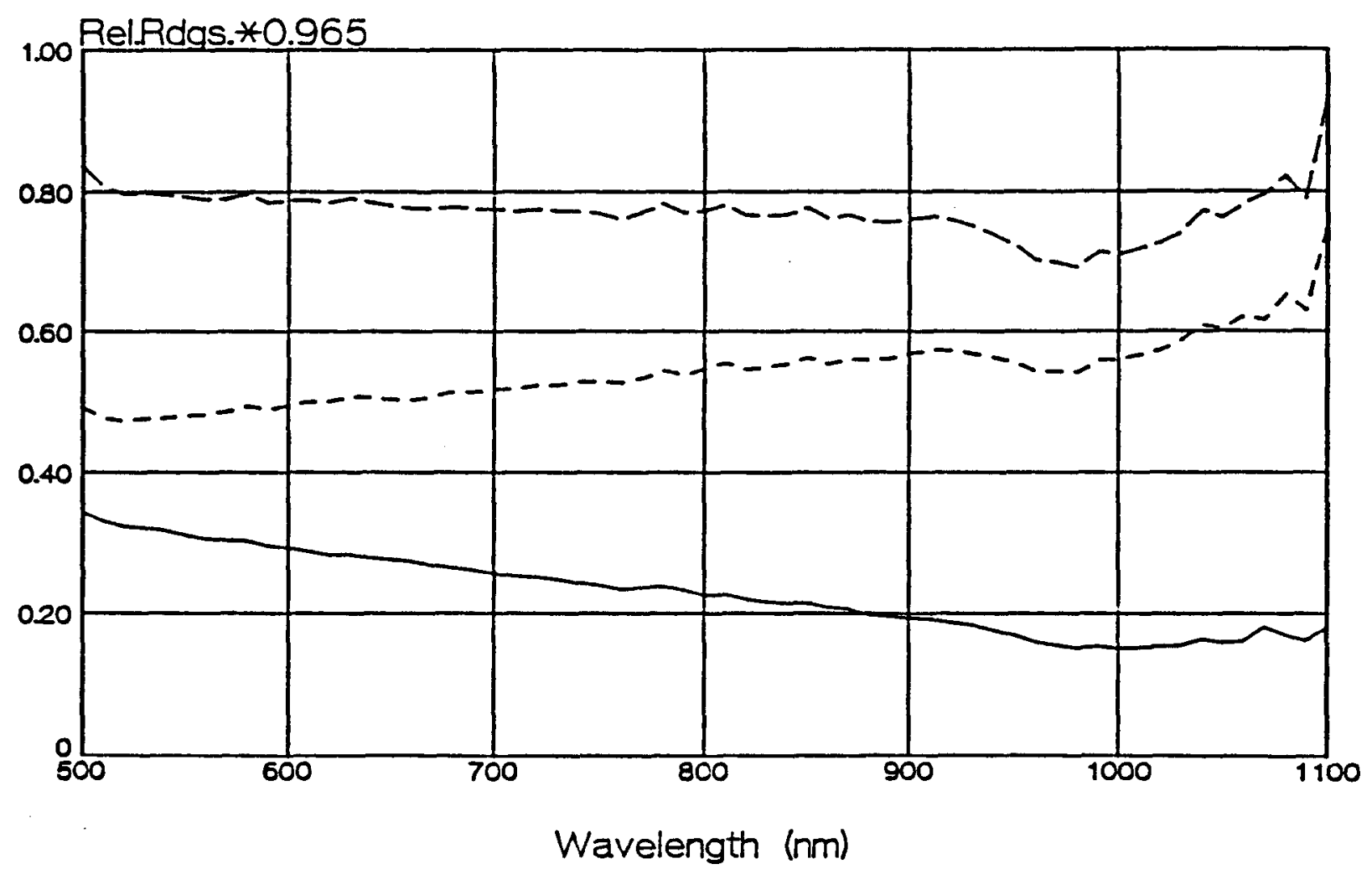

Figure 4.1 Measured reflectance (R) and transmittance (T) for a suspension of Intralipid containing 1.0 percent solids (sample thickness $=1.0 \mathrm{~mm}$ ). 


\begin{tabular}{|c|c|c|}
\hline $\begin{array}{l}0.2 \% \\
\text { solids }\end{array}$ & $\begin{array}{c}---0.4 \% \\
\text { solids }\end{array}$ & $\begin{array}{c}-0.6 \% \\
\text { solids }\end{array}$ \\
\hline
\end{tabular}

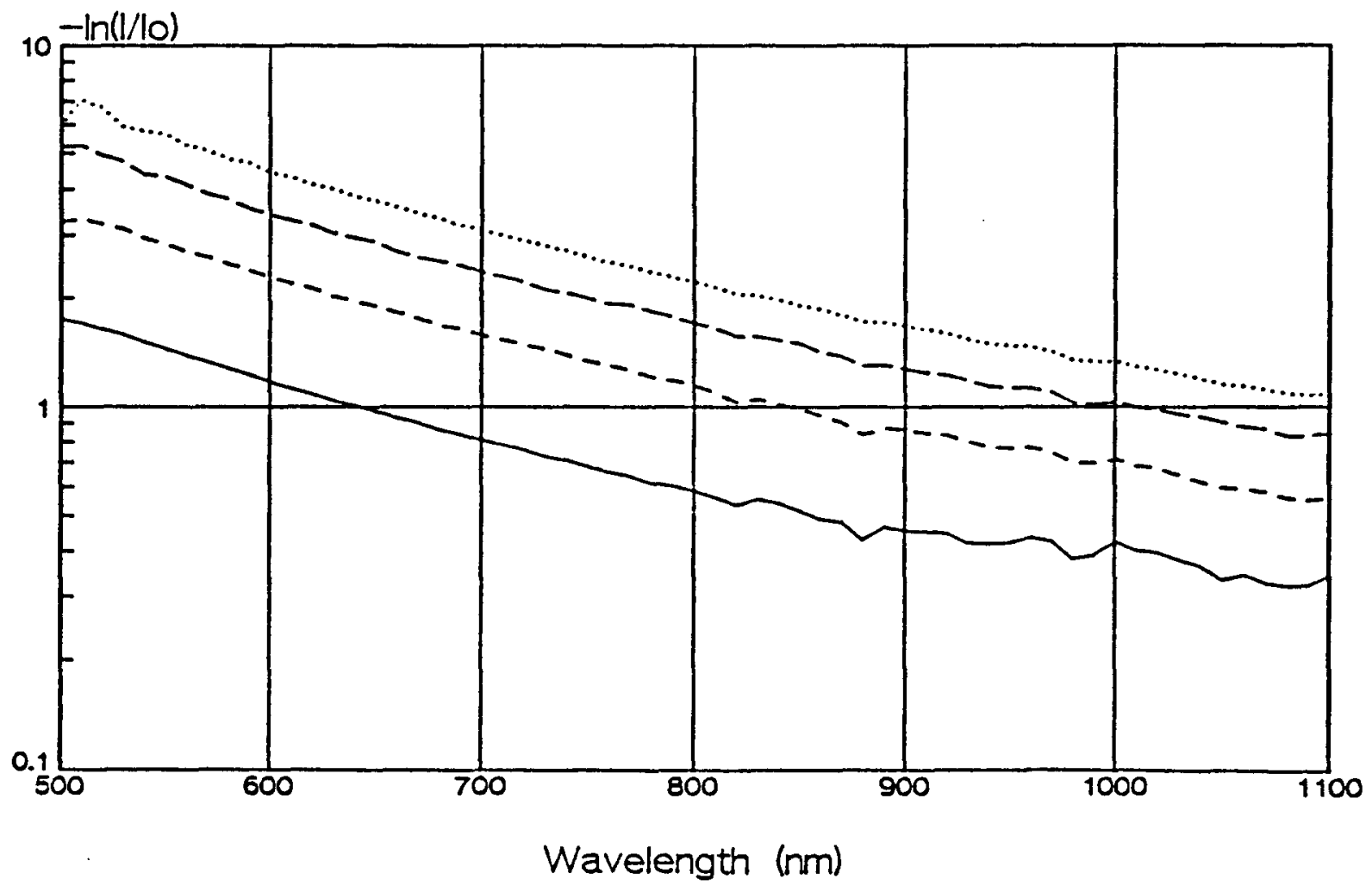

Figure 4.2 Measured total attenuation for suspensions of Intralipid containing four different concentrations of solids

(sample thickness $=1.0 \mathrm{~mm}$ ). 
Since the total attenuation is primarily due to the solid components rather than water, each concentration can be represented by an equivalent thickness. For selected wavelengths, the attenuation coefficients have been replotted as a function of the equivalent thickness in figure 4.3. The data was normalized such that a suspension containing 1.0 percent solids has a thickness of $1.0 \mathrm{~mm}$. The data points in figure 4.3 were fit by least squares regression lines. Since all points are well described by the linear fit, we can conclude that the detected signal contains few scattered photons. Further, since the lines intersect near the origin, we conclude that all light losses occur within the sample, with little interference from glass interfaces. The attenuation coefficient at each wavelength has been taken as the slope of the appropriate line. The standard deviation in this value, calculated using standard methods (Bevington, 1969), is less than 1.0 percent, except at $500 \mathrm{~nm}$. Figure 4.4 shows the attenuation coefficients replotted as a function of wavelength, decreasing smoothly from a value of $7.2 \mathrm{~mm}^{-1}$ at $500 \mathrm{~nm}$ to $1.2 \mathrm{~mm}^{-1}$ at $1100 \mathrm{~nm}$. The value of $4.8 \mathrm{~mm}^{-1}$ at $633 \mathrm{~nm}$ is in excellent agreement with that obtained by Hansen (1988) and Wilson et al (1986), but differs from the value of $3.84 \mathrm{~mm}^{-1}$ measured by Flock et al (1987). 

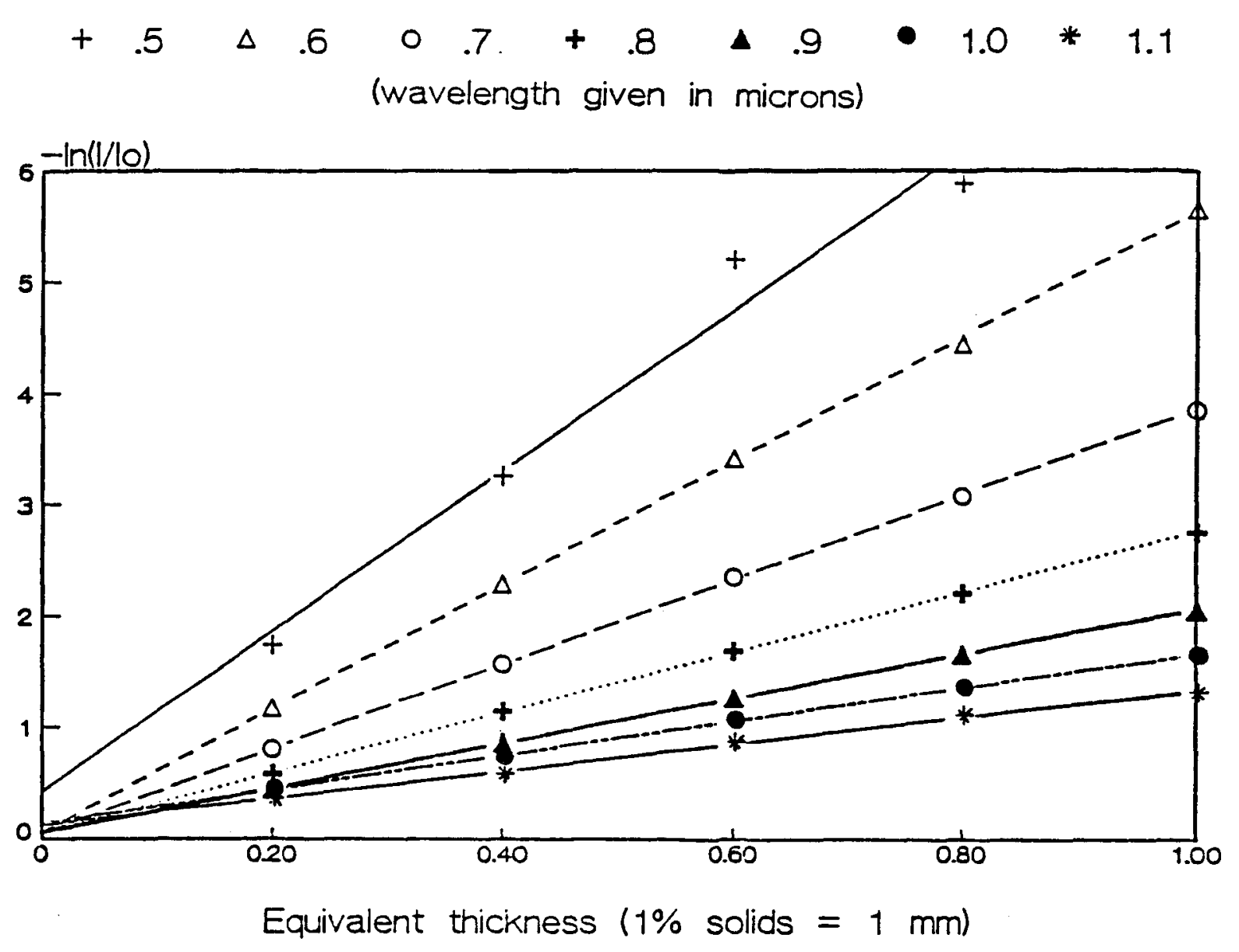

Eigure 4.3 plots of the total attenuation in Intralipid for selected light wavelengths. Varying concentrations of Intralipid have been converted to equivalent thickness assuming that a suspension containing 1.0 percent solids has a thickness of $1.0 \mathrm{~mm}$. 


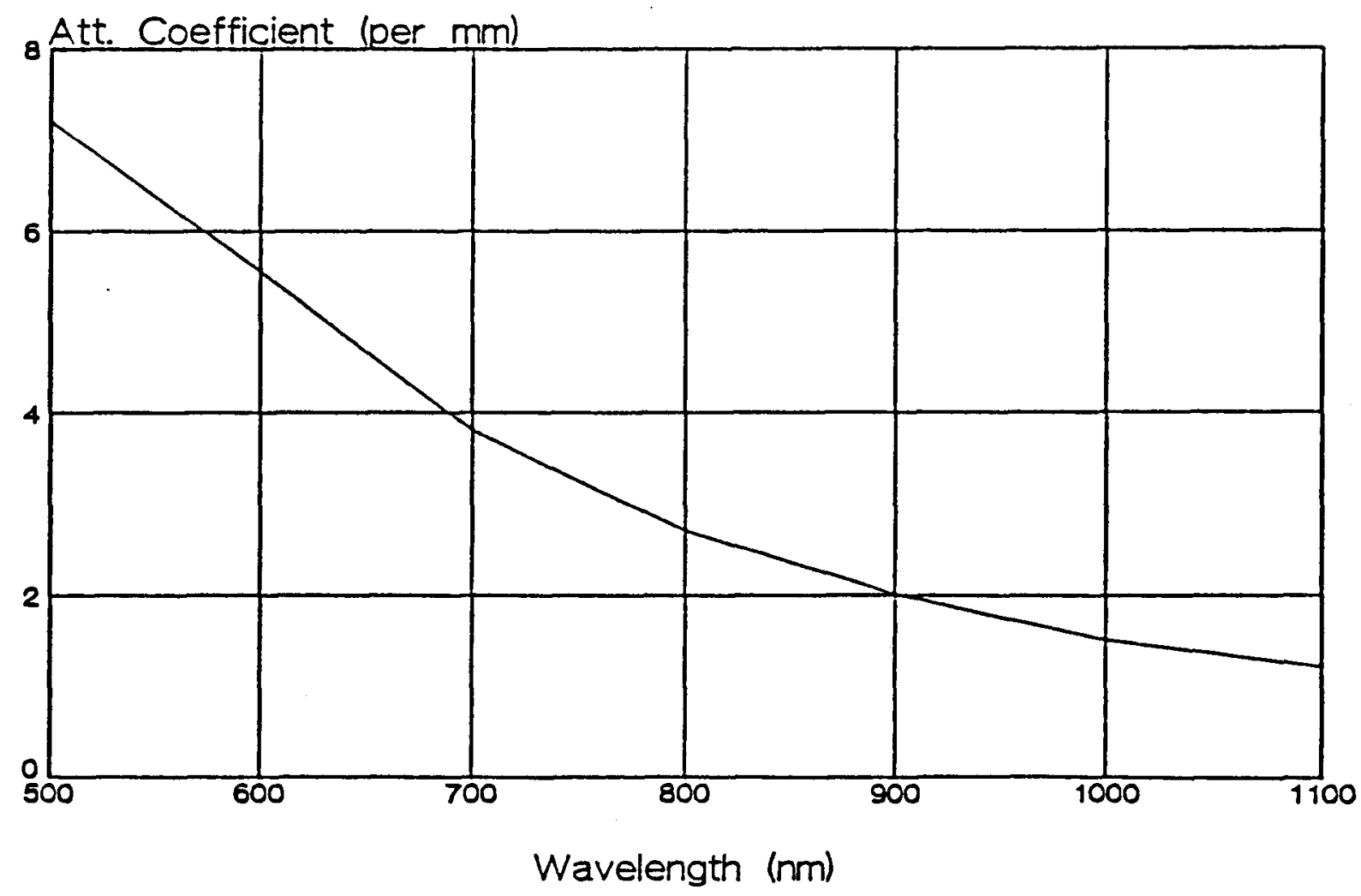

Figure 4.4 Total attenuation coefficients for a suspension of Intralipid containing 1.0 percent solids. This data was taken from the slope of the curves in figure 4.3 . 
The optical properties derived using the Monte Carlo model are shown in figure 4.5. Typical error bars are also indicated. These errors are determined by the range of solutions obtainable from the Monte Carlo simulations, based on the errors in the three measured quantities. The errors in $R_{m}$ and $T_{m}$ are taken to be $+/-0.03$. The derived absorption coefficient is about two orders of magnitude smaller than the scattering coefficient, and shows a broad absorption peak centered at $970 \mathrm{~nm}$. This coincides with the expected location and magnitude of a water absorption peak (Driscoll and Vaughan, 1978). The mean cosine of the scattering angle falls smoothly with increasing wavelength, from 0.80 at $500 \mathrm{~nm}$ to 0.54 at $1050 \mathrm{~nm}$. The value of 0.76 $+/-0.03$ at $633 \mathrm{~nm}$ differs from the value of 0.69 (no error given) obtained by Flock et al (1987). This discrepancy may be a result of differences in the composition of the phantom materials, or may indicate the inadequacy of the Henyey-Greenstein function in modeling the scatter of light.

\subsection{Polystyrene microspheres}

The reflectance and transmittance for the suspension of polystyrene microspheres are shown in figure 4.6. The total attenuation coefficients were determined using the method described for Intralipid. 


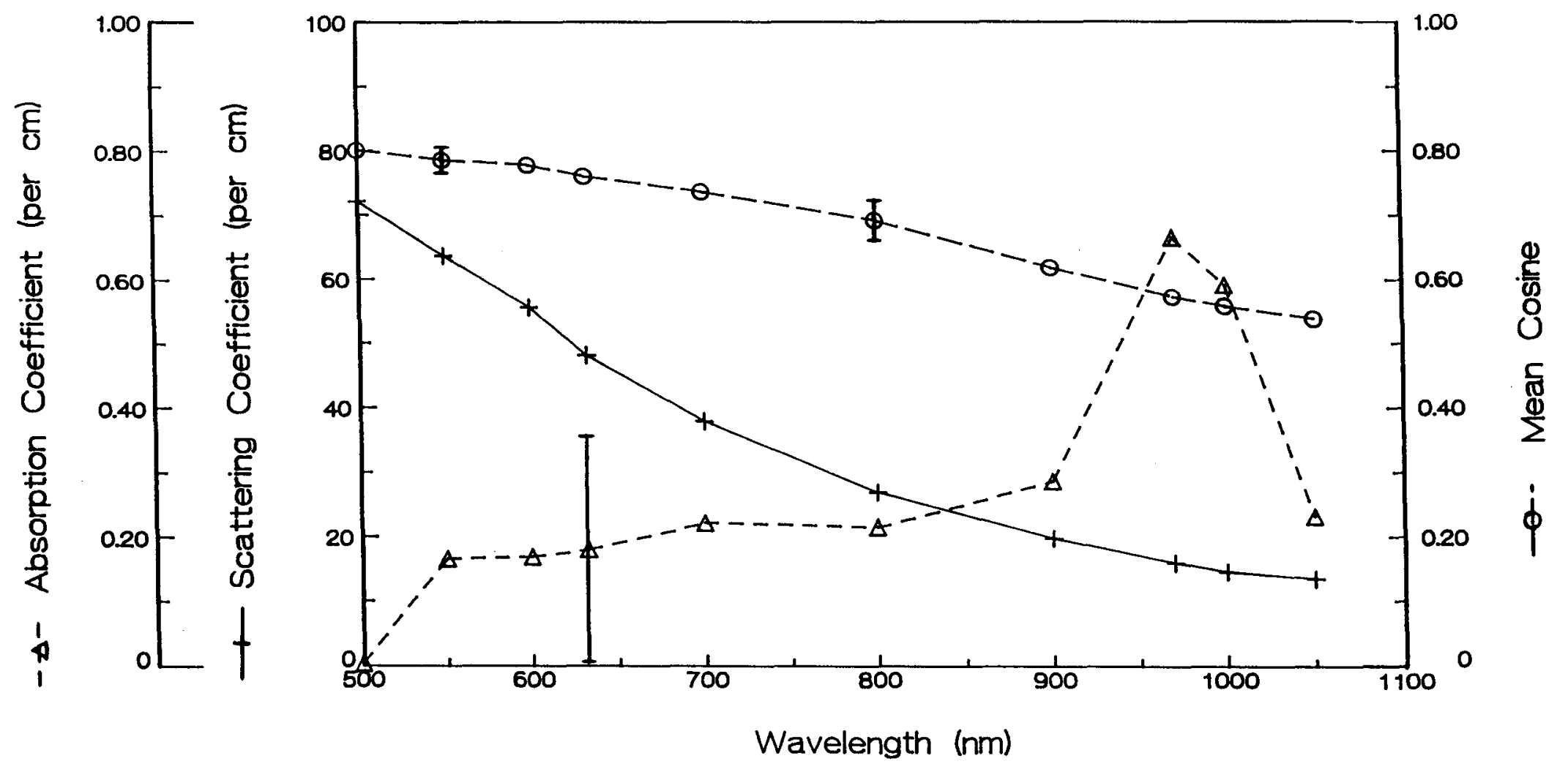

Figure 4.5 Optical properties of Intralipid (1.0 percent solids) derived from the Monte Carlo model. Typical error bars are shown. Errors in the scattering coefficient are smaller than the size of the symbol. 


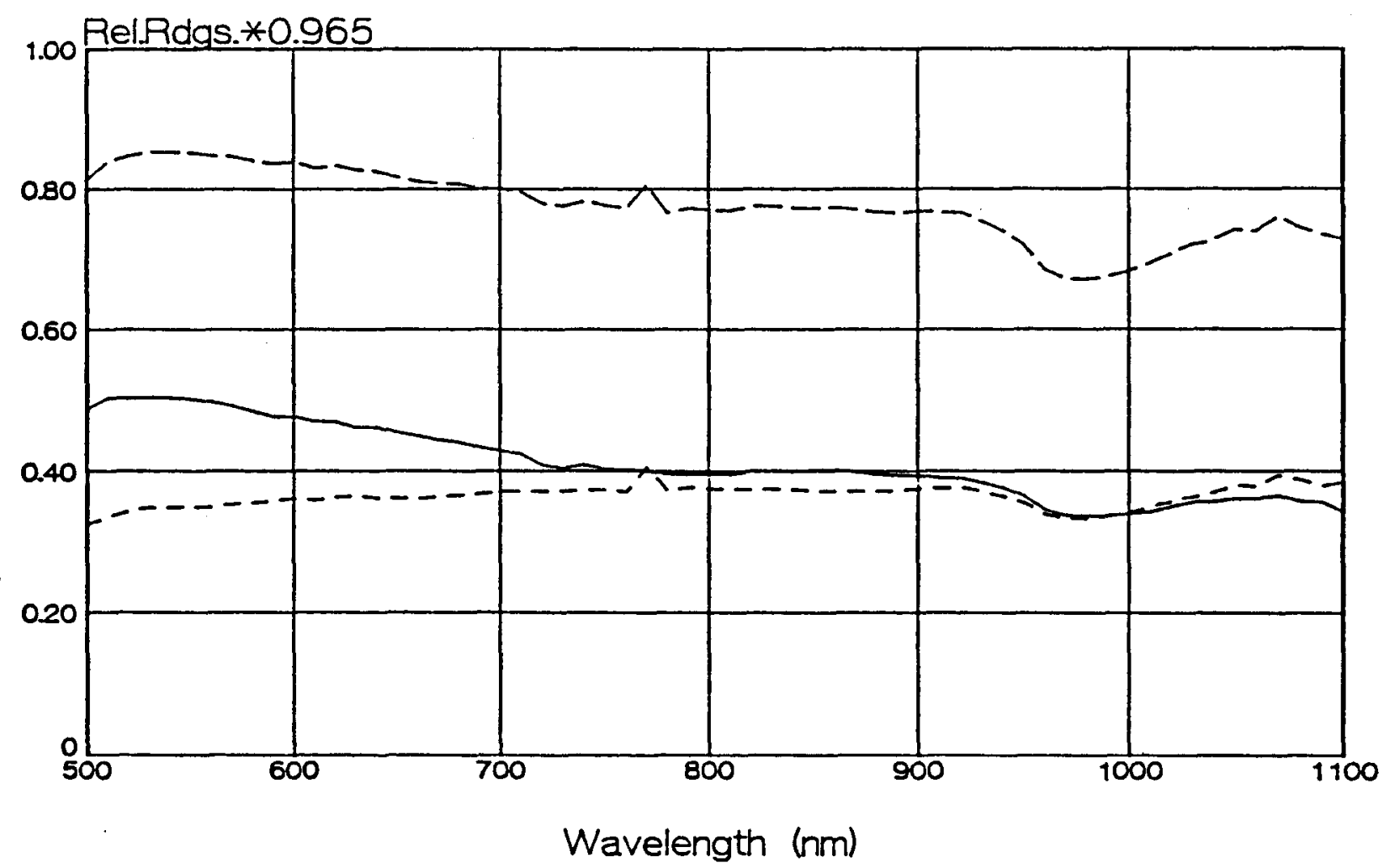

Figure 4.6 Measured reflectance (R) and transmittance (T) of polystyrene microspheres, $1000 \mathrm{~nm}$ in diameter

( $1.0 \mathrm{~mm}$ thick sample containing 0.95 percent spheres by volume). 
As for Intralipid, the attenuation data were well described by straight lines intersecting at the origin. The derived optical properties, including typical error bars, are presented in figure 4.7. Within the experimental errors, the absorption coefficients are identical with those of Intralipid, with an absorption peak centered at $970 \mathrm{~nm}$. The magnitudes of the scattering coefficient and the mean scattering cosine differ from those for Intralipid, although the dependence on wavelength is similar. The optical properties were also calculated using Mie theory. A comparison of the scatter coefficients, obtained by the two methods, is presented in figure 4.8 . These results show excellent agreement.

The derived mean cosine of the scattering angle is compared to the theoretical value in figure 4.9. Each set of points corresponds to a suspension of spheres with a different concentration of added ink. This data is plotted as a function of the $g$ values obtained from Mie theory for the spheres alone. Since ink does not contribute to 1 ight scattering, it is expected that the derived $g$ values will be independent of ink concentration. This result is confirmed for the two suspensions having lower ink concentrations. These suspensions also demonstrate reasonable agreement with theory. For the suspension containing 0.1 percent ink, the derived $g$ values are lower at all wavelengths. 


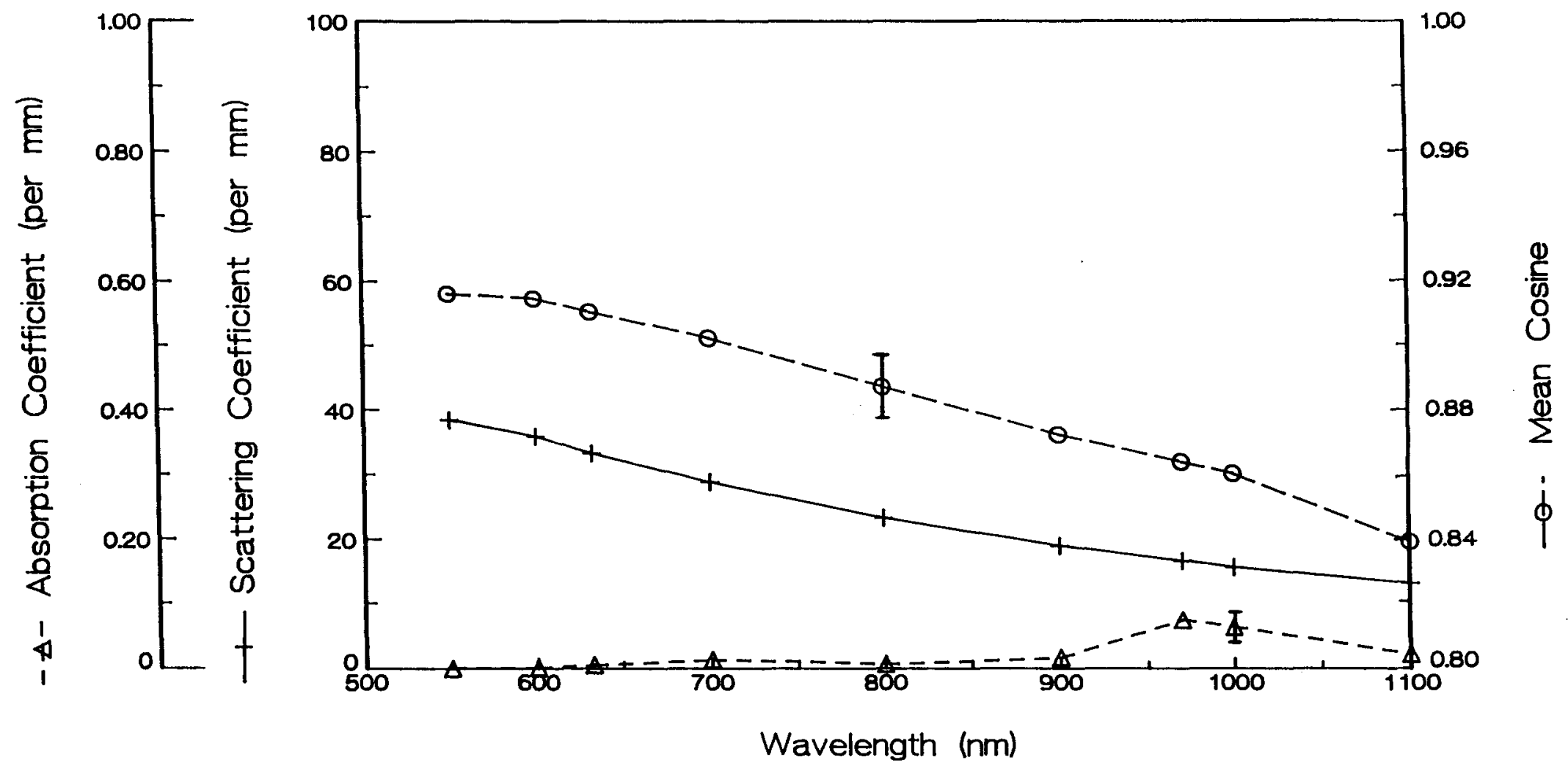

Eigure 4.7 Derived optical properties of polystyrene microspheres, $1000 \mathrm{~nm}$ in diameter $(0.95$ percent spheres by volume).

Typical error bars are shown. For the scattering coefficients

the error bars are smaller than the size of the symbol. 


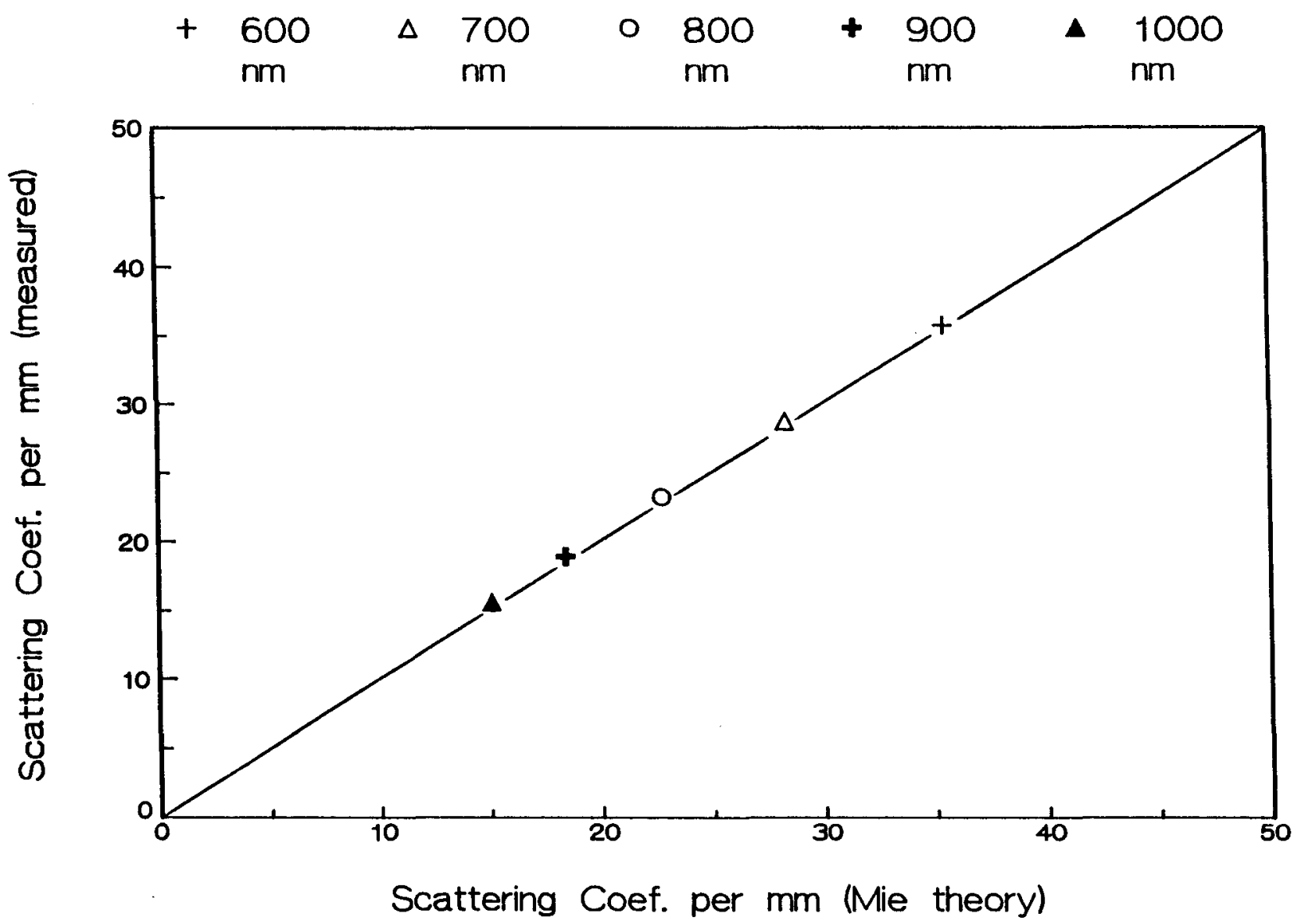

Figure 4.8 A comparison of the measured and theoretical scattering coefficients for polystyrene microspheres, $1000 \mathrm{~nm}$ in diameter ( 0.95 percent spheres by volume). Solid line is the ideal fit. Error bars are smaller than the size of the symbols. 


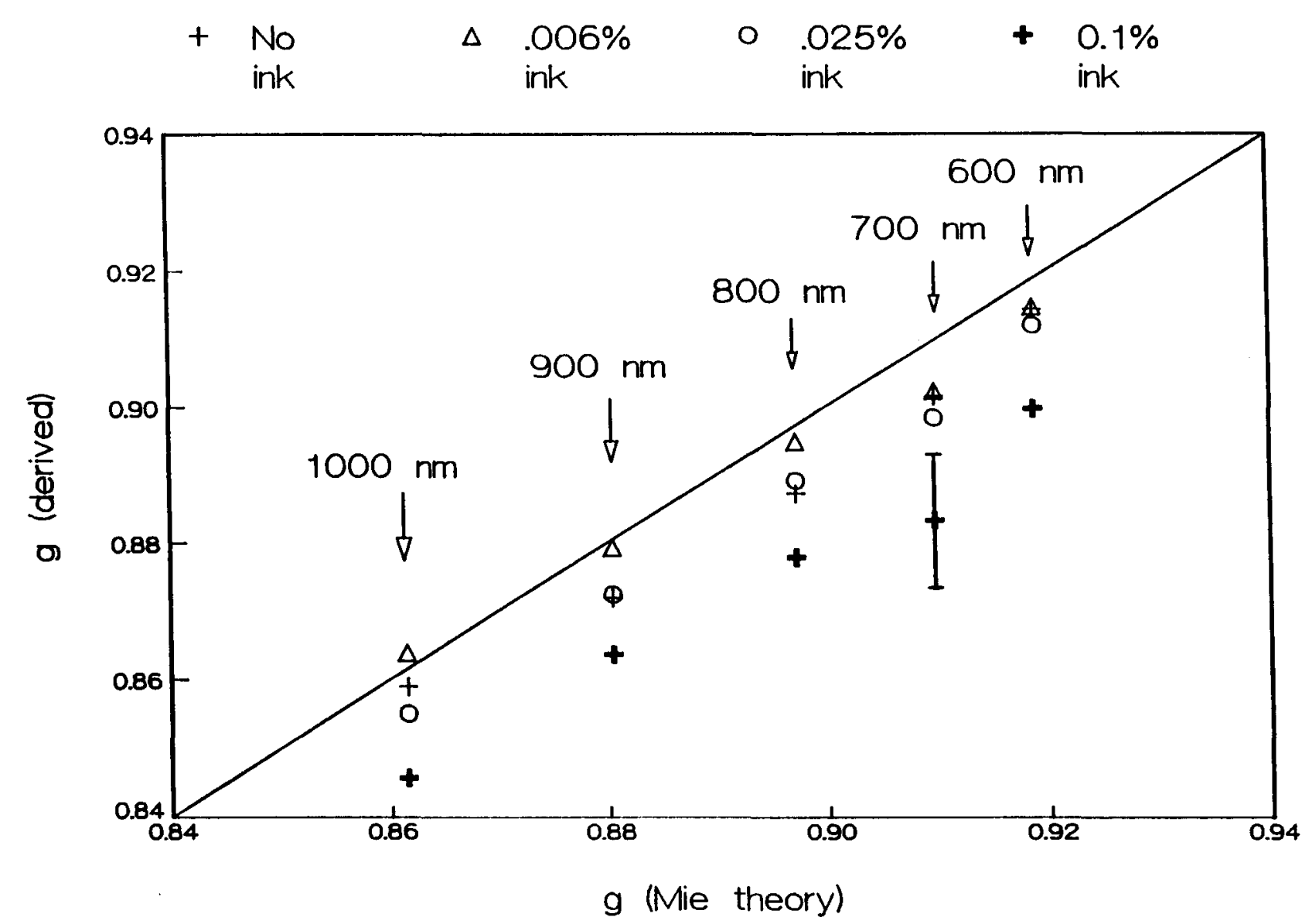

Eigure 4.9 A comparison of the derived and theoretical values for the mean cosine of the scattering angle for a suspension of polystyrene microspheres, with various amounts of ink added as an absorber. The solid line is the ideal fit. The magnitude of the error bar shown is representative of the error for all points. 
The measured and derived absorption coefficients for suspensions containing ink as an added absorber, are compared in figure 4.10. For each ink concentration, the absorption coefficients, as measured in water, are in good agreement with the values derived for ink mixed with polystyrene spheres. Absorption coefficients can be derived with confidence for values up to $1.0 \mathrm{~mm}^{-1}$.

We conclude that our combined experimental and Monte Carlo technique provides good agreement with theory for both scattering and absorption coefficients, as well as for the mean cosine of the scattering angle.

\subsection{Breast tissues}

4.3.1 Effects of tissue preparation

For measurement of reflectance and transmittance, tissue samples were prepared by homogenization as discussed in section 3.4. For two samples (one carcinoma and one fibrocystic disease), these measurements were compared with those made for sliced samples taken from the same specimen. The reflectance and transmittance for the homogenized samples agreed with the corresponding values for the sliced samples within 0.03 . This is within the range of variations measured within any given specimen. It is assumed, therefore, that homogenization did not produce significant changes in the optical properties of the tissues examined. 


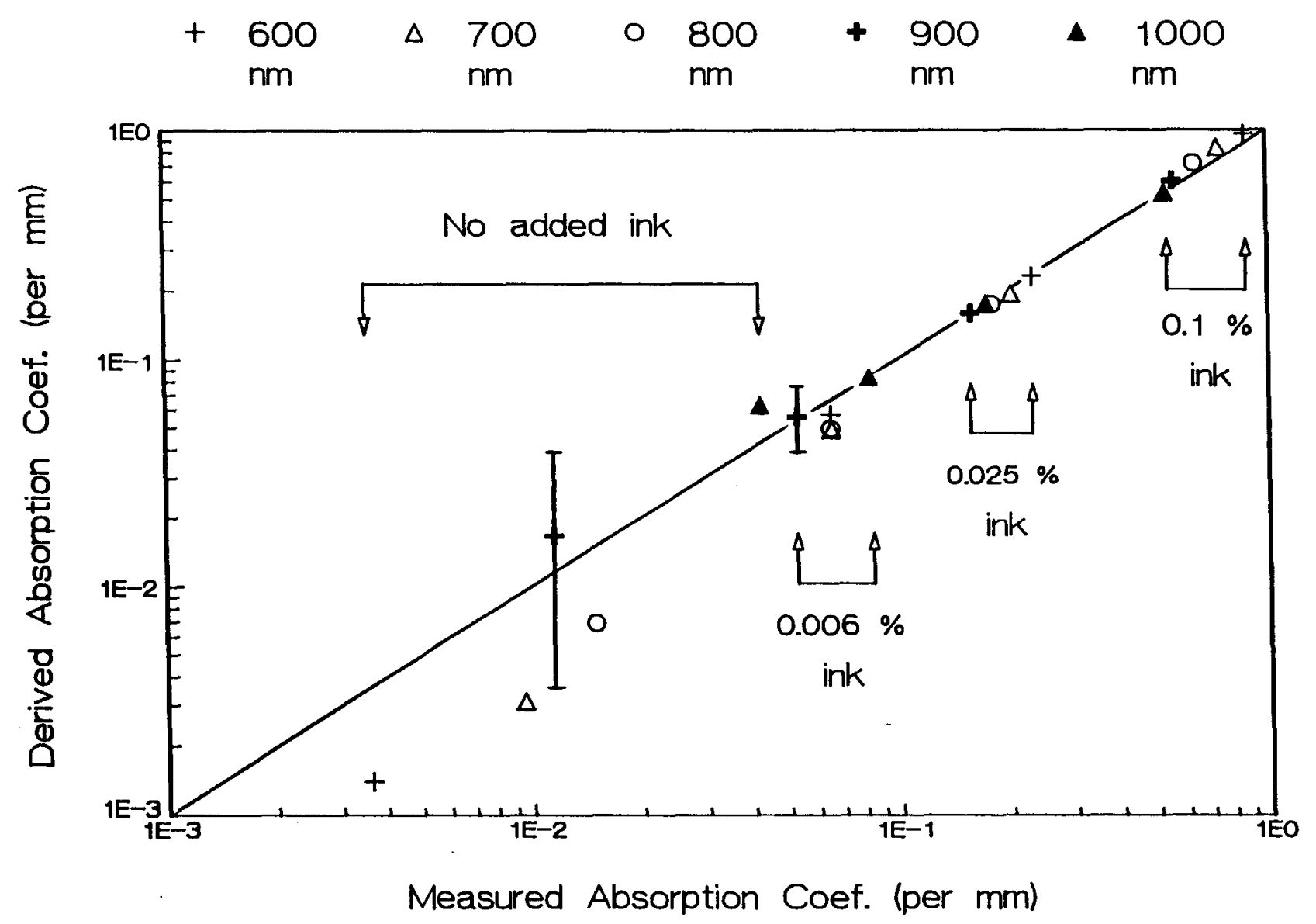

Figure 4.10 A comparison of derived and measured absorption coefficients for suspensions of ink. The solid 1 ine is the ideal fit. Typical error bars are shown. For absorption coefficients greater than $0.1 \mathrm{~mm}^{-1}$, the error bars are smaller than the symbol size. See text for a complete description. 
To determine the effects of freezing and storage, the reflectance and transmittance were measured for freshly prepared samples, which were subsequently frozen. At various times, the samples were thawed and the measurements repeated. For wavelengths greater than $600 \mathrm{~nm}$, the results were repeatable within $+/-0.02$ for periods of time up to six weeks. For wavelengths of $600 \mathrm{~nm}$ or less, the agreement was within $+/-0.05$.

Thin sections, cut by microtome for the determination of attenuation coefficients, were always frozen prior to cutting. On visual inspection, these sections showed varying degrees of homogeneity, and had a tendency to deteriorate more quickly than the thick samples. These samples were always measured immediately following preparation.

4.3.2 Reflectance and transmittance measurements The reflectance and transmittance were measured for a total of 33 specimens. Figure 4.11 shows the results of these measurements for a single specimen from each of the five pathologies investigated.

Each sample in figure 4.11 shows reduced values of both $R_{M}$ and $T_{M}$ at wavelengths below $600 \mathrm{~nm}$, indicating increased absorption of light. For some of the samples, two distinct absorption peaks can be identified at $540 \mathrm{~nm}$ and $570 \mathrm{~nm}$. The position of these peaks corresponds with known 
a)

- R

$---T$

$--\cdot R+T$

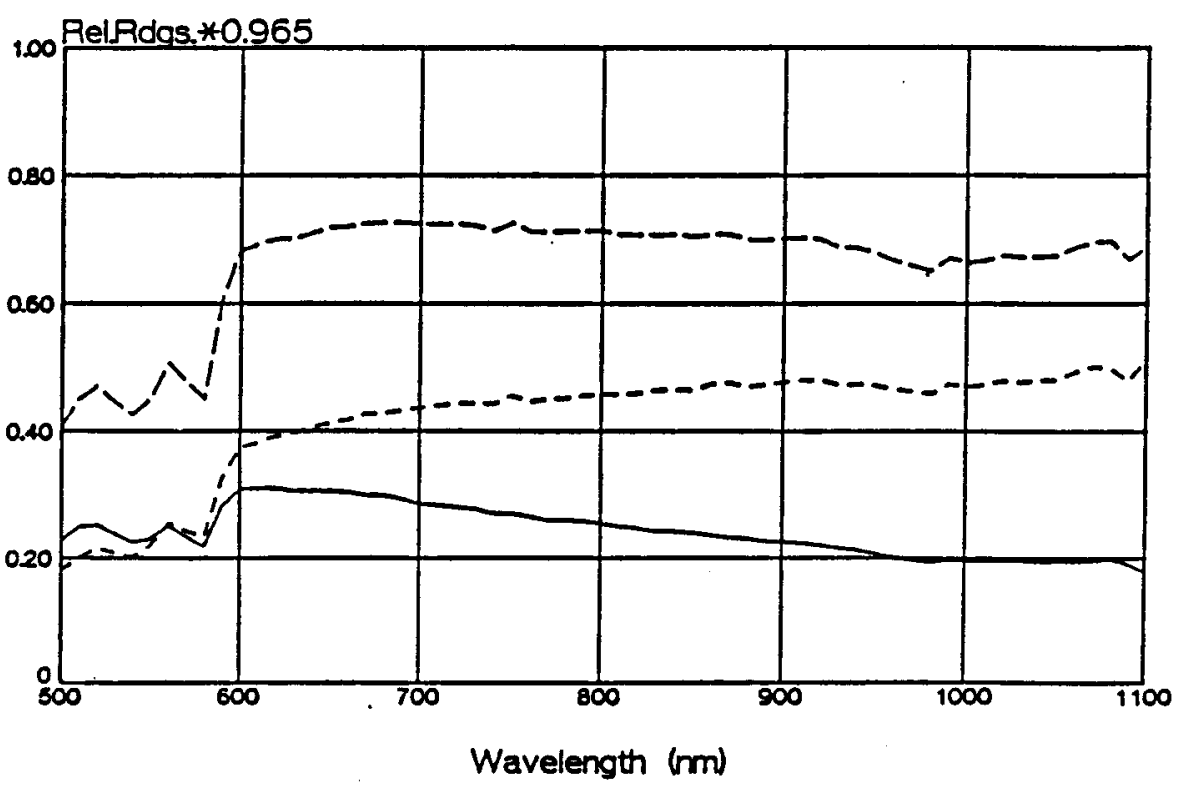

b)<smiles>CP</smiles>

$--\mathrm{T}$

$--R+T$

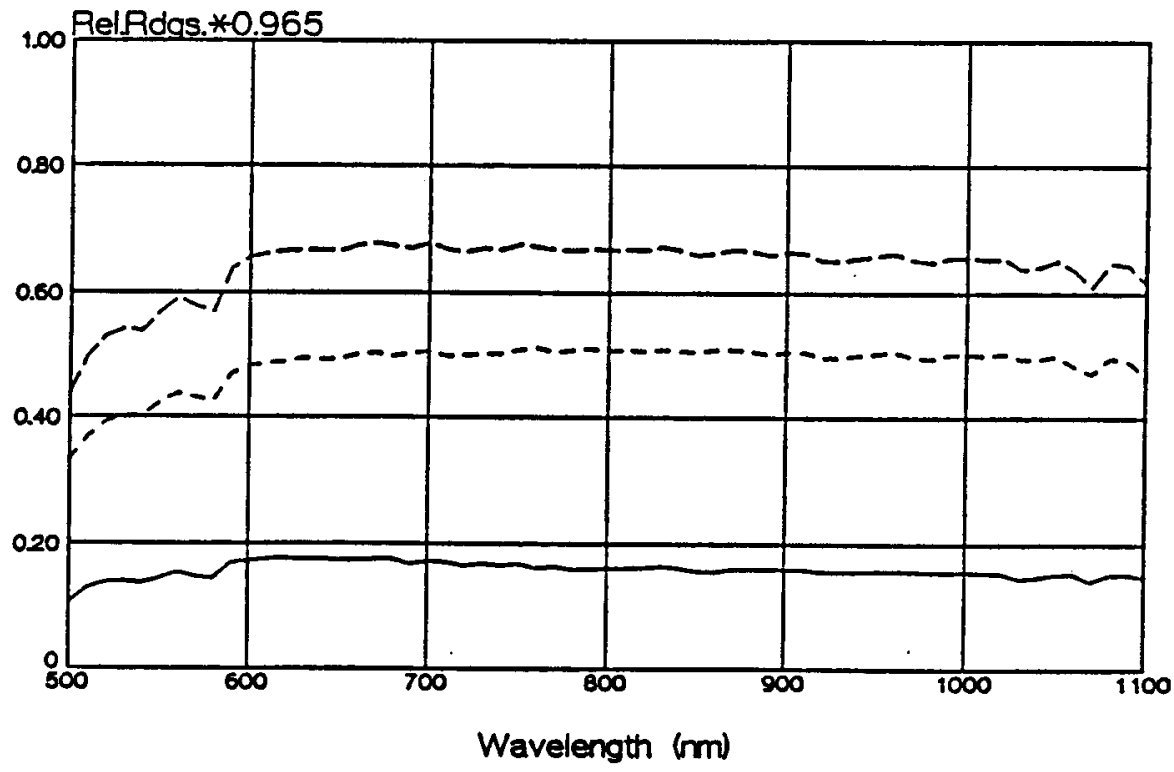

Figure 4.11 Measured reflectance (R) and transmittance (T) for a) normal glandular breast tissue

b) normal breast adipose tissue 
c)

-R

$-\cdots T$

$\sim-A+T$

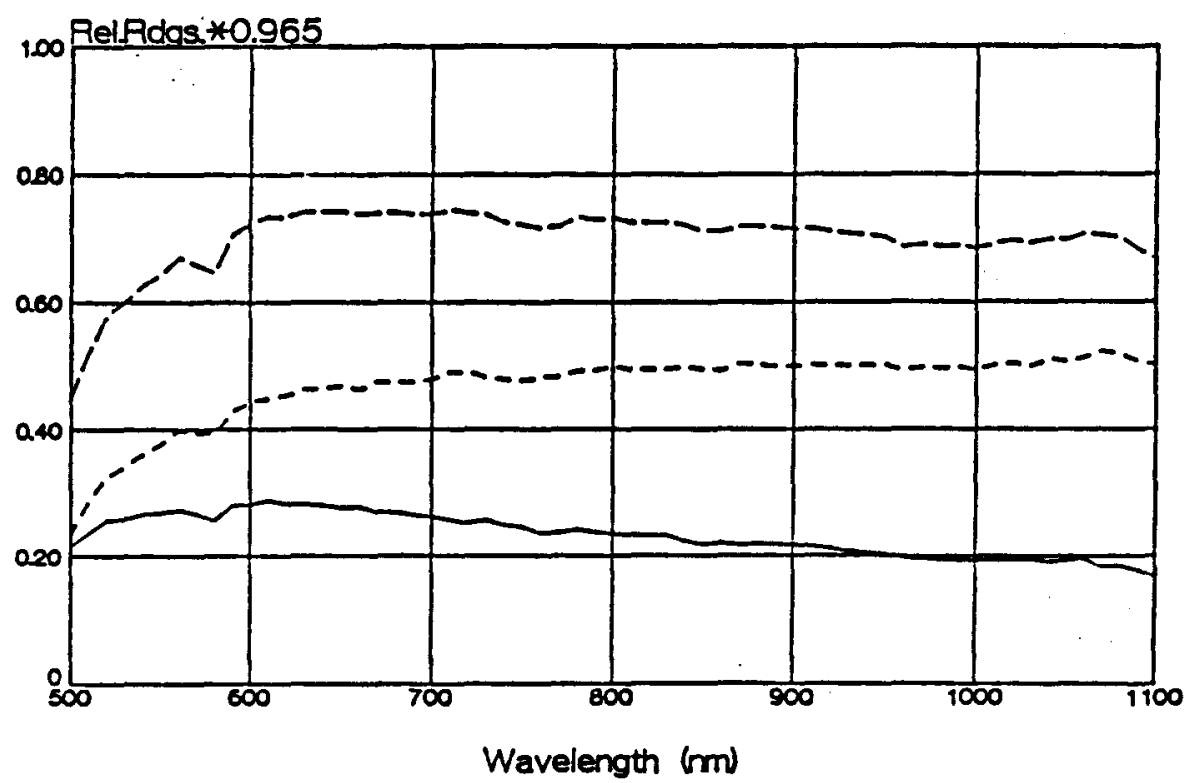

d)

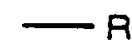

$--T$

$-\cdots R+T$

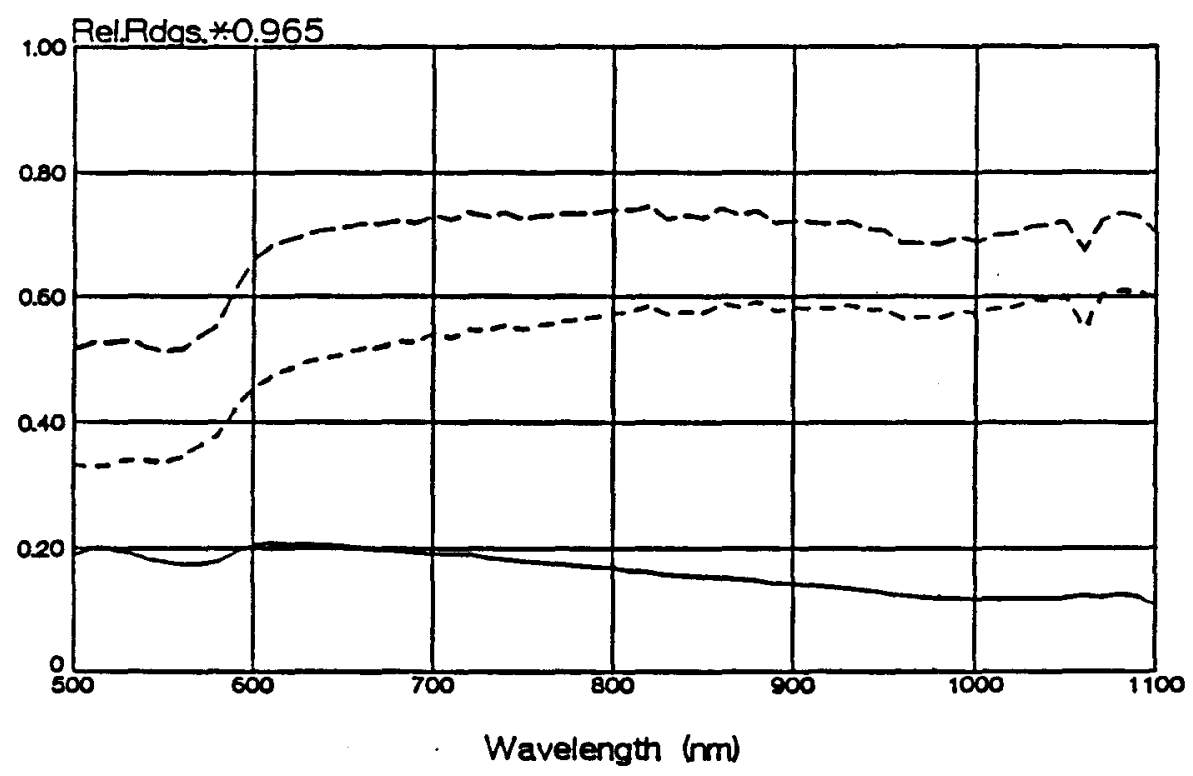

Figure 4.11 (continued)

c) fibrocystic disease

d) fibroadenoma 
e)

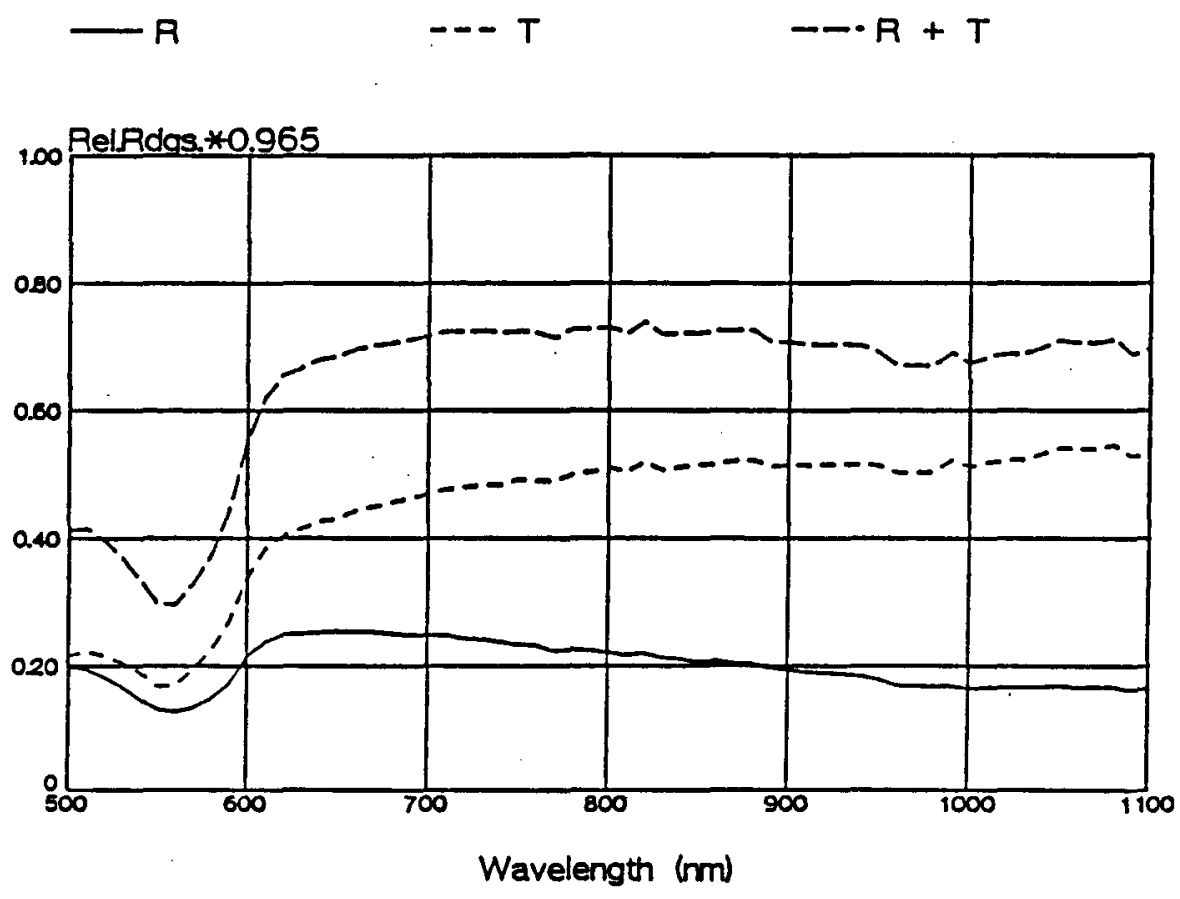

Figure 4.11 (continued)

e) carcinoma 
absorption peaks for oxyhemoglobin. An extreme example of the effect of hemoglobin is illustrated in figure 4.12. $R_{m}$ and $T_{m}$, for a sample of adipose tissue, are compared with similar measurements for tissue from the same specimen mixed with blood. The absorption is affected most significantly at wavelengths below $600 \mathrm{~nm}$, although some increase in absorption is observed across the entire spectrum. Two smaller absorption peaks are visible at about $660 \mathrm{~nm}$ and $760 \mathrm{~nm}$. These peaks correspond with transmission minima reported by Ertefai and Profio (1985) for deoxyhemoglobin.

Significant absorption was observed at wavelengths less than $600 \mathrm{~nm}$ for all 33 samples. A comparison of the behavior of tissues at $540 \mathrm{~nm}$ is provided in figure 4.13, where transmittance has been plotted as a function of the reflectance. The data points for each tissue type are fairly well grouped. Normal adipose tissues, fibrocystic disease, and carcinoma are clearly distinguishable. Normal glandular tissue, represented by only three data points, appears to be similar to carcinoma. Data points for fibroadenoma, although distinct from all tissue types except fat, are not as well clustered. These results suggest a difference in the optical properties of some breast tissues, although the extent to which this differentiation depends upon blood concentration is unclear. 
a)

- Ref.

--- Trans.

$--R+T$

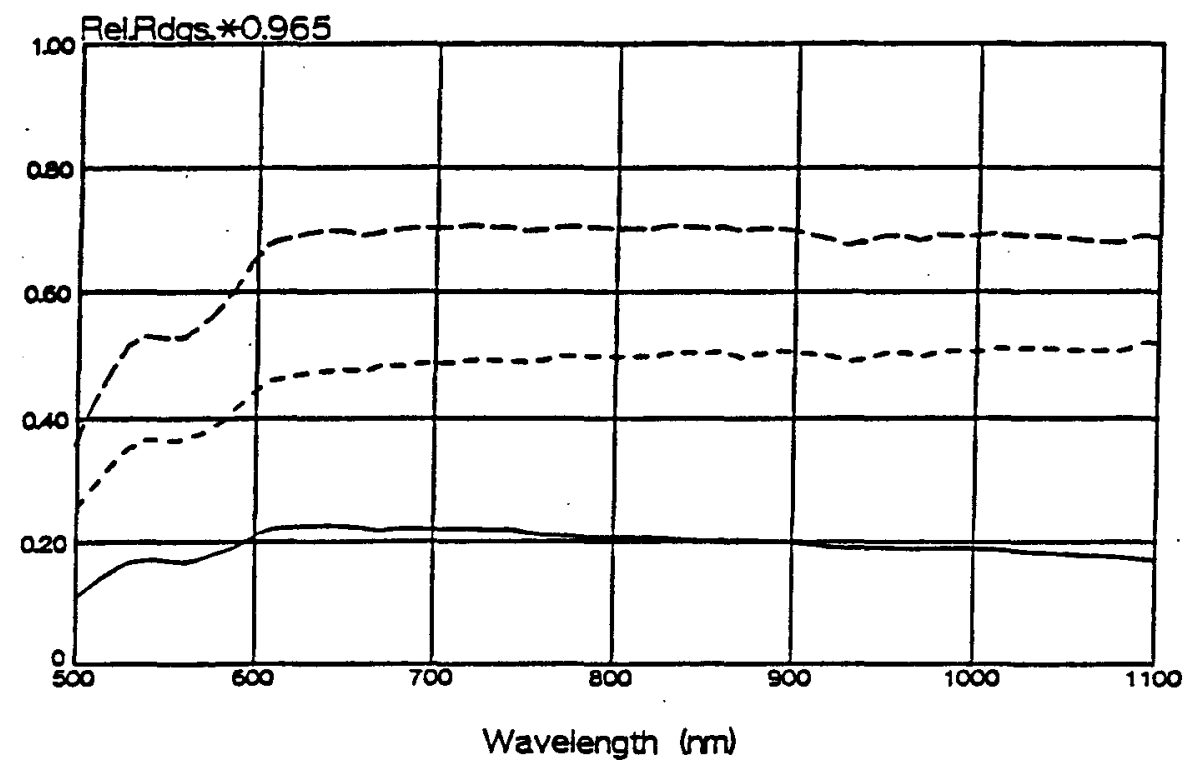

b)<smiles>C[GeH2]</smiles>

--- Trans.

- $R+T$

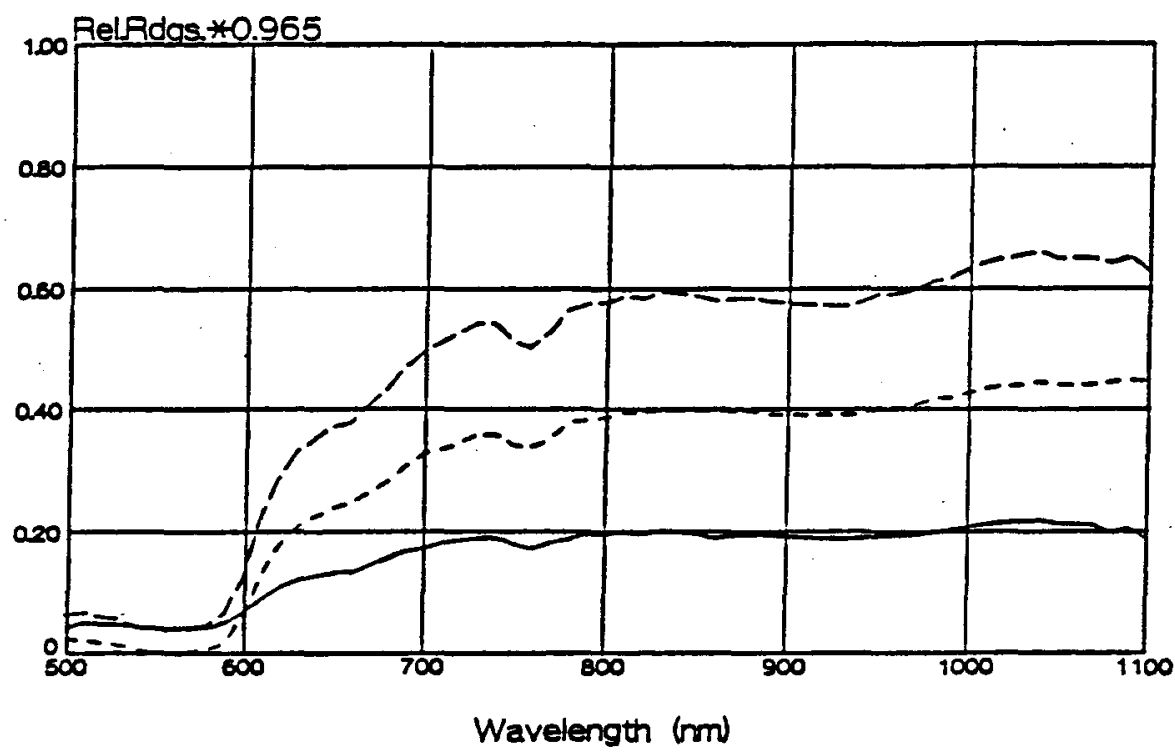

Figure 4.12 a) Measured reflectance (R) and transmittance (T) for normal breast adipose tissue.

b) As in (a) for the same tissue specimen but, in this case, highly pigmented with blood. 


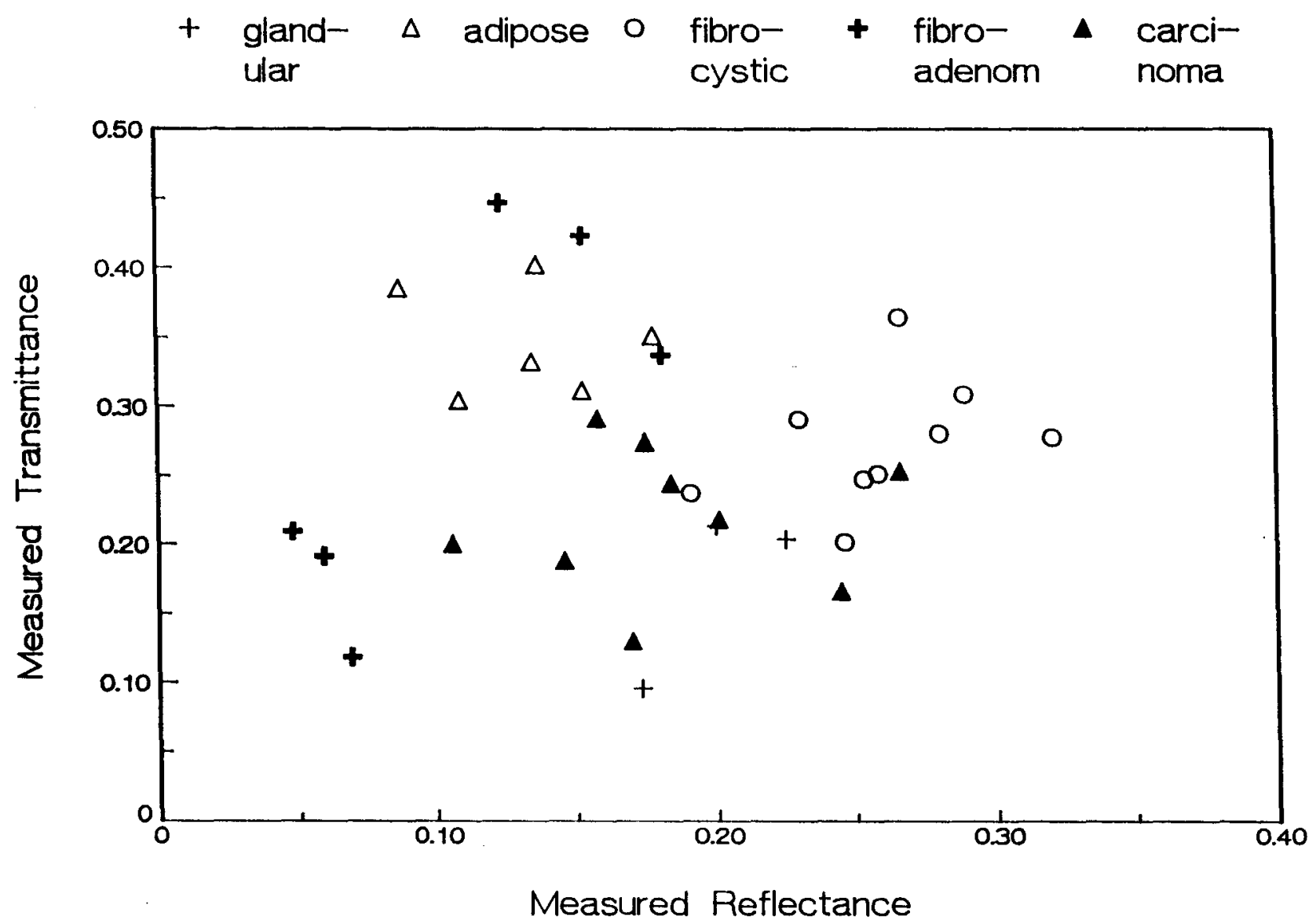

Figure 4.13 Transmittance plotted as a function of reflectance, for 33 samples, at a wavelength of $540 \mathrm{~nm}$. 
The water absorption peak at $970 \mathrm{~nm}$, previously observed for Intralipid, is also present in most of the measured samples. A notable exception is adipose tissue. All adipose samples showed little trace of this peak. This is in agreement with Ertefai and Profio (1985), who also noted less absorption by adipose tissue at this wavelength.

Except for the presence of the water absorption peak, for our samples the reflectance and transmittance can be approximated as linear functions of wavelength, at wavelengths greater than $700 \mathrm{~nm}$ (figure 4.11). For the comparison of sample behavior over this region, the transmittance has been plotted as a function of the reflectance in figure 4.14. A separate plot is provided for each pathology. Two data points are plotted for each specimen, representing the results at 700 and $900 \mathrm{~nm}$. For each pathology the reflectance decreases while the transmittance increases with increasing wavelength. This trend was also observed for Intralipid and polystyrene spheres. Adipose tissue displays the smallest change. $R_{M}$ and $T_{m}$ are not identical for all samples of a given pathology, although they do fall into identifiable ranges. At these wavelengths, there appear to be no significant differences in the measured quantities for normal glandular tissues, fibrocystic disease, and carcinoma. In contrast with these tissues, the reflectance values for adipose tissue are lower for any given transmittance. 
a)

$$
\begin{array}{lll}
+ \text { at } & 0 & \text { at } \\
700 \mathrm{~mm} & 900 \mathrm{~mm}
\end{array}
$$

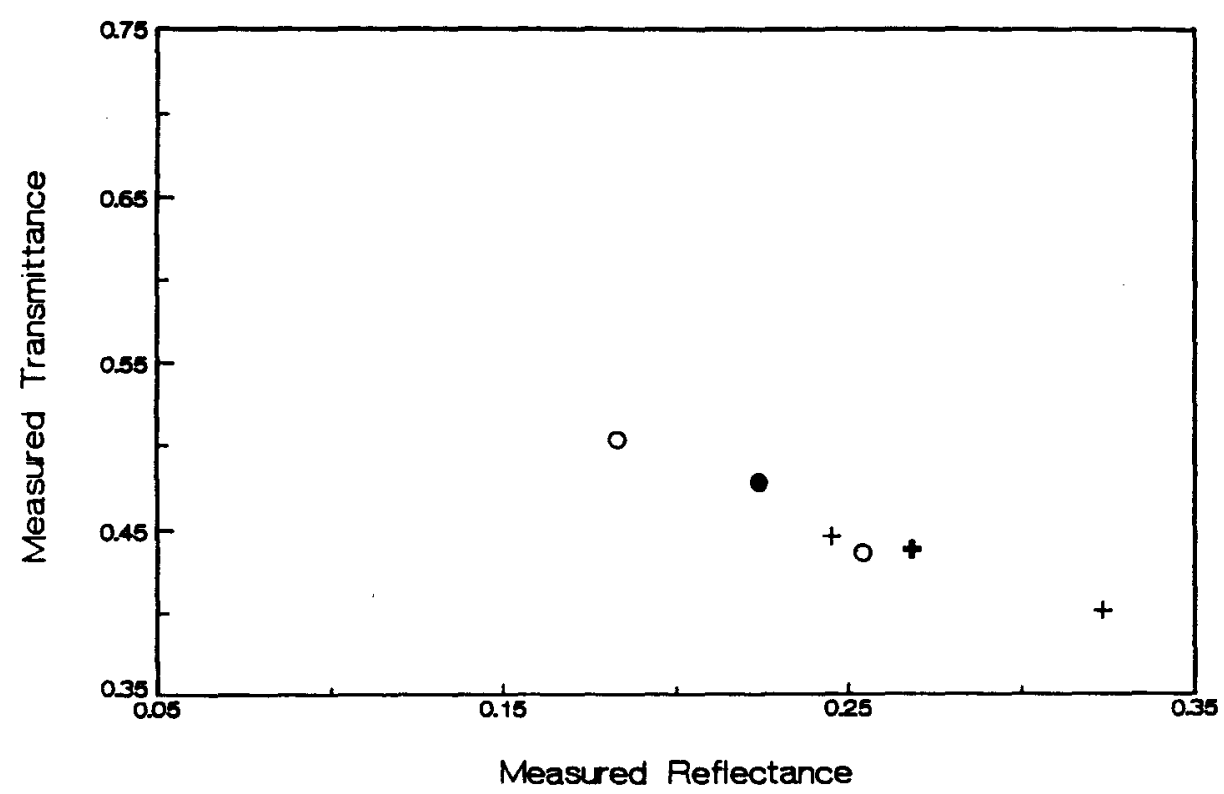

b)

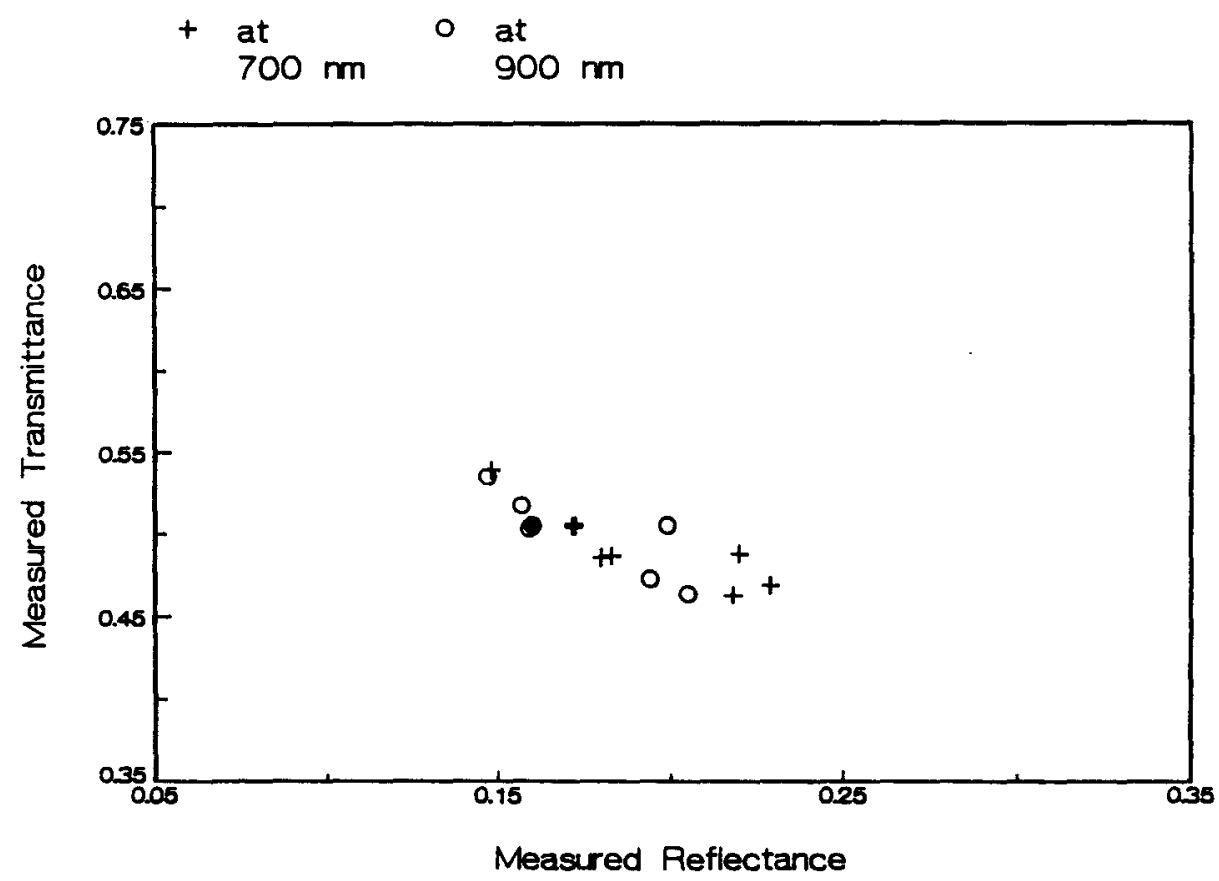

Figure 4.14 Transmittance plotted as a function of reflectance at $700 \mathrm{~nm}$ and $900 \mathrm{~nm}$. Highlighted data points represent the samples presented in figure 4.11. a) normal glandular breast tissue

b) normal breast adipose tissue 
c)

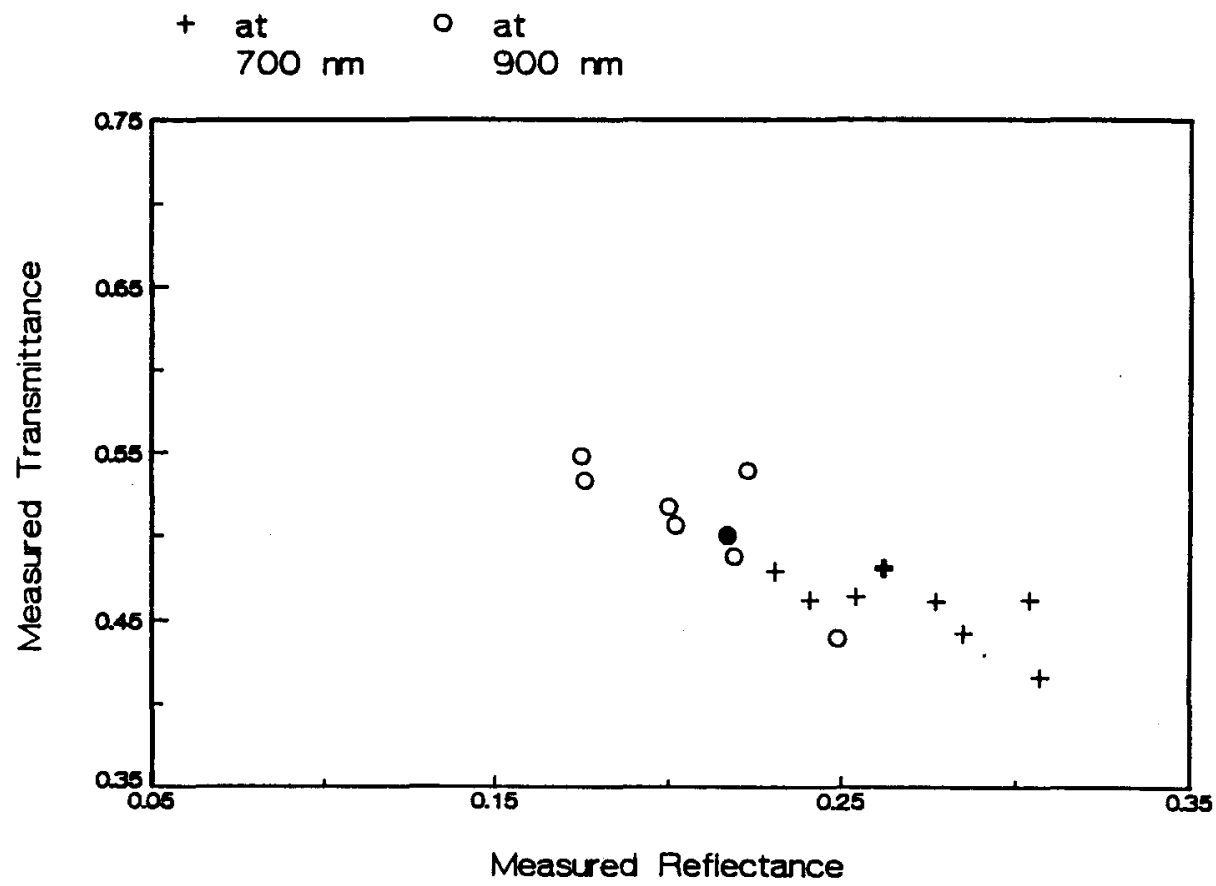

d)

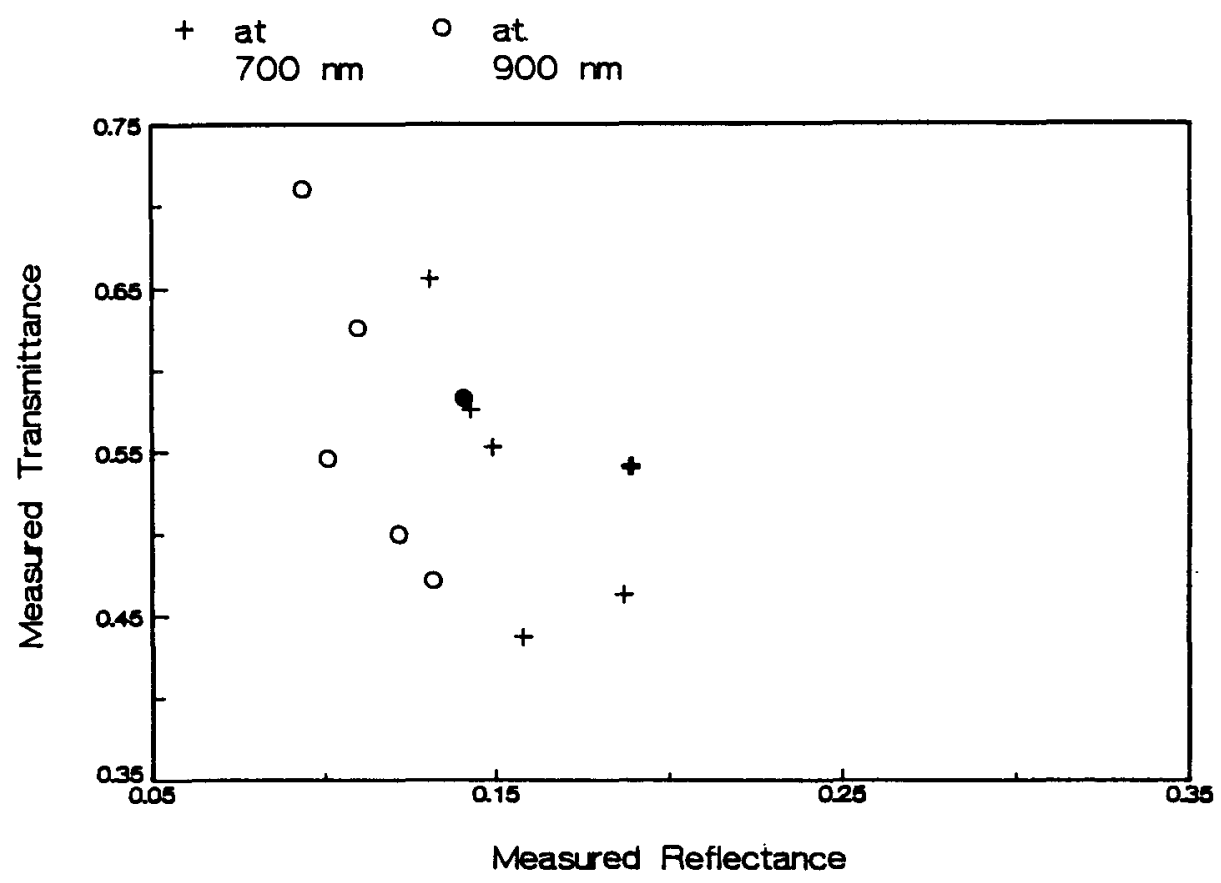

Figure 4.14 (continued)

c) fibrocystic disease

d) fibroadenoma 
e)

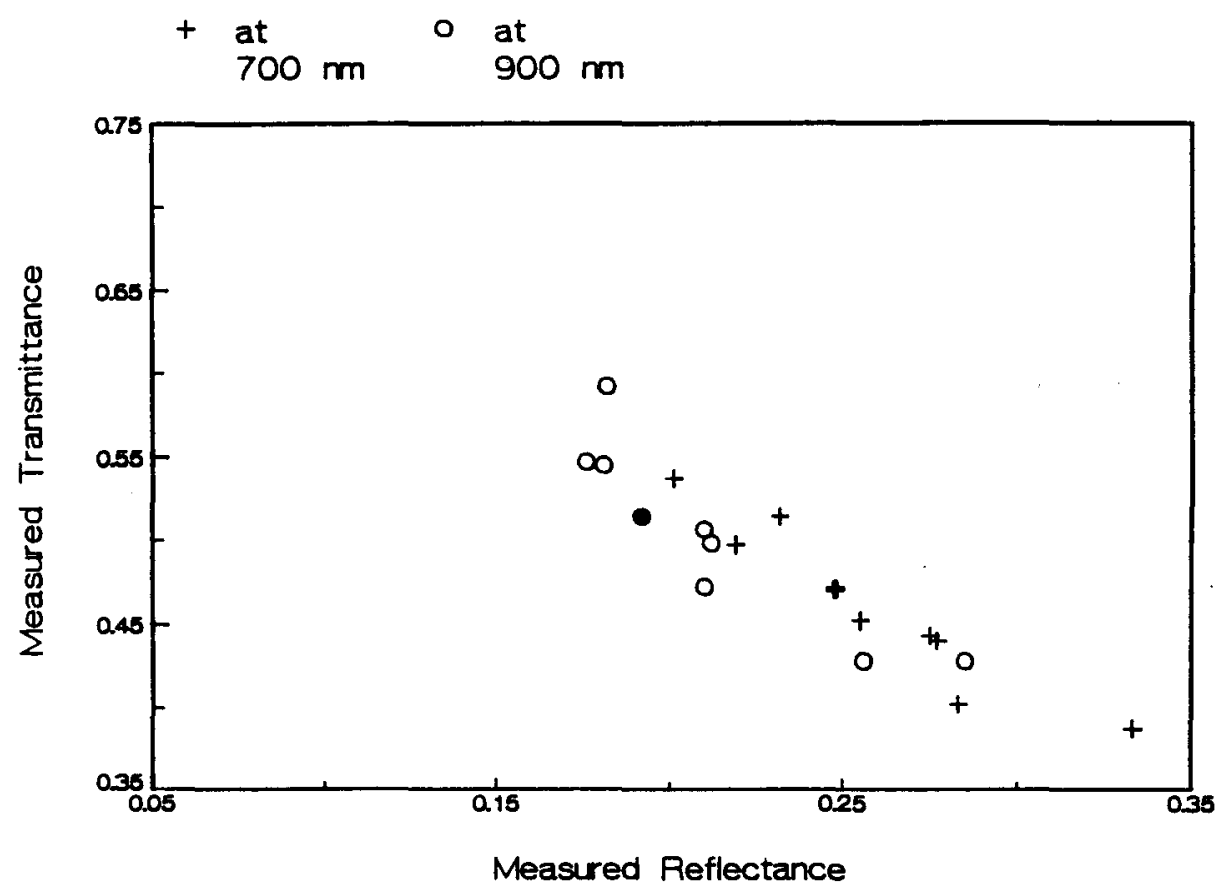

Figure 4.14 (continued)

e) carcinoma 
Fibroadenoma samples display a significant shift in the positions of data points with respect to all other tissues. Variations in the concentration of blood are expected to have less influence on tissue characteristics at 700 and $900 \mathrm{~nm}$ than at $540 \mathrm{~nm}$. Since adipose tissues and fibroadenomas exhibit significant differences from other breast tissues at all three wavelengths, it is unlikely that these results can be attributed solely to the concentration of blood. Fibrocystic disease, while distinct from carcinoma and normal glandular tissues at $540 \mathrm{~nm}$, shows no differences at 700 or $900 \mathrm{~nm}$. It is possible, therefore, that these tissues may differ only in their blood content. The fundamental optical properties are discussed in greater detail in the following sections.

\subsubsection{Total attenuation measurements}

The total attenuation coefficients were determined for each of the five specimens previously discussed (figure 4.11). Attenuation data were collected at $100 \mathrm{~nm}$ increments and are presented for wavelengths of $700 \mathrm{~nm}$ and $900 \mathrm{~nm}$ in figure 4.15. Due to the inhomogeneity of the cut slices, the data points show much more scatter than that observed for Intralipid or the polystyrene spheres. 
a)

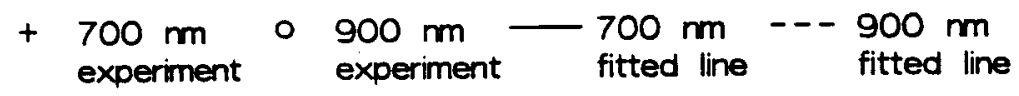

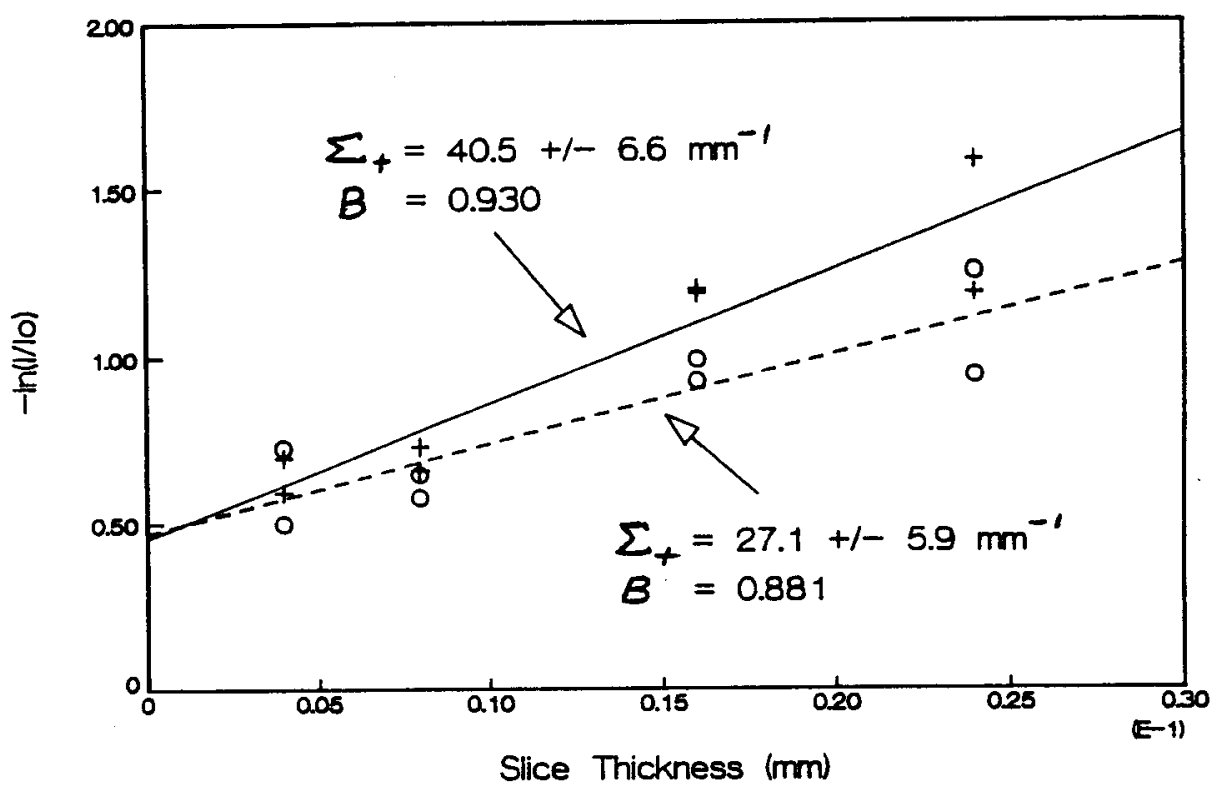

b)

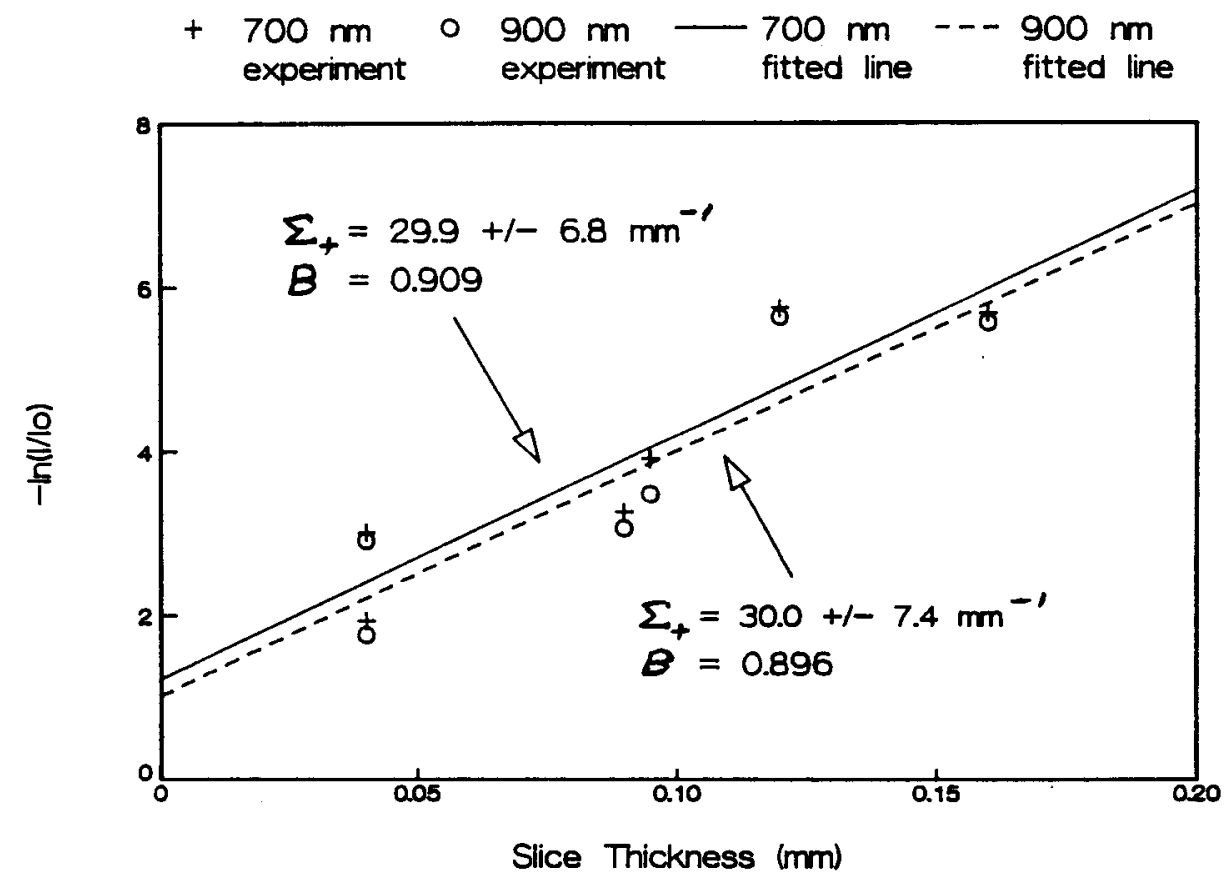

Figure 4.15 Attenuation data at $700 \mathrm{~nm}$ and $900 \mathrm{~nm}$ for five tissue specimens, plotted as a function of slice thickness. The slope of the fitted lines gives the total attenuation coefficient, $\Sigma_{+}$, with regression coefficient $B$.

a) normal glandular breast tissue

b) normal breast adipose tissue 
c)

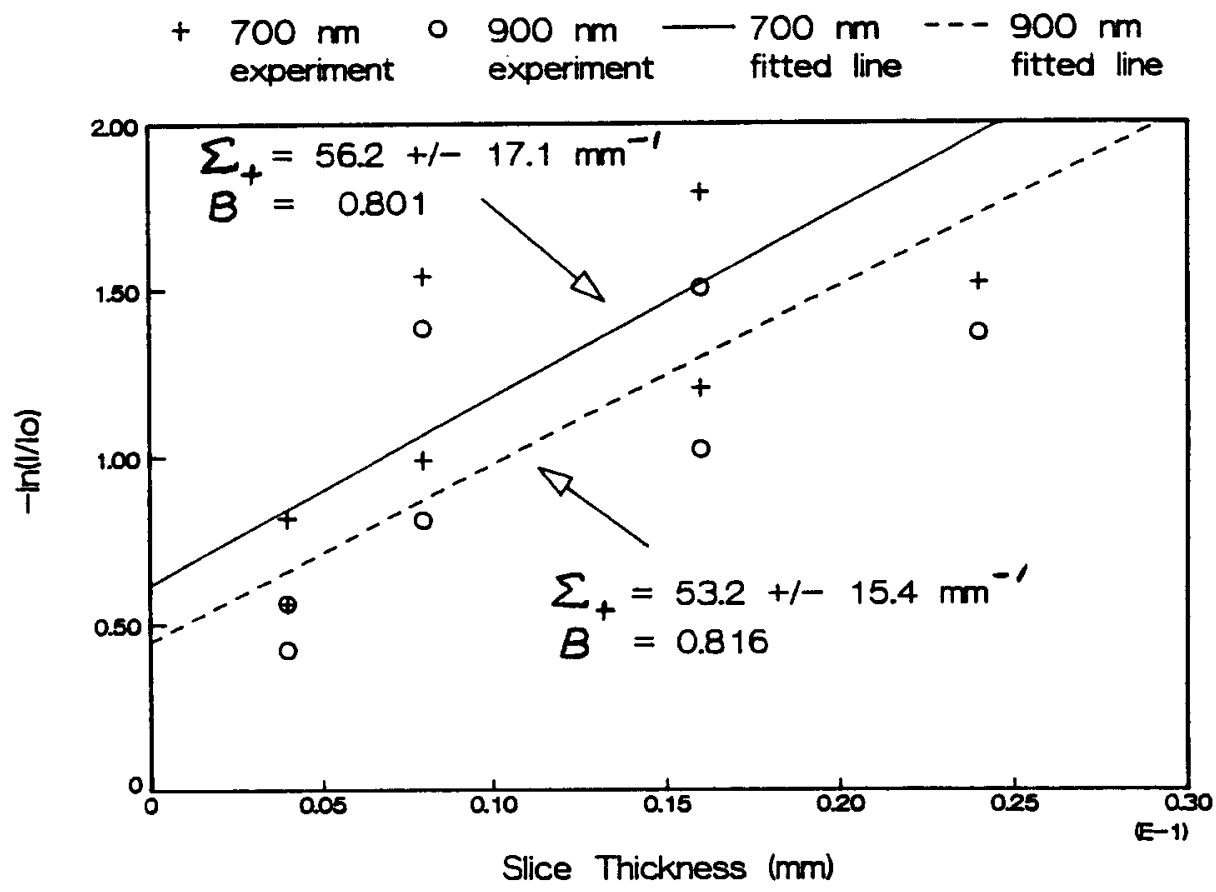

d)

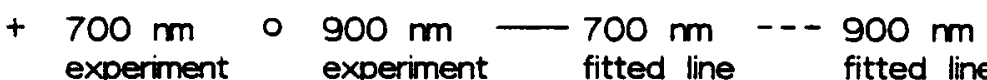

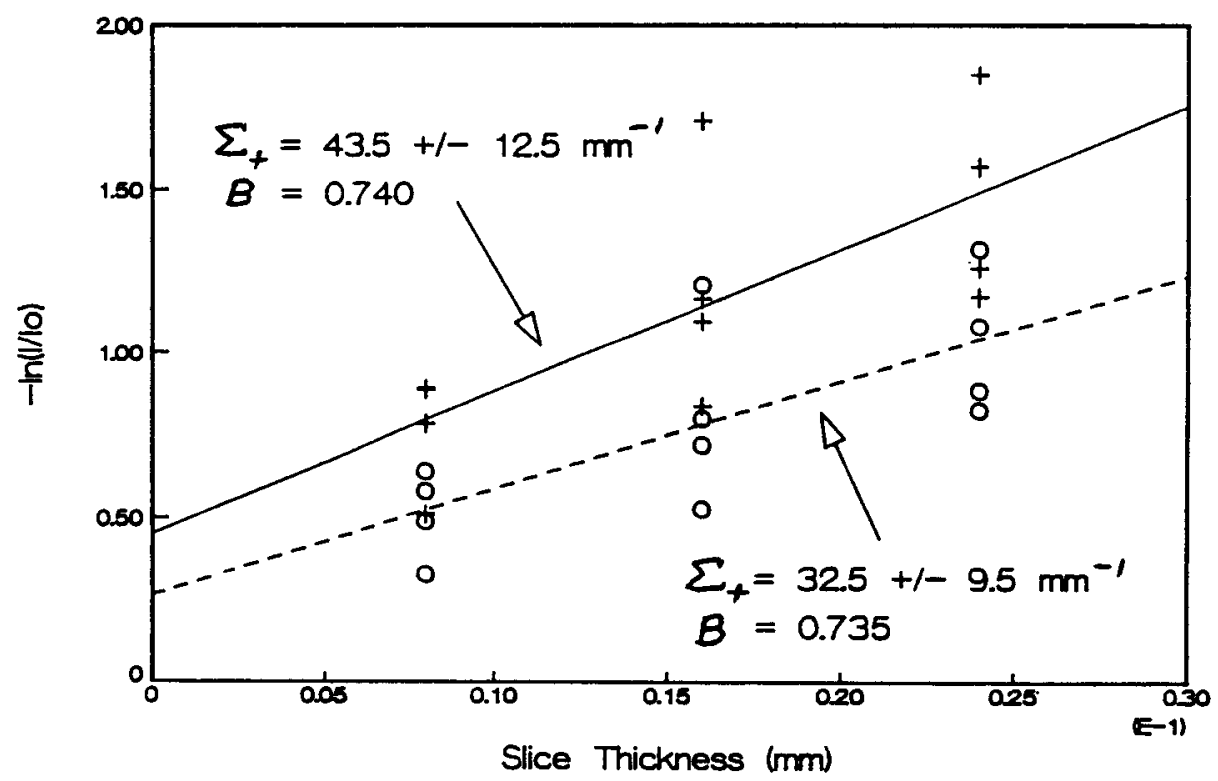

Figure 4.15 (continued)

c) fibrocystic disease

d) fibroadenoma 
e)

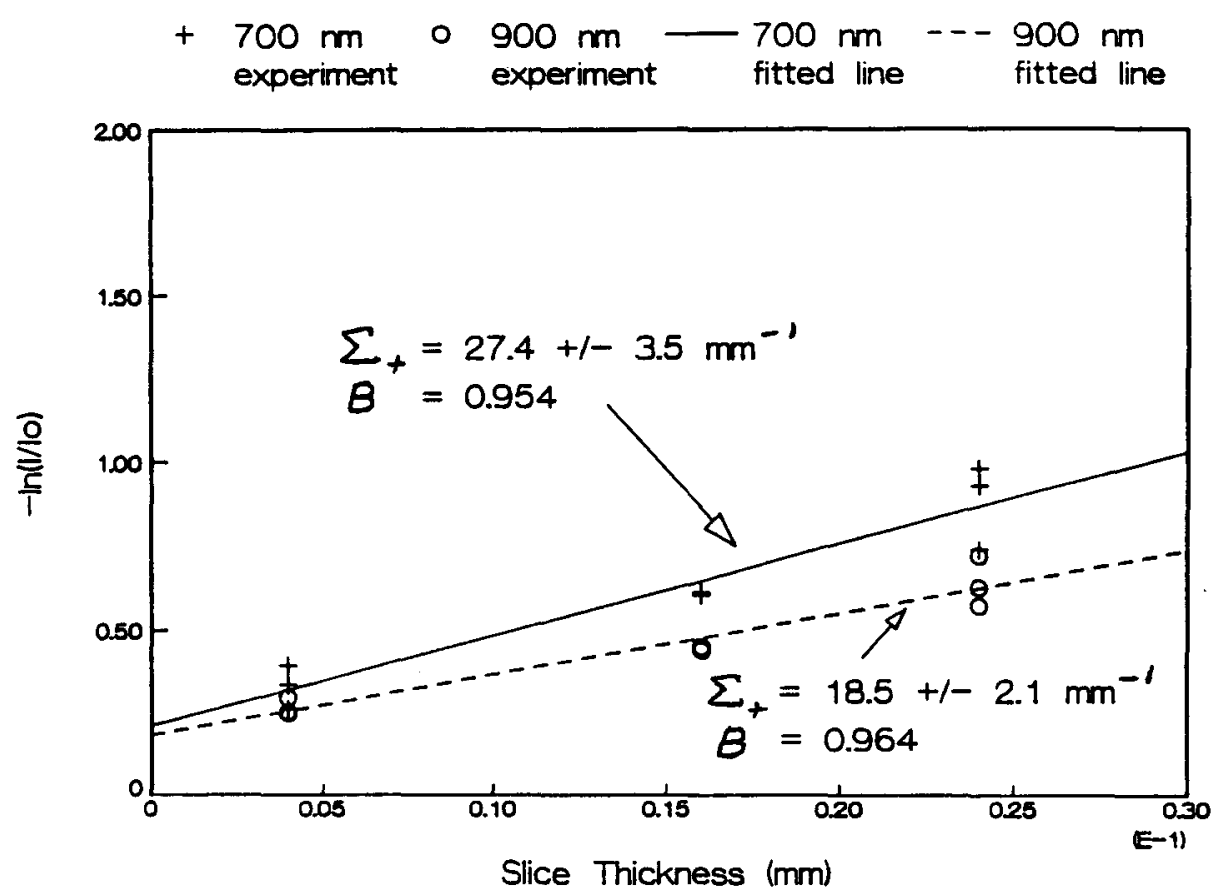

Figure 4.15 (continued)

e) carcinoma 
The points have been fit with least squares regression lines. Extrapolation of these lines to zero sample thickness indicates some attenuation which cannot be attributed to the sample. These losses account for 20 to 70 percent of the incident light, and may be caused by poor coupling of the sample with the glass slides. Variations in coupling efficiency may, therefore, be the cause of some of the scatter in the data.

The attenuation coefficients were obtained from the slope of the fitted 1 ines. At $700 \mathrm{~nm}$ the attenuation coefficient for normal glandular tissue was measured as $40.5 \mathrm{~mm}^{-1}$. This is in excellent agreement with the $25 \mu \mathrm{m}$ mean free path reported by Crilly (1986). At $635 \mathrm{~nm}$, Marchesini et al (1989) reported an attenuation coefficient of $39.5 \mathrm{~mm}^{-1}$ for glandular breast tissue. Our value of $41.8 \mathrm{~mm}^{-1}$ compares very favourably with this result. Similar values were reported by Flock et al (1987), at 633 nm, for bovine muscle and white muscle from chicken.

\subsubsection{Derived optical properties}

The optical properties for the five tissue specimens which have been examined in detail were derived using the Monte Carlo model. The results are presented in figure 4.16. Typical error bars based on the errors in the measured quantities are shown. Errors in the reflectance and transmittance were taken as $+/-0.03$. 
a)

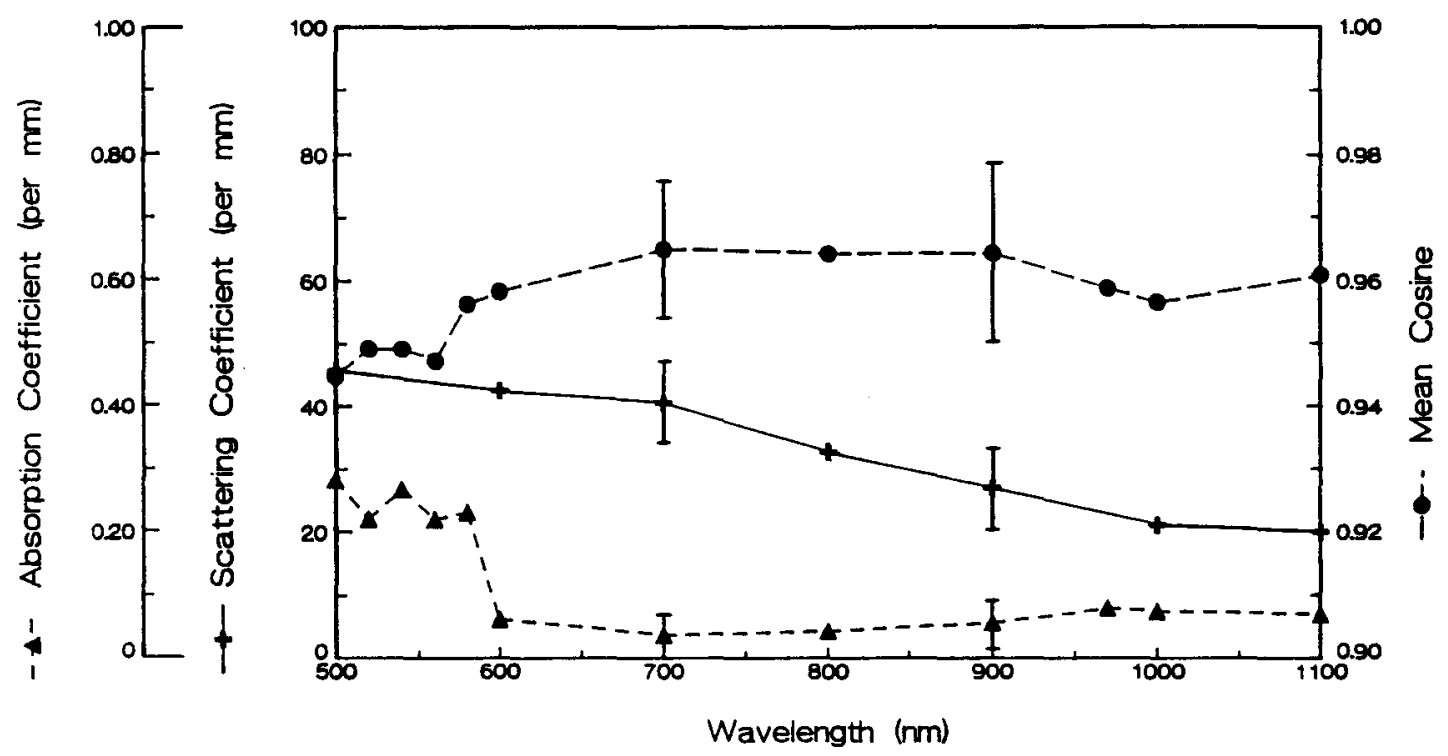

b)

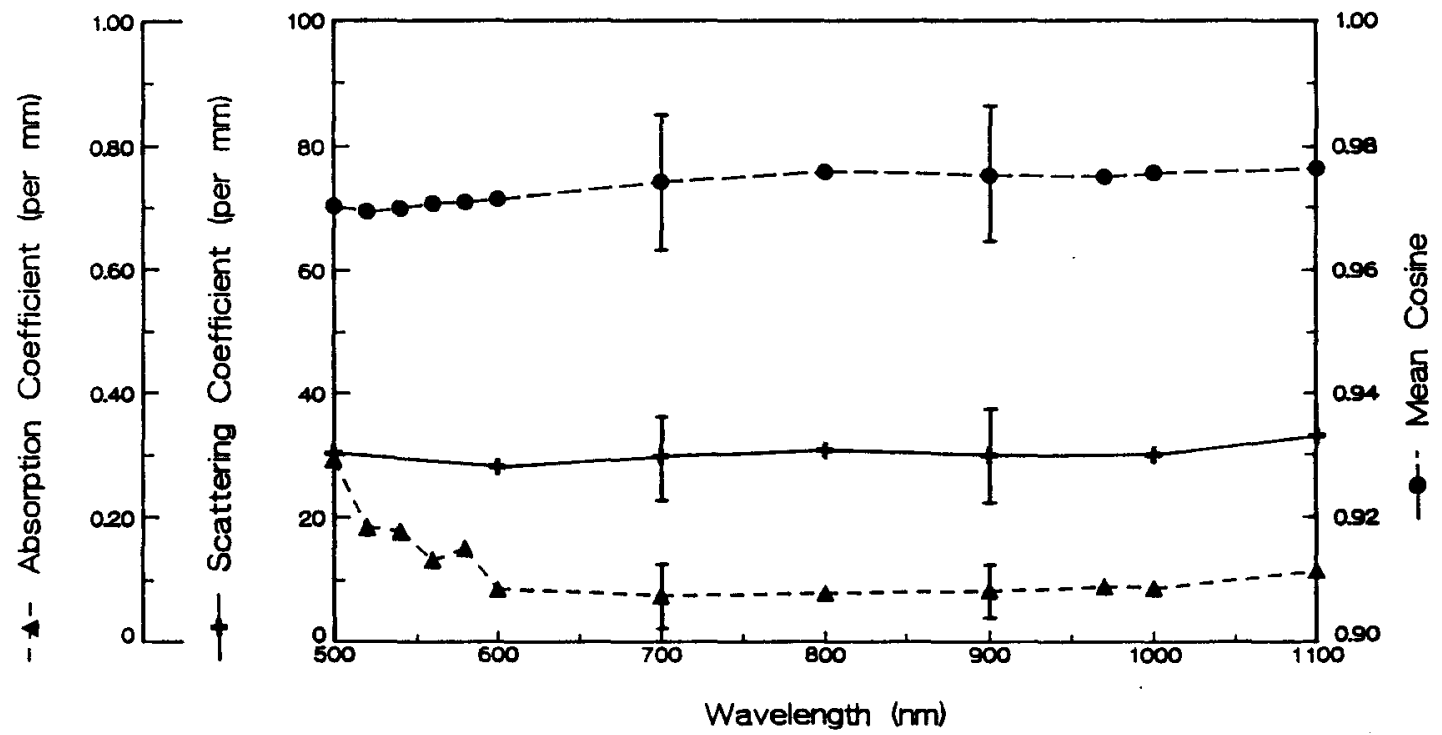

Eigure 4.16 Derived optical properties for five tissue specimens. Each data point is the result of a Monte Carlo calculation. Typical error bars are shown. a) normal glandular breast tissue

b) normal breast adipose tissue 
c)

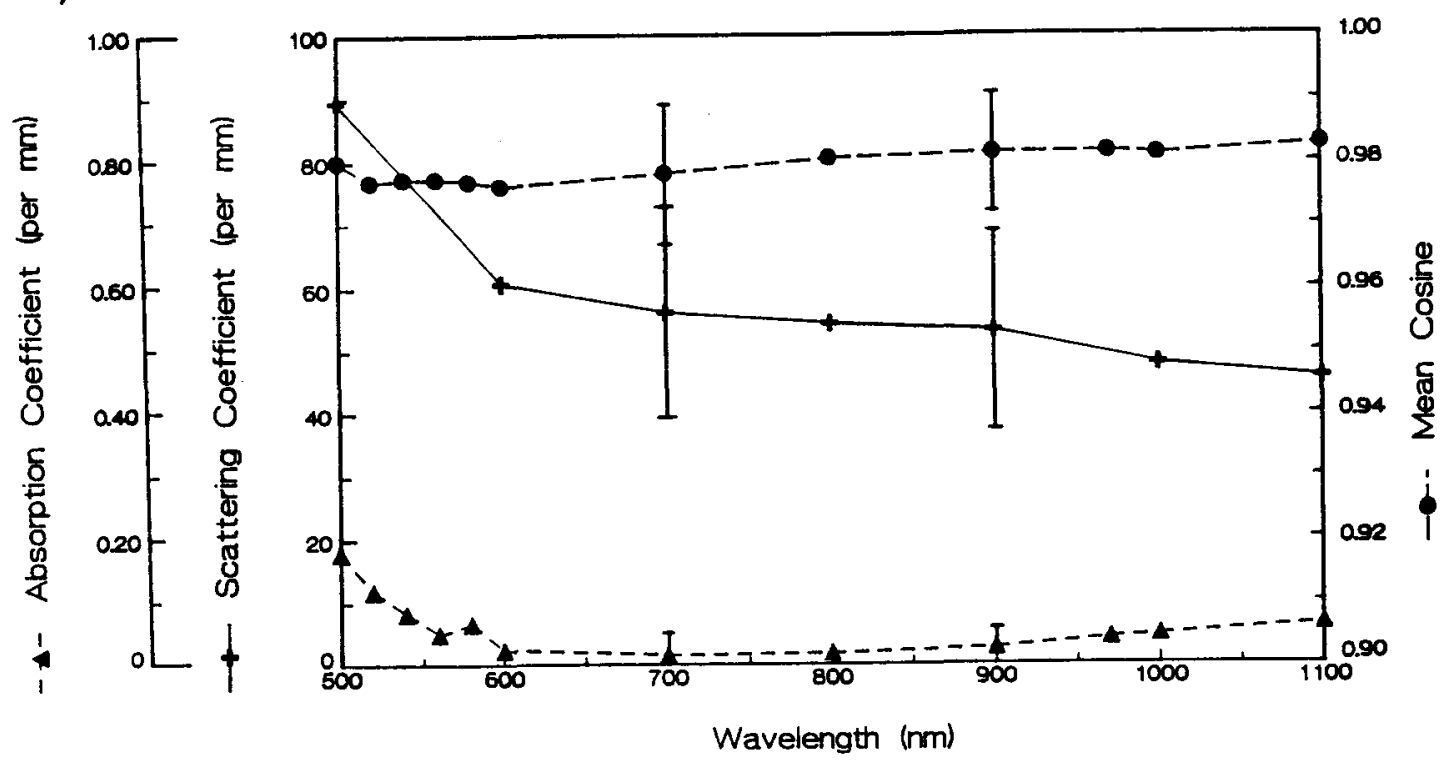

d)

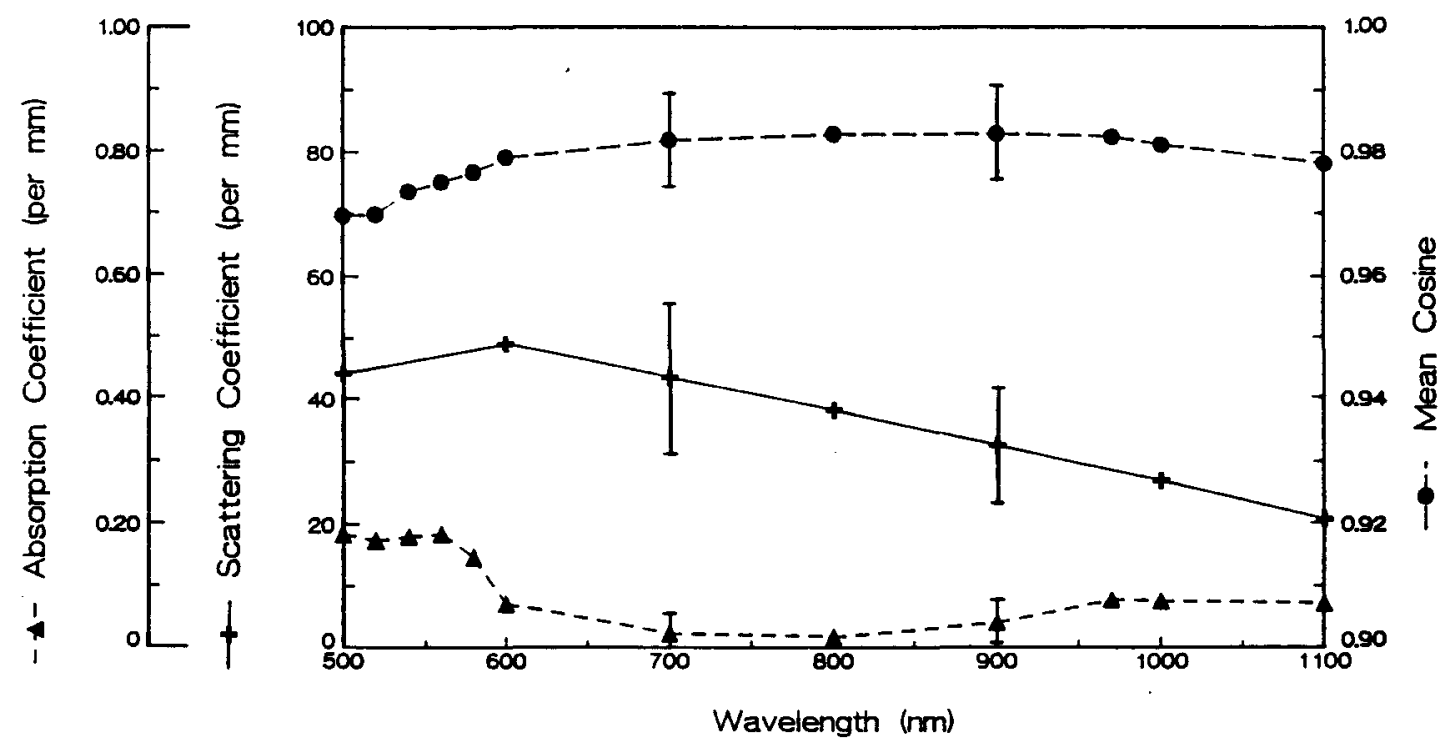

Figure 4.16 (continued)

c) fibrocystic disease

d) fibroadenoma 
e)

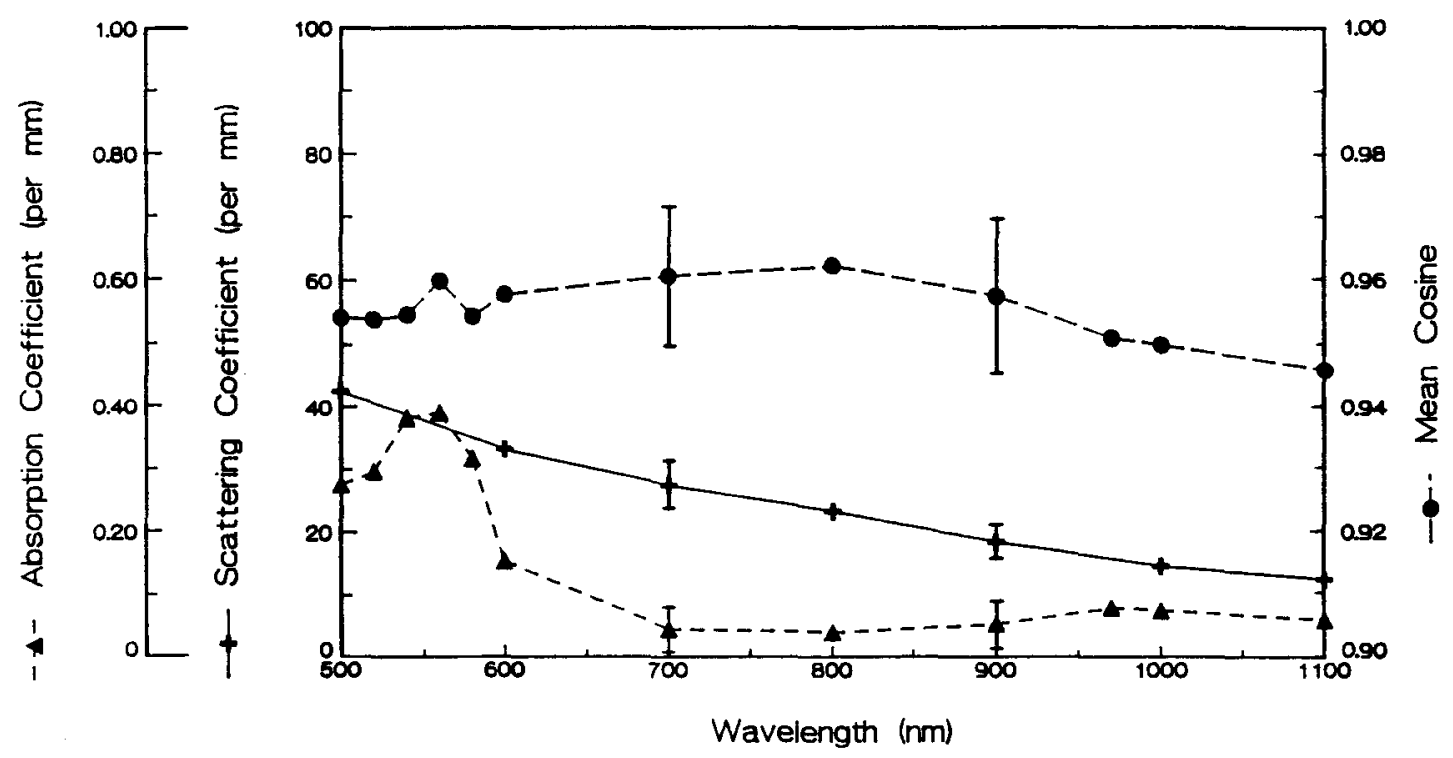

Eigure 4.16 (continued)

e) carcinoma 
All samples show significant absorption at wavelengths below $600 \mathrm{~nm}$. In this region, carcinoma shows the greatest absorption, with a coefficient as high as 0.38 $\mathrm{mm}^{-1}$. The absorption coefficient for fibrocystic disease is lower by a factor as great as eight. For the other samples, the absorption coefficients lie between these extremes. The differences are assumed to arise from variations in the blood content of the tissues.

At wavelengths greater than $600 \mathrm{~nm}$, the absorption coefficients of these samples do not demonstrate any significant differences. Within the uncertainties, the coefficients are similar to those derived for both Intralipid and the polystyrene spheres.

Scattering coefficients exceed the absorption coefficients by at least two orders of magnitude. In general, the scattering coefficients fall as the wavelength increases. The coefficients range between $30 \mathrm{~mm}^{-1}$ and 90 $\mathrm{mm}^{-1}$ at $500 \mathrm{~nm}$ and fall to within $10 \mathrm{~mm}^{-1}$ and $50 \mathrm{~mm}^{-1}$ at $1100 \mathrm{~nm}$. Adipose tissue appears to be an exception, in that the scattering remains constant as a function of wavelength at $30 \mathrm{t} /-6 \mathrm{~mm}^{-1}$. This result is consistent with the fact that the reflectance and transmittance, of all the adipose samples examined, change little with wavelength (figure $4.14)$. 
Although the scattering coefficients differ for each pathology, these differences are not statistically significant. Greater precision could perhaps be achieved by examining larger numbers of specimens.

The mean cosine of the scattering angle remains constant within $+/-0.01$ for all wavelengths for any given sample. The extreme values of this quantity are 0.945 and 0.985 , with no significant difference between samples. These high values are not unexpected since Flock et al (1987) reported mean scattering cosines of 0.941 for bovine muscle and 0.965 for white chicken muscle. However, their values of 0.771 for pig adipose and 0.639 for $v X-2$ tumour differ significantly from our results for fatty breast tissues and breast carcinoma.

4.3.5 Analysis using similarity relationships

A unique solution for the optical properties using the Monte Carlo method depends upon the measurement of $R_{\mu}$ ' $T_{M}$, and $\Sigma_{+}$. It is desirable to extract some information about the optical properties of those samples for which total attenuation data are not available. Van de Hulst (1980) has suggested that similar light distributions will be observed for differing materials provided that the absorption coefficient and the transport coefficient are matched where the transport coefficient is defined as the product of the scattering coefficient with $(1.0-9)$. 


$$
\begin{aligned}
& \text { That is } \quad \Sigma_{a_{1}}=\Sigma_{a_{2}} \quad 4.1 \\
& \text { and } \Sigma_{s_{1}} \cdot\left(1-g_{1}\right)=\Sigma_{s_{2}} \cdot\left(1-g_{2}\right) \quad 4.2
\end{aligned}
$$

where the subscripts refer to the material.

These relationships can be tested for our geometry using the Monte Carlo results of figure 2.4. For fixed values of $R_{m}$ and $T_{M}$, the optical properties have been derived for a range of $\Sigma_{+}$. The input values were typical of those measured for tissue. The results are presented in figure 4.17. The derived absorption coefficient is constant with $\Sigma_{f^{\prime}}$ within a standard deviation of $+/-0.016 \mathrm{~mm}^{-1}$. This error is smaller than that obtained for the tissue samples. The transport coefficient is also invariant with $\Sigma_{+}$within $+/-8$ percent. This difference is much smaller than the error determined for either the scattering coefficient or the value of $(1-g)$ for tissue.

We have applied the concept of similarity in the analysis of our experimental data. For each tissue sample, the total attenuation coefficient was taken to be equal to the value measured for the single specimen of that tissue type, as shown in figure 4.16. The optical properties were derived for each of the 33 samples at wavelengths of 540 , 700 , and $900 \mathrm{~nm}$. The data are presented in figure 4.18 as plots of the transport coefficient versus the absorption coefficient. 
a)

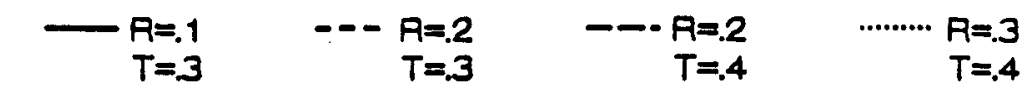

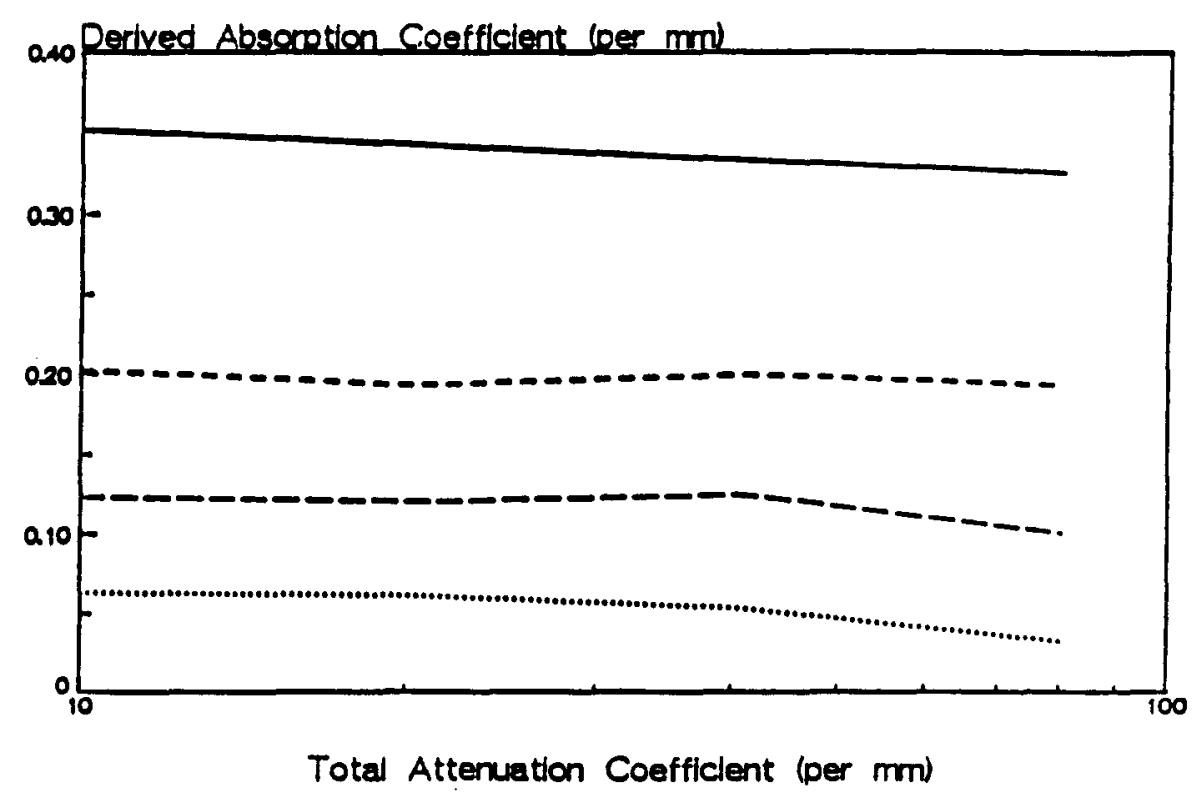

b)

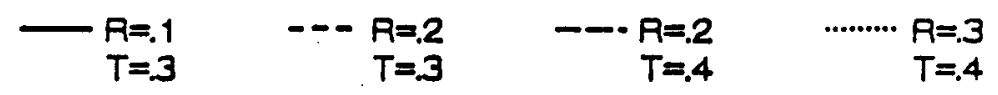

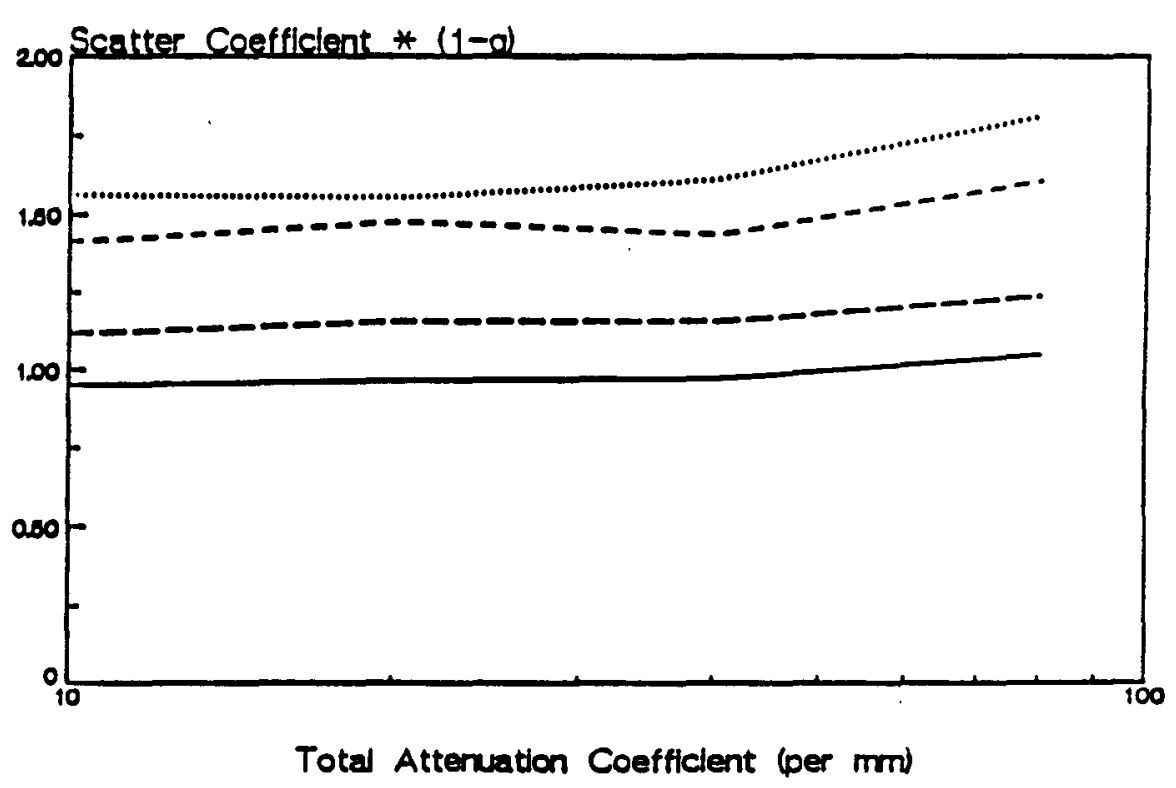

Figure 4.17 Plots of a) absorption coefficients

b) transport coefficients

, derived for selected values of $R$ and $T$, as functions of the total attenuation coefficient. 

+ gland- $\Delta$ adipose $\circ$ fibro- + fibroa- $\Delta$ carci- ular cystic denoma noma

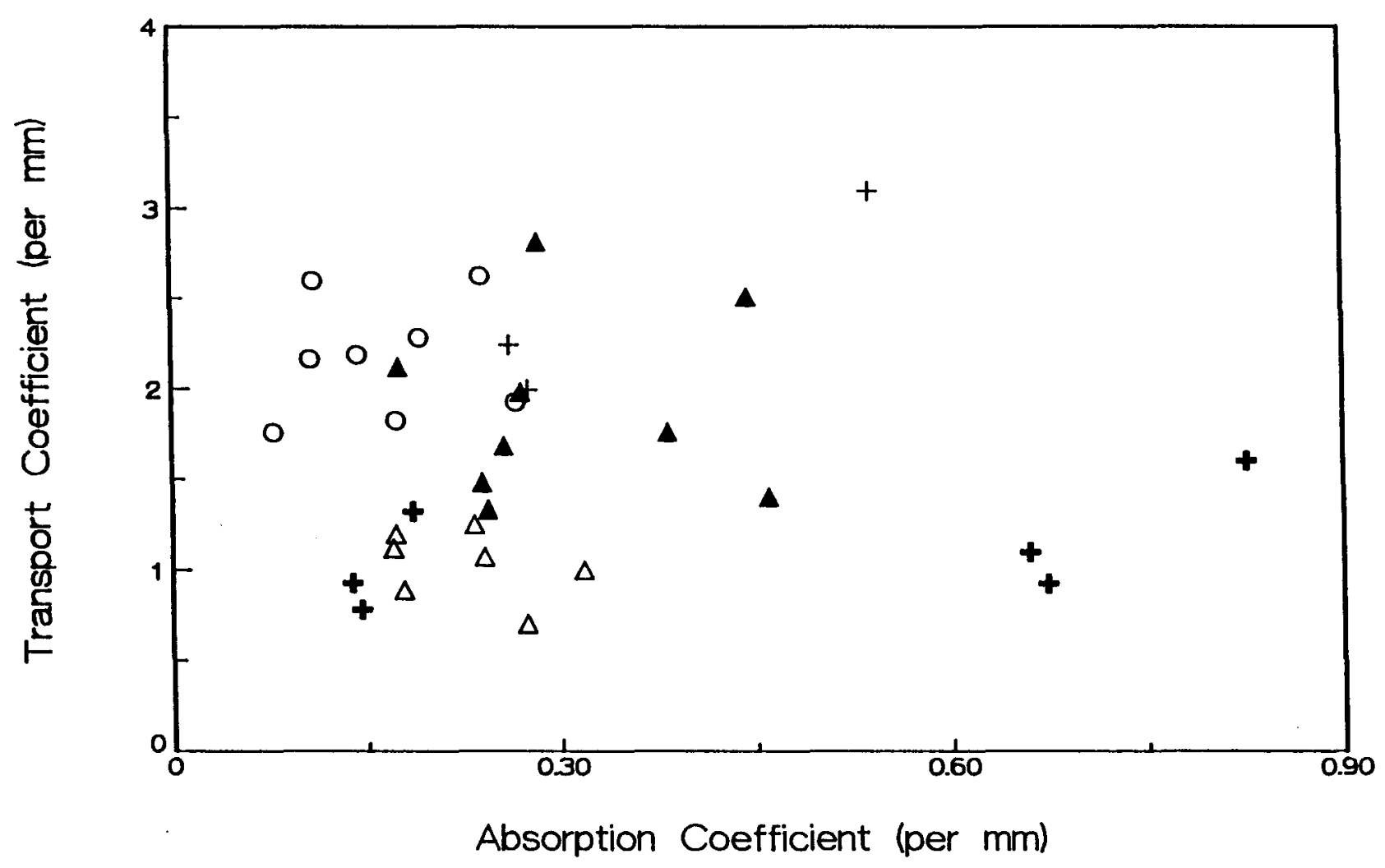

Figure 4.18 The transport coefficient plotted as a function of the absorption coefficient for each of 33 samples, a) at wavelength $=540 \mathrm{~nm}$. Both quantities were derived assuming similarity (i.e. invariance with $\Sigma_{+}$). 

+ gland- $\Delta$ adipose $\circ$ fibro- + fibroa- $\triangle$ carci- ular cystic denoma noma

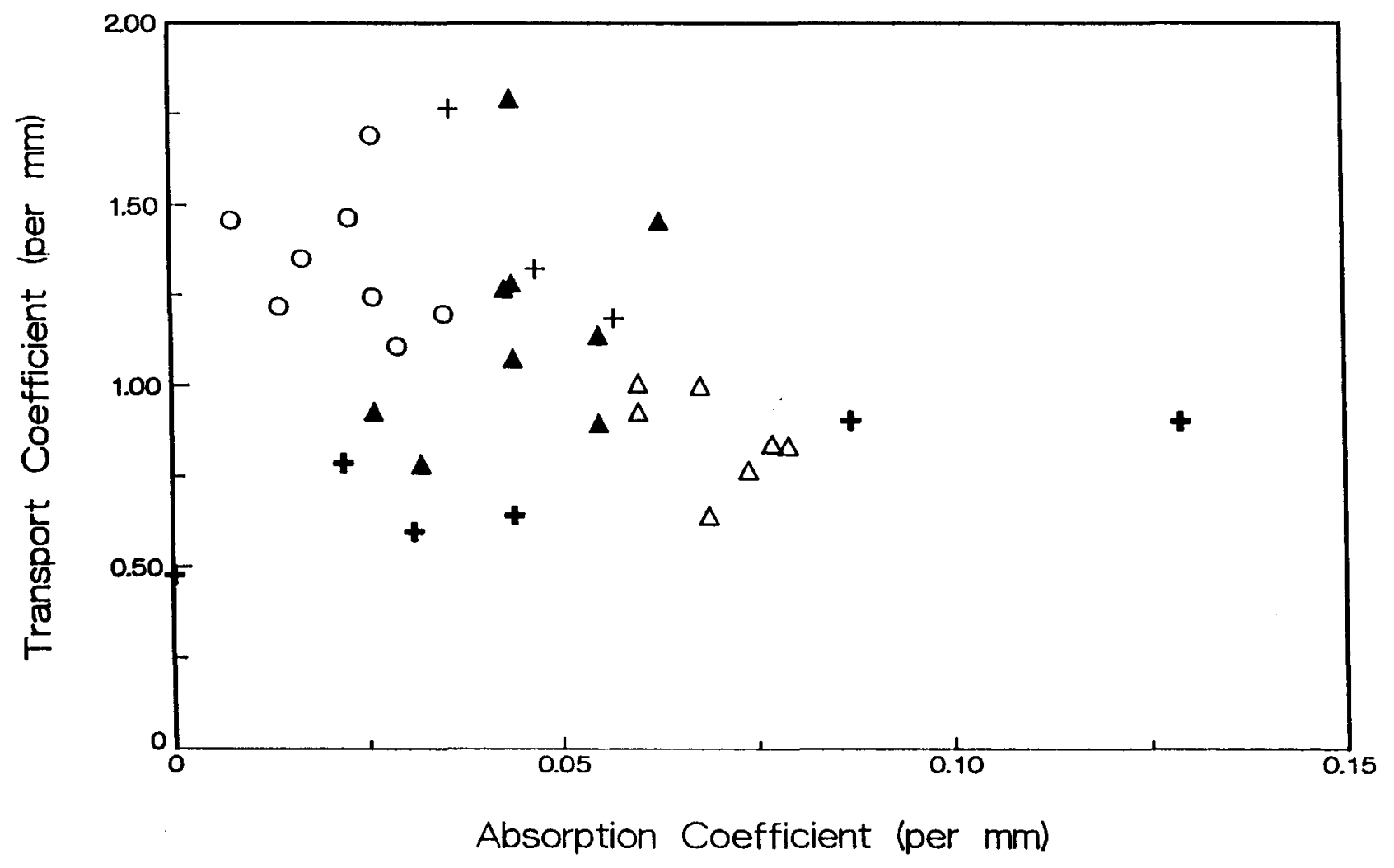

Figure 4.18 (continued) b) wavelength $=700 \mathrm{~nm}$. 


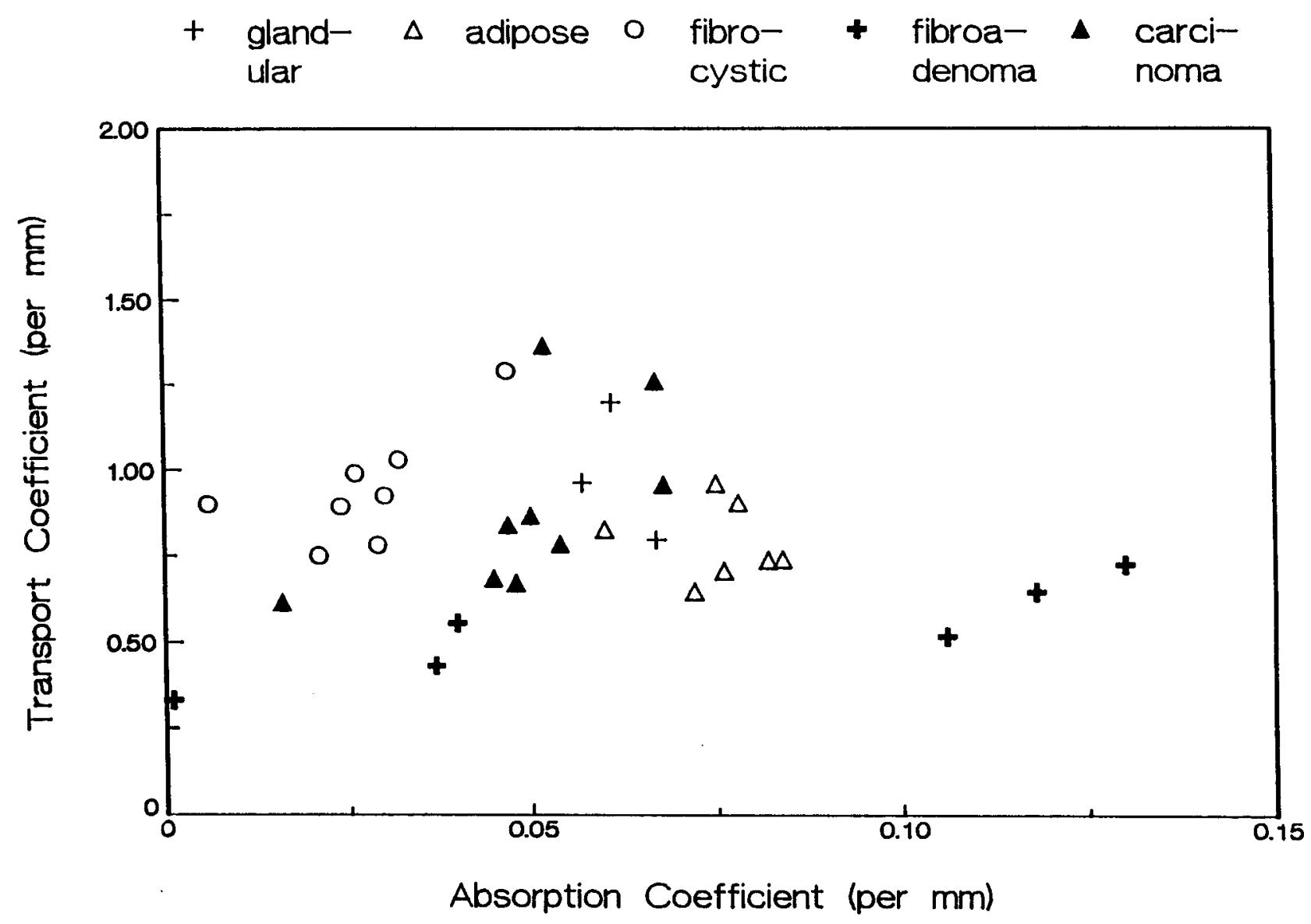

Figure 4.18 (continued) c) wavelength $=900 \mathrm{~nm}$. 
Clustering of the data points for each tissue type is evident at each wavelength. The points for fibrocystic disease and adipose tissue are particularly well clustered and separated from each other at all three wavelengths. This separation is mainly a function of the transport coefficient at $540 \mathrm{~nm}$, differs along both axes at $700 \mathrm{~nm}$, and is mainly a result of absorption differences at $900 \mathrm{~nm}$. The transport coefficient for fibrocystic disease falls with increasing wavelength, while that for adipose tissue remains unchanged.

The data points representing carcinoma generally fall between the clusters for fibrocystic disease and adipose tissue with little overlap. Normal glandular tissue is similar to carcinoma at each wavelength.

Fibroadenoma exhibits a large range of absorption coefficients at all wavelengths; however, the range of transport coefficients is small. The variation in absorption coefficients may indicate a greater variability in blood content than other tissues. At $900 \mathrm{~nm}$, all tissue types, except fibroadenoma, are differentiated mainly in terms of their absorption coefficients. 


\subsubsection{Phantom materials for breast tissues}

Quantitative evaluation of transillumination

devices and techniques can only be accomplished through use of appropriate phantom materials.

our results indicate that Intralipid is not an ideal material, since the mean scattering angle is considerably larger than that for soft tissue. Similarity relationships can be applied (Hansen, 1988) in order to scale the optical properties; however, the validity of this approach may depend on the particular problem being investigated. Polystyrene microspheres provide better agreement with tissue and have the advantage that the mean scattering angle can be selected through the choice of sphere size. Scattering coefficients can be matched by changing the concentration of spheres in the suspension. Modeling the spectral response of tissues requires use of a material to mimic the absorption by hemoglobin. Satisfactory substitutes have not yet been developed. The use of whole blood is still recommended (Linford et al, 1987) despite problems associated with decomposition. 


\subsection{Future work}

The major limitation of the work described is that a complete derivation of the optical properties was only performed for one sample for each pathology. In order to more accurately define the normal range of these properties for each pathology, it will be necessary to examine the total attenuation coefficient of a larger number of samples.

4.4.1 Computer simulation of transillumination

The fundamental issue which must be addressed following the completion of the above work is the effect of the optical properties of different breast tissues on transillumination imaging. Using a computer model, we will attempt to optimize the imaging parameters. For this purpose, a Monte Carlo technique has been developed which simulates light transport in optically thick media.

The imaging process will be evaluated for a variety of incident spectra, using realistic tissue geometries. The ideal imaging wavelength will depend on

1) the total attenuation of light in the breast,

2) the effect of differential attenuation by various tissue types on contrast,

and 3) the effect of scatter on image quality. 
For visible wavelengths, absorption by blood will greatly reduce the transmission of light through the breast. Use of longer wavelengths will permit greater penetration of light through tissues.

The optical properties of fat appear to be invariant over the range of wavelengths from 700 to $900 \mathrm{~nm}$, while those of other tissues change. Subtraction imaging should, therefore, be able to distinguish between fat and other breast tissues. The optimal wavelengths may depend on the pathology of the tissues examined.

\subsubsection{Development of equipment}

Application of these investigations requires the development of specifications for clinical diaphanography systems. We must address practical problems including the selection of light sources, detectors and display systems. Better phantom materials must also be developed, to allow objective evaluation of system performance.

Ultimately, this work should lead to clinical tests to establish the value of transillumination in breast screening and diagnosis. 


\subsubsection{Advanced techniques}

Two methods, which may hold promise for future diaphanography systems, are:

1. cross-sectional imaging,

and

2. time of flight detection.

Computerized reconstruction of cross-sectional

images is a widely used diagnostic technique (e.g.

computerized tomography, magnetic resonance imaging), which has the advantage of providing more precise localization of tissues. Jackson et al (1987) concludes that this technique has the potential to improve the sensitivity of transillumination. However, since the images are formed by scattered photons rather than by unattenuated photons, appropriate reconstruction algorithms are not easily developed. Clinical equipment is not yet available.

Time of flight detectors may be able to improve the resolution of transillumination images by discriminating against scattered photons (Maarek et al, 1986). Since light travels at a finite velocity, scattered light will take a longer time to reach the detector than unattenuated photons. A time gate on the detector could select photons which traverse the material along a more direct path. Light sources required for this technique must emit very short pulses of high intensity light. The availability of picosecond lasers will, undoubtedly, lead to further work in this area. 
CHAPTER 5

CONCLUSIONS

A technique has been developed to determine the absorption and scattering coefficients of tissues for light at wavelengths between 500 and $1100 \mathrm{~nm}$. In addition, the mean cosine of the scattering angle is determined assuming that the scatter phase function can be represented by a Henyey-Greenstein function. The method is based on a combination of experimental data and a Monte Carlo model of light transport. The input parameters required for the simulation include measured values of the diffuse reflectance and transmittance of prepared samples as well as the total attenuation coefficient. The validity of the technique was established by experiments with suspensions of polystyrene microspheres and ink having known scattering and absorption properties.

This method has been applied to the investigation of diseased and normal breast tissues. The scattering coefficients were found to 1 ie in the range $30 \mathrm{~mm}^{-1}$ to $90 \mathrm{~mm}^{-1}$ at $500 \mathrm{~nm}$, and fall smoothly with increasing wavelength to within $10 \mathrm{~mm}^{-1}$ and $50 \mathrm{~mm}^{-1}$ at $1100 \mathrm{~nm}$. 
The scattering coefficient for adipose tissue differs from that of other tissues, remaining constant with wavelength at $30+/-6 \mathrm{~mm}^{-1}$. The mean cosine of the scattering angle for all tissue types examined lies in the range 0.945 to 0.985 , and appears to be invariant with wavelength. scattering is the predominant attenuation process in tissues, with coefficients that exceed the absorption coefficients by greater than two orders of magnitude. The absorption coefficients are strongly affected by the presence of blood, particularly at wavelengths below 600 $\mathrm{nm}$.

Tissues of various pathologies have differing absorption coefficients and scattering properties. These differences are wavelength dependent and cannot be attributed solely to differences in blood concentration. The implications for transillumination imaging have yet to be determined. Although the optical properties of carcinoma resemble those of normal glandular tissue, it may be possible to differentiate malignancies based on the distortion of normal structures if these can be visualized. 


\section{APPENDIX A}

\section{PROGRAM FOR CONTROL OF THE EXPERIMENT}

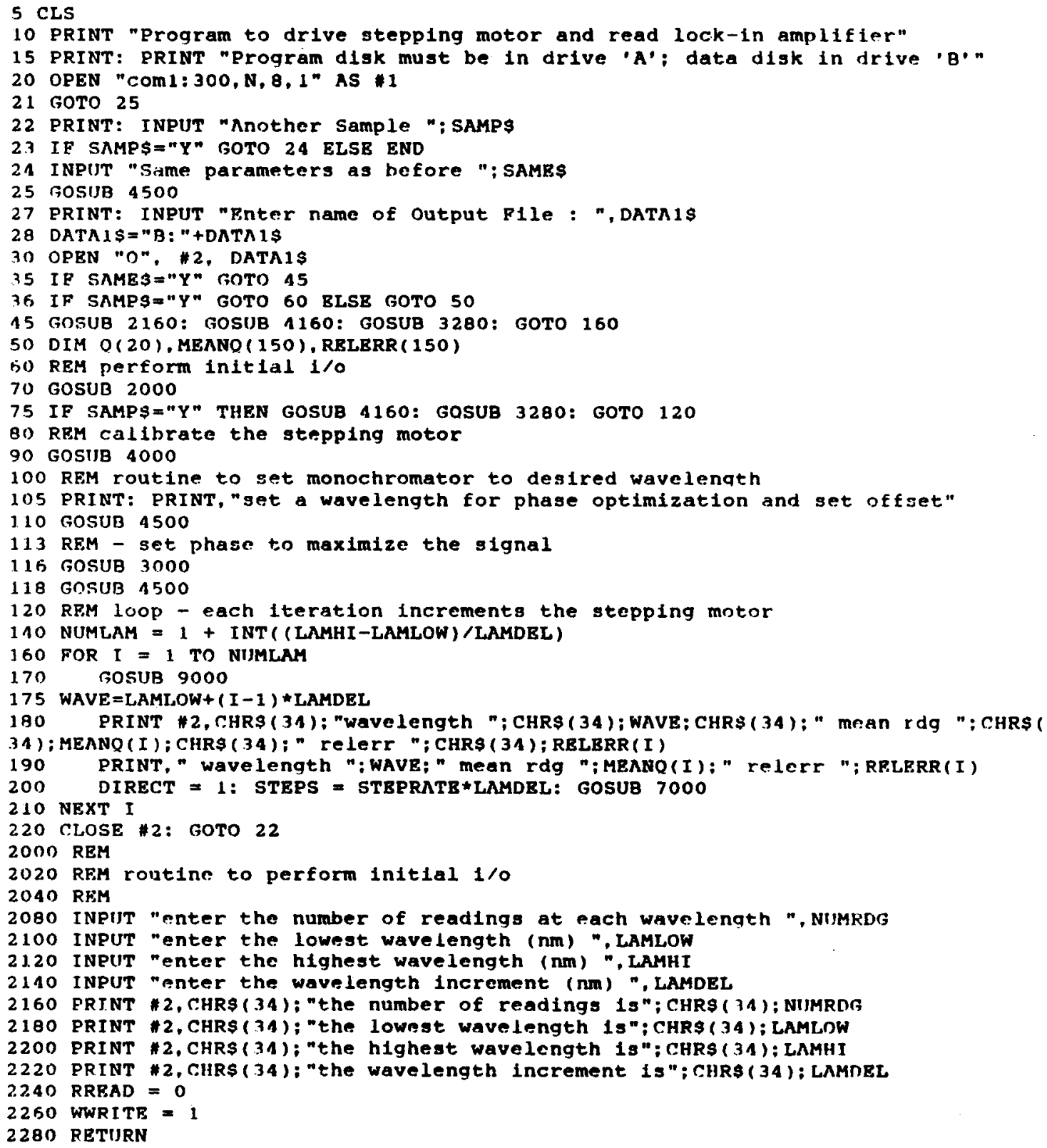




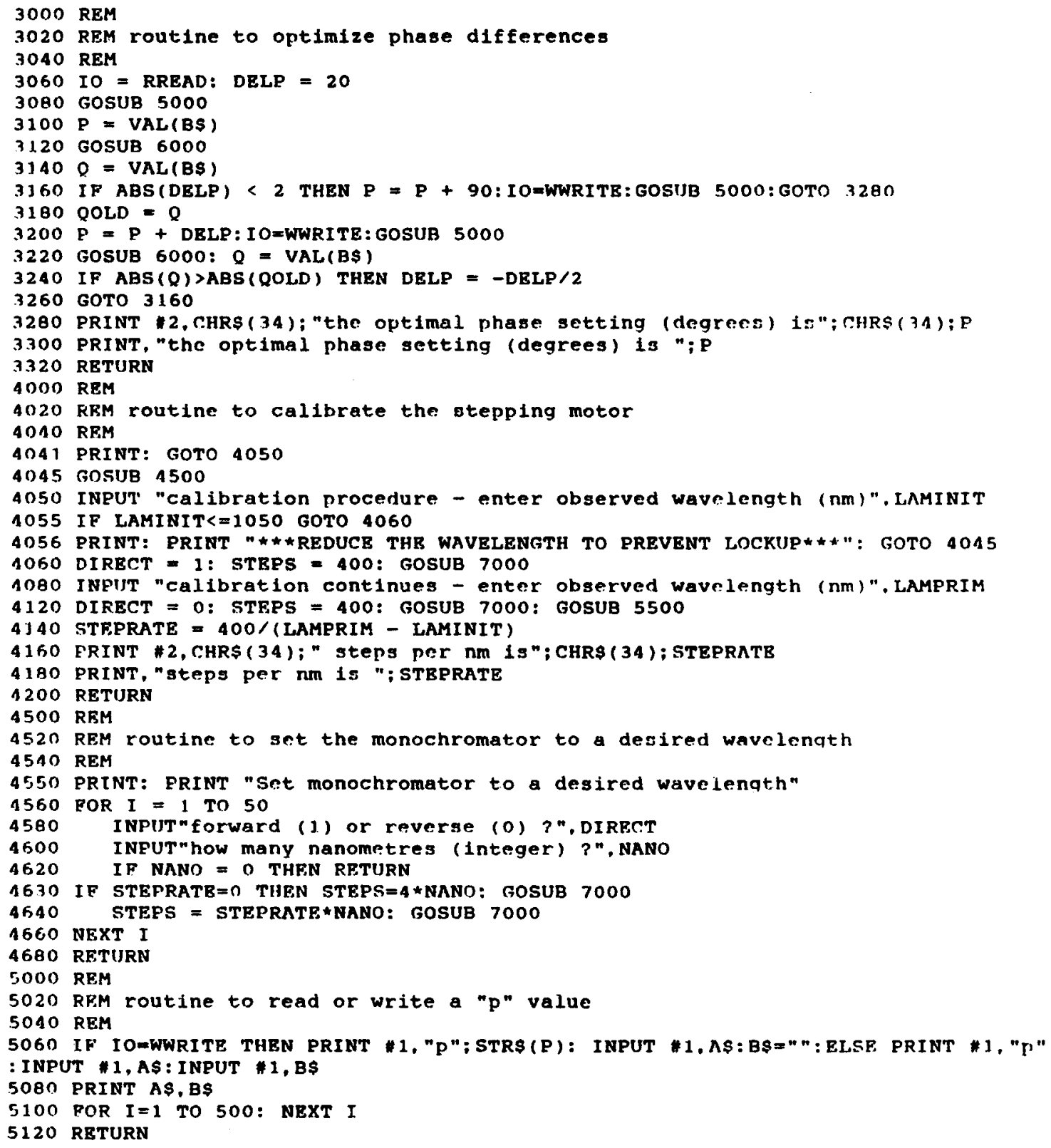




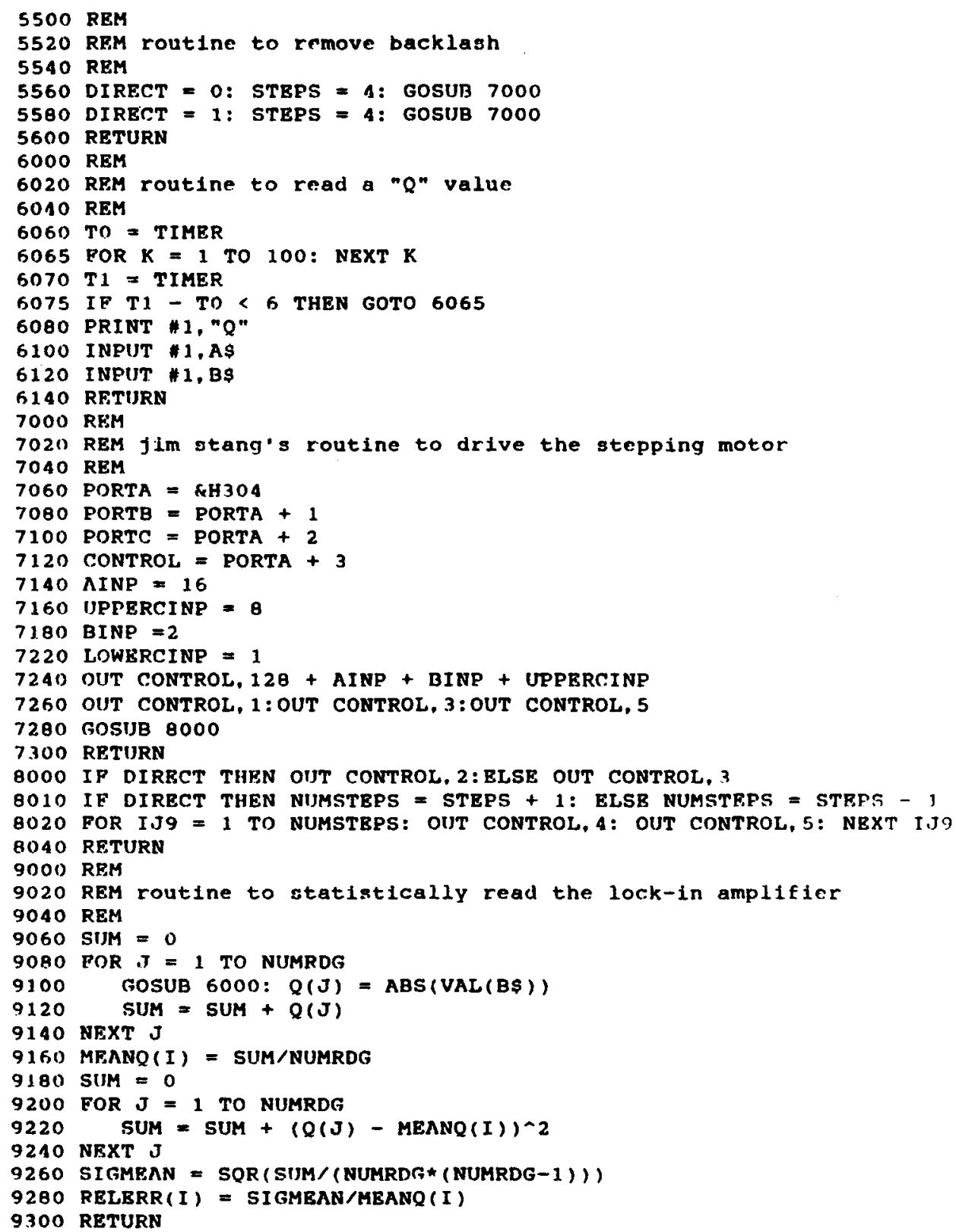




\section{APPENDIX B}

\section{DERIVATION OF REFLECTANCE AND TRANSMITTANCE FORMULAE}

An experimental technique using an integrating sphere has been described in chapter 3 for measuring quantities which are related to the actual reflectance and transmittance of a sample.

Refering to figure $3.6(a)$, we will first derive the relationship for reflectance measurements. The light flux entering the sphere is given by $\phi_{0}$. On striking the sample, the flux reflected back into the sphere is given by

$$
R \phi_{0}
$$

B. 1

where $R$ is the sample reflectance. This light, which is directly reflected by the sample, is not detected by the photodiode, due to the presence of the light baffle.

We will assume that a negligible amount of the scattered light is lost through the entrance port of the sphere. In this case, the flux remaining in the sphere after all photons have been scattered once on the inner sphere surface is given by

$$
\phi_{1}=R_{s} R \phi_{0}
$$

where $R_{s}$ is the reflectivity of the sphere coating. 
Since the sphere coating acts as a diffuse reflector, we assume that the light is, now, uniformly distributed throughout the sphere. The detector signal arising from this first order scatter is given by

$$
I_{1}=C R_{5} R \phi_{0}\left(A_{0} / A_{+}\right) \quad \text { B.3 }
$$

where $A_{0} / A_{+}$is the ratio of detector area to the total inner surface area of the sphere, and $C$ is a calibration factor for the detector response.

The flux remaining in the sphere, after all second order reflections on the sphere wall, is given by

$$
\phi_{2}=R_{5}^{2} R \phi_{0} \quad \text { B. } 4
$$

The losses due to the presence of the detector, sample and beam entrance ports are assumed to be negligible in comparison with the losses in the sphere coating, despite the high reflectivity of the coating. This results from the fact that the inner sphere area is much greater than the combined areas of the ports.

The detected signal from the second order flux is

$$
I_{2}=C R_{s}^{2} R \phi_{0}\left(A_{0} / A_{+}\right) \quad \text { B.5 }
$$

Similarly, the flux remaining in the sphere after all third order reflections is given by

$$
\phi_{3}=R_{s}^{3} R \phi_{0} \quad \text { B.6 }
$$

and the detected signal arising from this flux is

$$
I_{3}=C R_{s}^{3} R \phi_{0}\left(A_{p} / A_{+}\right) \quad \text { B. }
$$


The total detected signal is the sum of all orders of scatter contribution

$$
\begin{aligned}
I_{R} & =I_{1}+I_{2}+I_{3}+\ldots \\
& =C R_{s} R \phi_{0}\left(A_{0} / A_{+}\right)\left(1+R_{s}+R_{s}^{2}+\ldots\right) \\
& =C R_{s} R \phi_{0}\left(A_{D} / A_{+}\right) /\left(1-R_{s}\right)
\end{aligned}
$$

If the sample is replaced with a barium sulfate reflection plate having the same reflectance as the sphere coating, then the detected signal is given by

$$
I_{s}=C R_{s}^{2} \phi_{0}\left(A_{b} / A_{+}\right) /\left(1-R_{s}\right)
$$

Taking the ratio of the reading with the sample to the reading with the reflectance plate, we obtain

$$
I_{R} / I_{s}=R / R_{S} \quad \text { B.10 }
$$

The sample reflectance can, therefore, be determined from

$$
R=R_{s}\left(I_{R} / I_{s}\right) \quad \text { B.11 }
$$

For the case of a sample held between glass plates, the reflectance determined in this way will not equal the true reflectance of the sample; rather, it will correspond to the reflectance as defined for the Monte Carlo calculations in section 2.3.3. Under this definition, the reflectivity is modified by specular reflection losses, absorption in glass and geometric boundary conditions. 
We will next derive the relationship for transmittance, based on the measurement geometry shown in figure $3.6(b)$. Since the sphere has been rotated by 180 degrees from the reflectance geometry, the light flux incident on the sample is $\phi_{0}$, as for the reflectance measurements. The flux transmitted by the sample is

$$
T \phi_{0} \quad \text { B. } 12
$$

where $T$ is the sample transmittance (as defined in section 2.3.3 for a sample between glass slides). None of the directly transmitted light is detected by the photodiode, due to the presence of the light baffle. The flux remaining after all photons have been scattered once on the inner sphere surface is given by

$$
\phi_{1}=R_{s} T \phi_{0}
$$

where $R_{s}$ is the reflectivity of the sphere coating. From this point, the detection of the light is developed in the same manner as for the reflectance measurements. The resulting formula for determining the sample transmittance is

$$
T=R_{s}\left(I_{T} / I_{s}\right)
$$

where $I_{\boldsymbol{T}}$ is the detector signal obtained with the sample in the transmittance geometry and, $I_{s}$ is the detector signal for the barium sulfate plate obtained using the reflectance geometry as before. 
The validity of the formulae which have been developed depends upon the assumption that light losses at the detector, sample and beam ports are negligible with respect to losses resulting from interactions with the sphere coating. A detailed geometrical analysis, not presented here, was conducted using the actual dimensions of our sphere. In no case do these corrections result in a change of greater than 0.03 in the determined values of the reflectance or transmittance. Further verification of this approach, using reflectance standards, is provided in section 3.3 of the text. 


\section{BIBL IOGRAPHY}

Bevington, P.R., 1969 Data Reduction and Error Analysis for the Physical Sciences, McGraw-Hill Book Co., New York.

Bohren, C.F., and D.R.Huffman, 1983 Absorption and Scattering of Light by Small particles, John wiley and Sons Inc., New York.

Bolin, F.P., L.E.Preuss, R.C.Taylor and R.J.Ference, 1989 "Refractive index of some mammalian tissues using a fiber optic cladding method", Applied optics 28 p. 2297-2303.

Byrd, B.F., 1980 "ACS/NCI breast cancer detection demonstration projects", Cancer 46 p.1084-1086.

Carlsen, E., 1982 "Transillumination light scanning", Diagnostic Imaging $\underline{4}$ p. 28-33.

Carter, L.L., and E.D.Cashwell, 1975 Particle-Transport Simulation with the Monte Carlo Method, Technical Information Center, Office of Public Affairs, us Energy Research and Development Administration.

Clarke, E.A., N.Kreiger and L.D.Marrett, 1987 "Cancer incidence, mortality and treatment in ontario", p.179-198 in Cancer in Ontario, Ontario Cancer Treatment and Research Foundation, Toronto, Ontario.

CRC Handbook of Chemistry and Physics, 1983-1984 64th edition, CRC Press Inc., Boca Raton, Florida.

Crilly, R., 1986 "A study of the optical properties of soft tissue in the near infra-red", Med.Phys. 13 p.603.

Cutler, M., 1929 "Transillumination as an aid in the diagnosis of breast lesions", Surgery, Gynecology and Obstetrics 48 p.721-729.

Day, N.E., and A.B.Miller, 1988 Screening for Breast Cancer, Hans Huber Publishers, Toronto, Ontario.

Driscoll, W.G., and W.Vaughan, 1978 Handbook of Optics, McGraw-Hill Book Co., New York.

Duderstadt, J.J., and L.J.Hamilton, 1976 Nuclear Reactor Analysis, John Wiley and Sons Inc., New York. 


\section{Bibliography (continued)}

Egan, R.L., 1972 Mammography, C.C.Thomas Publisher, Springfield, Illinois.

Ertefai, S., and A.E.Profio, 1985 "Spectral transmittance and contrast in breast diaphanography", Med.Phys. 12 p. 393-400.

Fagerberg, G., L.Baldetorp, O.Grontoft, B.Lundstrum, J.C.Manson and B.Nordenskjold, 1985 "Effects of repeated mammographic screening on breast cancer stage distribution", Acta Radiologica Oncology 24 p.465-473.

Flock, S.T., B.C.Wilson and M.S.Patterson, 1987 "Total attenuation coefficients and scattering phase functions of tissues and phantom materials at $633 \mathrm{~nm} "$, Med.Phys. 14 p.835-841.

Hansen, V.N., 1988 "Breast transillumination - experimental and computer models", Masters thesis, McMaster University.

Henderson, I.C., and G.P.Canellos, 1980 "Cancer of the breast: the past decade", New England Journal of Medicine 302 p.17-30,78-90.

van de Hulst, H.C., 1980 Multiple Light Scattering Tables, Formulas, and Applications, Academic Press Inc., New York.

Isard, H.J., 1984 "Other imaging techniques", Cancer $\underline{53}$ p. 658-664.

Jackson, P.C., P.H.Stevens, J.H.Smith, D.Kear, H.Key and P.N.T.Wells, 1987 "The development of a system for transillumination computed tomography", British Journal of Radiology 60 p.375-380.

Johns, P.C., and M.J.Yaffe, 1987 "X-ray characterisation of normal and neoplastic breast tissues", Physics in Medicine and Biology 32 p.675-695.

Jones, C.H., 1982, "Methods of breast imaging", Physics in Medicine and Biology 27 p.463-499.

Keys, H.M., R.F.Bakemeier and E.D.Savlov, 1983 "Breast cancer" p.120-140 in Clinical Oncology for Medical Students and Physicians - A Multidisciplinary Approach, American Cancer Society. 
Bibliography (continued)

Kortum, G., 1969 Reflectance Spectroscopy - Principles, Methods, Applications, Springer-Verlag Inc., New York.

Linford, J., S.Shalev, J.Bews, R.Brown and H.Schipper, 1986 "Development of a tissue-equivalent phantom for diaphanography", Med.Phys. 13 p.869-875.

Maarek, J.M., G.Jarry, J.Crowe, M.A.Deugnier, Bui-Mong-Hung and D. Laurent, 1986 "Simulation of laser tomoscopy in a heterogeneous biological medium" Med. \& Biol.Eng. \& Comp. 24 p.407-414.

Marchesini, R., A.Bertoni, S.Andreola, E.Melloni and A.E.Sichirollo, 1989 "Extinction and absorption coefficients and scattering phase functions of human tissues in vitro" submitted to Applied optics.

NCRP Report No. 85, 1986 Mammography - A Users Guide, National Council on Radiation Protection and Measurements, Bethesda, Maryland.

Ohlsson, B., J.Gundersen and D.Nilsson, 1980

"Diaphanography: a method for evaluation of the female breast", World Journal of Surgery 4 p.701-707.

Profio, A.E., G.A.Navarro and O.W.Sartorius, 1988 "Scientific basis of breast diaphanography", Med.Phys. 16 p.60-65.

Shapiro, S., W.Venet, P.Strax, L.Venet and R.Roeser, 1982 "Ten-to-fourteen-year effect of screening on breast cancer mortality", Journal of the National Cancer Institute 69 p.349-355.

Tabar, L., A.Gad, L.H.Holmberg, U.Ljungquist, C.J.G.Fagerberg, L.Balde torp, O.Grontoft, B. Lundstrum, J.C.Manson, G.Eklund, N.E.Day and F.Pettersson, 1985 "Reduction in mortality from breast cancer after mass screening with mammography", The Lancet I(1985) p. 829-832.

Wallberg, H., 1985 "Diaphanography in various breast disorders", Acta Radiologica Diagnosis 26 p.271-276.

Watmough, D.J., 1982 "Diaphanography, mechanism responsible for the images", Acta Radiologica Oncology 21 p.11-15. 
Bibliography (continued)

Wilksch, P.A., F.Jacka and A.J.Blake, 1984 "Studies of light propagation through tissue", p.149-161 in Porphyrin Localization and Treatment of Tumours, Alan R. Liss Inc., New York.

Wilson, B.C., and G.Adam, 1983 "A Monte Carlo model for the absorption and flux distributions of light in tissue", Med.Phys. 10 p.824-830.

Wilson, B.C., and M.S.Patterson, 1986 "The physics of photodynamic therapy", Physics in Medicine and Biology 31 p. 327-360.

Wilson, B.C., M.S.Patterson and D.M.Burns, 1986 "Effect of photosensitizer concentration in tissue on the penetration depth of photoactivating light", Lasers in Medical Science 1 p.235-244.

Wolfe, J.N., 1972 Xeroradiography of the Breast, c.C.Thomas Publisher, Springfield, Illinois.

Wyman, D.R., and M.S.Patterson, 1988 "A discrete method for anisotropic angular sampling in Monte Carlo simulations", J.Comp.Phys. 76 p.414-424.

Wyman, D.R., M.S.Patterson and B.C.Wilson, 1989

"Similarity relations for anisotropic scattering in Monte Carlo simulations of deeply penetrating neutral particles", J.Comp.Phys. 81 p.137-150. 